\title{
CINEMA ACROSS BORDERS : NATIONAL DIFFERENCES IN SÁMI FILMMAKING IN THE NORDIC COUNTRIES
}

\author{
by Rachael Crawley \\ Bachelor of Arts, Cinema Studies/Russian Language and Literature \\ University of Toronto \\ Toronto, Ontario \\ 2013

\begin{abstract}
A thesis presented to Ryerson University
in partial fulfillment of the degree of Master of Arts

in Film + Photography Preservation and Collections Management
\end{abstract} \\ Toronto, Ontario, Canada, 2017 \\ (C) Rachael Crawley, 2017
}




\section{Author's Declaration}

I hereby declare that I am the sole author of this thesis. This is a true copy of the thesis, including any required final revisions, as accepted by my examiners.

I authorize Ryerson University to lend this thesis to other institutions or individuals for the purpose of scholarly research.

I further authorize Ryerson University to reproduce this thesis by photocopying or by other means, in total or in part, at the request of other institutions or individuals for the purpose of scholarly research.

I understand that my thesis may be made electronically available to the public. 


\begin{abstract}
Cinema Across Borders: National Differences in Sámi Filmmaking in the Nordic Countries
\end{abstract}

Master of Arts, 2017

Rachael Crawley

Film + Photography Preservation and Collections Management

Ryerson University

The cinema of the Sámi people, of northern Fennoscandia and Russia (Sápmi), has flourished since the turn of the millennium. This thesis summarizes the history of Sámi film, its current infrastructure, and the differences in filmmaking trends between the three Nordic countries of Sápmi. It also includes a list of all known Sámi productions and organizations funding Sámi film. By exploring the differences in funding in the Nordic countries, it examines the relative lack of Sámi film production and infrastructure in Sweden, as compared to Norway and Finland. The paper compares the development of the film industry, in each country of Sápmi, with the political history and current legal status of the Sámi, finding that the success of Sámi film is correlated with greater autonomy in government and language rights. 


\section{Acknowledgements}

I am grateful to Jan Olsson, my first reader, for his insight and guidance, and to Alicia Fletcher, my second reader, for her meticulous attention to this thesis, and her initial encouragement of studying Sámi film.

I would like to thank Jon Wengström, my supervisor at the Swedish Film Institute, and my colleagues in Stockholm for their patience and support. Thanks as well to the faculty and staff of the Film and Photography Preservation and Collections Management program for all that

they have taught me, and to my fellow students for their friendship, support, and ideas. I would also like to thank my Swedish teacher, Lilian Junkin, for her help in learning the language.

Thank you to Oskar Östergren from the Dellie Maa film festival and the International Sámi Film Institute, for speaking with me about Sámi film funding and the creation of Dellie Maa; and to Kaija Anttonen from the Skábmagovat Film Festival and the Sámi Film Database, for her insight into the composition of the database and the history of Skábmagovat.

For their unwavering belief in me, and for all they have done to support me, I thank Helen and LeRoy Worley, and David Crawley and Joan Bubbs. 


\section{Table of Contents}

Author's Declaration ................................................................................. ii

Abstract ............................................................................................... ii

Acknowledgements ............................................................................ iv

Table of Contents ....................................................................................

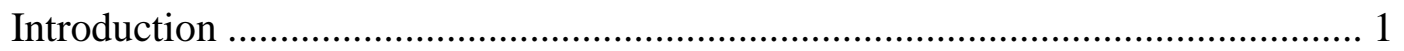

Chapter 1: The Sámi People ........................................................................... 2

Chapter 2: Literature Review .................................................................. 4

2.1 Films and Their Content ........................................................... 5

2.2 Sámi History and Political Status .................................................. 8

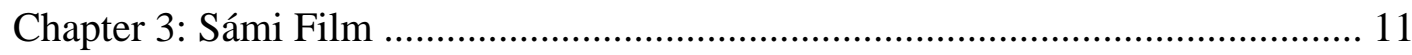

Chapter 4: Analysis of Sámi Films And Institutions ...................................... 14

Chapter 5: Sámi Rights by Country ............................................................... 20

Appendix A: Sámi History and Facts ...................................................... 27

Appendix B: Sámi Film History, Exhibition and Institutions ........................... 30

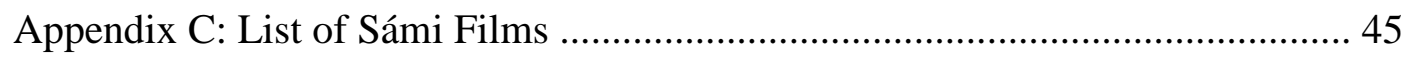

Appendix D: Sámi Films By the Numbers ................................................... 126

Bibliography .................................................................... 132 


\section{Introduction}

In the case of the Sámi people, the policies of four different countries - Norway, Sweden, Finland and Russia - create widely differing legal and cultural contexts across the region. This disparity is reflected in local film production, which varies both in number of films produced, and in development of filmmaking infrastructure, from country to country. In countries with a strong legal basis for political autonomy and recognition, such as Norway and Finland, there is greater development of institutional and financial support for filmmaking, as evidenced in the creation of the International Sámi Film Institute. Conversely, in Russia, which has little recognition of the Sámi, very few films have been made. Sweden has taken steps towards Sámi recognition, but still lags behind Norway and Finland in implementation linguistic and political support. It has produced many films from Sámi filmmakers, but does not possess the Sámifocused funding and educational organizations offered to filmmakers in Norway and Finland.

Current scholarship on Sámi film, especially in the English language, is strongly focused on analysis of individual films or the careers of particular filmmakers, with little attention paid to the industry itself (though some articles exist on Sámi radio and television broadcasting). Films can also be studied comparatively with Indigenous productions from other countries, notably Canada, the United States and Australia, though these analyses usually focus on film content. Though I have created a list of the known Sámi films from its inception in 1963, it is not from a textual perspective, but instead as an indicator of the industry's growth. By documenting the funding of films across Sápmi, and describing the presence and impact of local filmmaking institutions, this thesis explores the differences between the infrastructure of the Sámi film industry in the three Nordic countries of Sápmi -- Sweden, Norway and Finland -- and seeks to understand the political contexts in which each developed. 


\section{Chapter 1: The Sámi People}

The Sámi (or Saami) are the Indigenous people of the Sápmi region, consisting of northern Norway, Sweden, Finland, and the Kola Peninsula of Russia. The previous term for the area, "Lappland", is now considered to be outdated by the Sámi (though it is still used in some deesignations by national governments), as are the terms "Lapp" for the people and "Lappish" for the language. ${ }^{1}$ Historically, the Sámi have lived by fishing, gathering, trapping, and especially reindeer herding. Outside of Sápmi, there are about 30,000 people of Sámi descent in the United States, and significant populations in Ukraine and other ex-Soviet states. There are ten generally accepted extant Sámi languages, all from the Finno-Ugric language family -- making them close relatives of Finnish and Estonian -- which range in number from 25,000 speakers to two (as well as several extinct variants) $)^{2}$. There are about 70-80,000 Sámi people in the world, including 40,000 in Norway, 20,000 in Sweden, and 8,000 in Finland ${ }^{3}$.

Sámi history in the countries of Sápmi has been marked by marginalization, including collectivization and forced deportation to Lovozero, Murmansk Oblast in Soviet Russia ${ }^{4}$, denial of language rights, forced conversion to Lutheran or Orthodox Christianity, "Norwegianization" campaigns ${ }^{5}$, and mandatory (and often inadequate) education in "Sámi boarding schools" in the Nordic countries ${ }^{6}$. This process has recently been depicted in the Swedish film Sameblod (Amanda Kernell, 2017). Current issues include conflict over land rights, which threaten the

\footnotetext{
${ }^{1}$ Frawley, William J., International Dictionary of Linguistics: AAVE-Esperanto, Vol. 1, 2nd Edition, s.v. "Sàmi, Inari", New York: Oxford University Press, 2003, 332.

2 "Uralic", Ethnologue.com, accessed 9 May 2017, https://www.ethnologue.com/subgroups/uralic.

3 "The Sámi - an Indigenous People in Sweden", Samediggi, accessed May 9 2017, http://www.samer.se/2137.

${ }^{4}$ Jorn Madslien, "Russia's Sàmi Fight for Their Lives", BBC.co.uk, December 21, 2006, http://news.bbc.co.uk/2/hi/business/6171701.stm.

${ }^{5}$ Kirsti Strom Bull, "Sàmi Reindeer Herders' Herding Rights in Norway from the Nineteenth Century to the Present Day", Indigenous Rights in Scandinavia: Autonomous Sàmi Law, ed. Christina Allard and Susann Funderud Skogvang, New York: Routledge, 2016, 84.

6 "The Sámi - an Indigenous People in Sweden", Samediggi, accessed May 9 2017, http://www.samer.se/2137.
} 
traditional livelihood of reindeer hunting; the impact of tourism, forestry, mining and hunting on Sámi homelands; the right to education and government services in Sámi languages; and autonomous or semi-autonomous government within the countries of Sápmi, which has achieved varying degrees of success. Questions of a unified, cohesive identity across four separate states are also paramount, especially with regards to the free movement of reindeer ${ }^{7}$.

The definition of being Sámi, for an individual person, can be complicated. Generally, the status is determined by two steps - self-identification, and speaking a Sámi language, or having a parent or grandparent who speaks a Sámi language ${ }^{8}$. However, some people of Sámi ancestry may not self-identify as Sámi, regardless of their language history, and are thus not counted. Conversely, those of Sámi ancestry from several generations back, or who have both Sámi and non-Sámi heritage, are ineligible to claim Sámi status and may not even be aware of their lineage, or may have lost access to their language in previous generations, thus making the definition highly problematic. This can also complicate the definition of a Sámi film, as the identity of the filmmakers or producers may be in question.

\footnotetext{
${ }^{7}$ Christina Allard and Susann Funderud Skogvang, "Introduction" , Indigenous Rights in Scandinavia: Autonomous Sàmi Law, ed. Christina Allard and Susann Funderud Skogvang, New York: Routledge, 2016, 4.

${ }^{8}$ Tanja Joona, "The Definition of a Sàmi Person in Finland and its Application", Indigenous Rights in Scandinavia: Autonomous Sàmi Law, ed. Christina Allard and Susann Funderud Skogvang, New York: Routledge, 2016, 156.
} 


\section{Chapter 2: Literature Review}

The field of Indigenous film study (as opposed to study of the portrayals of Indigenous people on film) is predominantly composed of recent writing; while some academic books and articles on the subject were published as early as the 1970s, especially in Australia ${ }^{9}$, the majority of works appear to have been published from the mid-2000s ${ }^{10}$. Writing on Sámi film, academic and otherwise, has been minimal, particularly in English; the examples in this review are mostly limited to textual readings of specific films, usually Nils Gaup's The Kautokeino Rebellion (2008) and especially Pathfinder (1987), due to its status as the first Norwegian film to be distributed in the United States and its Academy Award nomination ${ }^{11}$. Where political and economic status is concerned, as well as demographic information, I have used resources from the national governments of Sápmi's countries, and Sámi organizations within these countries. Websites of non- and intergovernmental organizations, particularly those which handle Indigenous and human rights issues, also provided an overview of the Sámi community within the four countries and in Sápmi as a whole.

In finding films, the Sámi Film Database provided the largest number by far, though it lacks entries from the more recent years and for certain films that had not come to the attention of the Skábmagovat Festival (which is explained in more detail later in this thesis). Other sources for films were programs from the Dellie Maa Festival and later years of Skábmagovat (which are

\footnotetext{
9 "Research - Journal Articles", Monash University, accessed August 42017, http://artsonline.monash.edu.au/indigenous-film-bibliography/research-journal-articles/.

10 "Results for 'indigenous film'", Ryerson University Library, accessed August 42017, https://ryerson.summon.serialssolutions.com/search?s.q=indigenous+film\&spellcheck=true\#!/search?ho=t\&l=en\&q =indigenous $\% 20$ film.

11 "Academy Awards Database", Academy of Motion Picture Arts and Sciences, accessed May 92017, http://awardsdatabase.oscars.org/search/results .
} 
not yet listed on the festival's website), the personal webpages of filmmakers, IMDB, the International Sámi Film Institute, and the Swedish Film Institute Database.

\section{1: Films and Their Content}

Indigenous empowerment is a central theme of the film analyses, focusing on how stories are told from within Sámi communities, rather than commentary on Sámi people from outsiders. In practice, this is mostly explored in Nils Gaup films, which have achieved great commercial success. In "Reclaiming the Past: On the History-Making Significance of the Sámi Film The Kautokeino Rebellion", Cato Christensen discusses how Gaup's 2008 film transforms the discussion surrounding a significant event in Sámi history, portraying the rebellion from a Sámi perspective rather than the mainstream Norwegian discourse. It addresses previous portrayals of Sámi characters in Nordic film, generally inaccurate, and the current efforts to reclaim Indigenous history and folklore, shifting "cultural memory" in the mainstream perception. Gunnar Iversen ("Learning From Genre: Genre Cycles in Modern Norwegian Cinema") describes the "indigenous [native to Norway, not Indigenous people] genre film", which he states came to prominence in the 1980s, focusing on Nils Gaup's films, particularly Pathfinder (Ofelas in Sámi), perhaps the most famous Sámi film. The article includes a discussion of funding in the Norwegian state, a combination of private and national support, but in the context of genre film, not specifically Indigenous or Sámi work. Iversen categorizes the film as similar to an American Western, and writes of the film's reception, box office, and Gaup's subsequent career. Wendy Gay Pearson ("Once Upon a Time in a Land Far, Far Away") analyzes Ofelas, in the context of a "decolonialized" form of storytelling, as the film is made by a Sámi filmmaker and depicts the time before Scandinavian colonization. The film is compared to Ten Canoes (Australia) and Atanarjuat: The Fast Runner (Canada) as further examples of decolonialized film, telling a story 
from an Indigenous perspective. Tina K. Ramnarine, in "Sonic Images of the Sacred in Sámi Cinema" explores the portrayal of Sámi traditional religion (pre-Christian) in the film Ofelas through the film's soundtrack, most notably in the use of drumming and joik (Sámi singing style). The article includes a history of joik, emphasizing its diverse use in the modern era. Ramnarine juxtaposes the use of these symbols as tourist items in real life alongside their portrayal as sacred in the film. The article also addresses the modern Sámi view of a pan-Sámi identity, transcending national borders, and the ways that the Sámi people fit, and do not fit, into this concept. Anne Yr Skardhamar traces the portrayal of Sámi people and culture in Nordic films, from 1929 to 2007, in "Changes in Film Representations of Sámi Culture and Identity." She discusses early stereotypes of Sámi people, including their portrayal as "childlike", as well as the later romanticization of Sámi culture and concern for its disappearance, both from mainstream Norwegian culture. She then contrasts these portrayals with two Sámi films, Ofelas and The Kautokeino Rebellion (1987 and 2008 respectively, both Nils Gaup), stating that they have left colonial discourse behind. The films no longer romanticize, or denigrate, the Sámi people, viewing historical events and folklore from a Sámi perspective and raising questions of Sámi rights in the past and today.

The academic work available on the subject of the Sámi film industry, or media in general, is also fairly sparse. In Representing 'Race': Racisms, Ethnicities and Media (London: Sage Publications Ltd., 2005), John Downing and Charles Husband discuss different aspects of Indigenous media, including political concerns, the problem with "good" versus "bad" representation, and infrastructure; Sámi media is discussed in a chapter on Indigenous empowerment through media portrayal, alongside the media of the Aboriginal people of Australia, and the Inuit of Nunavut. The authors discuss the development of the Sámi image in 
media, and how well-defined political autonomy tends to allow for greater freedom of Indigenous media. This work mostly refers to broadcast media, and is more concerned with the situation in Australia, but it is a helpful resource in finding works more deeply focused on Sámi media specifically. Sari Pietikainen's "To Breathe Two Airs: Empowering Indigenous Sámi Media" discusses the transnational nature of the Sámi media, and provides some historical and current political context for its existence, including some of the difficulties of broadcasting over a small population and wide geographic area. She also mentions the need for representation of Sámi issues from a Sámi perspective, free of stereotypes and in Sámi languages where possible. She writes about a drive for "Sáminess" across the four countries, treating the Sámi as one people regardless of borders. Her work covers Finland in-depth, briefly touching on Norway, and does not mention media in Sweden. It also questions which direction Sámi media will take in the future, as technology changes. "Contemporary Dynamics of Sámi Media in the Nordic States" (Lia Markelin and Charles Husband) addresses similar issues, as it draws a parallel between the separate developments of Sámi political autonomy, and the growth of Sámi media industries, in each Nordic country. It is primarily concerned with broadcast media, and does not explore film to a significant degree. This work is continued in Markelin and Husband's "The Sámi Media, State Broadcasting and TransnationalIndigeneity," which tracks the development of Sámi broadcast media alongside growing Sámi political rights in each Nordic country. Valerie Alia's The New Media Nation: Indigenous Peoples and Global Communication suggests that Sámi media is forced to be transnational due to varying levels of support from national governments, pointing specifically to Sweden's comparative lack of assistance. She explores the shared Sámi identity, which transcends Finnish, Norwegian, Russian or Swedish identity. Her work is also about broadcast media, primarily radio. Alia includes in-depth analysis of the Sámi history in 
Russia, in the context of other Russian indigenous populations, including a brief history of their media (which is a difficult topic to research). Veli-Pekka Lehtola's book The Sámi People:

Traditions in Transition looks at all aspects of Sámi life in the modern age, and briefly mentions film in the section on arts and media. Lehtola mostly speaks of Nils Gaup's career, including Beaivvas Theatre in Kautokeino, and Pathfinder (The Kautokeino Rebellion was not yet in production, but was mentioned as being in development). The book includes a brief summary of the works of Paul-Anders Simma, and a short paragraph mentioning the existence of the Skábmagovat Film Festival.

\section{2: Sámi History and Political Status}

Indigenous Rights in Scandinavia: Autonomous Sámi Law (ed. Christina Allard and Susann Funderud Skogvang) consists of an extraordinarily detailed and up-to-date view of Sámi law within the Nordic countries, as well as a comparison to Indigenous autonomy in other countries (such as Canada and New Zealand). Though it does not discuss media in particular, it does provide an excellent overview of the current legal and political situation, as well as ongoing debates about Sámi identity, international cooperation between Sámi groups in the Nordic countries, and land rights.

I also examined James Anaya's report, in his capacity as United Nations Special Rapporteur on the Rights on Indigenous Peoples, in 2011 on the subject of "The Situation of the Sámi People in the Sápmi Region of Norway, Sweden and Finland", as well as the 2016 report written by Victoria Tauli-Corpuz on the same topic when she served as Special Rapporteur. Overall, Anaya approves of the support given to the Sámi by Norway, Sweden and Finland. He advocates for stronger cross-border initiatives, including the adoption of a Sámi Nordic 
Convention. Anaya also expresses concern for land rights, linguistic survival, and continued education of tradition and culture for Sámi youth. Throughout the piece, he emphasizes the need for cohesion between the Sámi people, and the governments of the Nordic Sámi states, on existing and developing policies. Five years later, Tauli-Corpuz reports on issues of mining, identity, cross-border initiatives, and the development of the Nordic Saami Convention. While she acknowledges the developments in legislation and implementation of Sámi rights, she also feels that many of these initiatives could be improved, and makes recommendations for each country in several different fields. Prior to Tauli-Corpuz's visit, India Reed-Bowers prepared a report from the Swedish Sámi parliament, examining the same issues from a Sámi perspective. She includes a list of recommendations for land rights, recognition, and reconciliation, including a proposal for a Truth Commission in the style of other countries with Indigenous populations. Also writing for the United Nations, Eva Josefson gives a description of how the Sámi parliament functions in each Nordic country, the parliament's application in Sámi advocacy, and its cooperation with national government. It clearly outlines the difference in each Nordic country, emphasizes partnership with Russia, and defines the areas deemed lacking in autonomy for Sámi people in each country and across Sápmi as a whole. Timo Koivurova provides commentary on preliminary efforts towards a Nordic Sámi Convention, an initiative intended to provide a consistent legal framework across the Nordic states, between the Sámi people and national governments. Koivurova addresses the notion that the Sámi people are indivisible across the four states (Russia is acknowledged if not included), and advocates for strong support of crossborder activity and unity, especially in regards to land rights and Sámi legal customs. Of particular note is Koivurova's concern for the protection of reindeer herding and fishing livelihoods in Sámi communities. Koivurova praises the joint effort between the Sámi 
parliaments and the three national governments, and declares the effort to be ambitious and precedent-setting, though he is concerned that it will not go ahead in its current form. (The convention was approved in December 2016, but has not been ratified by national governments.)

From the perspective of the Swedish government, a report submitted by the Swedish Equality Ombudsman details the issues facing Sámi people in Sweden, both in terms of legal discrimination, and discrimination outside of the law, such as in the workplace. The Ombudsman concludes that legislation and official practices could be more comprehensive, including the ratification of ILO 169, an international convention on Indigenous rights by the International Labour Organization; access to education in Sámi languages; the involvement of Sámi people in decisions affecting them. The report also states that an attitude of racism and prejudice still exists in some aspects of Swedish society, particularly health care and the workplace, to which they suggest "mutual knowledge development". The document includes historical background information to add context for the modern situation.

For coverage of the Sámi in Russia, Vadislava Vladimirova's "Contested Political Representation of the Sámi in the Kola Peninsula, Northwest Russia" provides an account of the changes in legislation and in practices towards the Sámi and other "small-numbered peoples of the North" in the Russian Federation since the country's 1991 establishment. Due to shifting laws and varied implementation, even those protections promised by the state or region are not necessarily applied in reality, leaving the Sámi people divided and without the needed support of local administrations. 


\section{Chapter 3: Sámi Film}

What qualifies as a Sámi film? According to the Sámi Film Database, managed by the Friends of Sámi Art in conjunction with the Skábmagovat Film Festival, a film is considered a Sámi production if the central creators (for example, the director, producer or screenwriter) of the film include Sámi people ${ }^{12}$. In an interview, Kaija Anttonen, a translator who has worked with the Skábmagovat festival and the Sámi Film Database, clarified that a Sámi film was defined by the creative influence on the film; a film with a Sámi producer (individual or company), director or screenwriter would qualify. A film starring a Sámi actor, or concerning Sámi issues, however, would not automatically be included ${ }^{13}$. It should be noted that films with non-Sámi filmmakers could be included, if they were made by a Sámi production company or institution.

The oldest film included in the Sámi film database is Poazzovazzi (Antero Ruuhonen, Finland, 1963). Prior to this, film productions appear to have been made about the Sámi community by external filmmakers, rather than originating from filmmakers within the community. From then on, production was sporadic throughout the rest of the twentieth century. A full three hundred of the three hundred and twenty-three films in the Sámi Film Database, and all but four of the films I found outside of the Database, were made subsequent to the year 2000. The growth of digital media and resulting ease and reduced cost of filmmaking, as well as the formation of institutions like the International Sámi Film Institute, are likely catalysts for this sudden increase.

\footnotetext{
12 "The Sàmi Film Database", Skábmagovat.fi, accessed 9 May 2017, http://Skábmagovat.fi/Skábmagovat_2014/?page_id=6220.

${ }^{13}$ Kaija Anttonen in Skype interview with the author, April 18, 2017.
} 
In addition to exhibition within Sápmi, Sámi film has played at film festivals around the world, including several Indigenous events, such as Toronto's ImagineNative. One film, Pathfinder (Nils Gaup, 1987), became Norway's Best Foreign Language Film submission to the Academy Awards, the only film in a Sámi language to have achieved this distinction; it was nominated in that category, but did not win. Sámi film is frequently exhibited online, both on Sámi-focused websites (notably Nuoraj.tv) and general video-sharing sites like YouTube.

Sweden has the second-largest population of Sámi people globally (around 20,000 people $)^{14}$ and the largest general population of the Nordic countries ${ }^{15}$, possesses a welldeveloped film industry with a long history, and has the largest output of films in Northern Europe $^{16}$. Why, then, does Sweden historically lag in both the total number of Sámi films produced, and in the number of institutions available to Sámi filmmakers?

The historical attitude towards the Sámi people, particularly with regards to land, is markedly different between the three Nordic countries. Where the recognition of Sámi autonomy and Indigenous status is strongest, as in Norway and Finland, there tend to be more institutions and services available to Sámi artists, and thus more opportunity to create films exists. Sweden, which lags in recognition of Sámi issues and provides far less of an institutional framework for potential filmmakers, not only produces fewer films from Sámi artists or communities, but has a far greater proportion of filmmakers who must finance their films independently or through private funds (while state or public resources finance many productions in Finland and Norway). Though the trend of the disparity in films appears to be evening out in recent years, especially

\footnotetext{
14 "The Sámi - an Indigenous People in Sweden", Samediggi, accessed May 9 2017, http://www.samer.se/2137.

15 "Country Comparison: Population", CIA World Factbook, last updated July 2016, https://www.cia.gov/library/publications/the-world-factbook/rankorder/2119rank.html.

${ }^{16}$ Movie Production Countries, The Numbers, accessed May 9 2017, http://www.thenumbers.com/movies/production-countries/.
} 
with the prospect of expansion for the International Sámi Film Institute and ratification of a panNordic Sámi convention, Sweden still maintains a lack of institutional support that affects the production of films in its Sámi communities, a problem tied up in the historical issues, and political status, of the Sámi people. 


\section{Chapter 4: Analysis of Sámi Films and Institutions}

Sámi film is a small enough corpus that it is possible to catalogue, if not every film ever made, then at least the vast majority of the films that have been produced. The bulk of the films I found were listed on the Sámi Film Database (SFD). However, the database is incomplete, and many Sámi films are missing from the website. This issue was most significant when looking for films from 2014-2017, as the site had not yet catalogued them. I discovered extra films by searching the databases of the Swedish and Norwegian Film Institutes, the personal websites of Sámi filmmakers (or those of their companies), and programs from Skábmagovat and Dellie Maa. According to Skábmagovat's Kaija Anttonen, the database mostly draws information from the festival's programs, though some external research is conducted, and filmmakers are also free to submit their own information. Though works by Sámi filmmakers are included, the database may also extend to non-Sámi filmmakers, if the films are made by Sámi production companies or institutions. Information is verified from databases like IMDB, from distributors, and from filmmakers or production teams. Some films may also be passed over because it is not known that the creators are Sámi, or because the film is simply assumed to be from the country of production with no further specification. Due to staffing issues, the database has also not been updated since 2014 or 2015, though some more recent films may be found in the Skábmagovat $\operatorname{programs}^{17}$.

Despite these challenges, broad patterns in Sámi filmmaking can be drawn from the database, and from these external sources. Length and genre of film are easily discerned -- 100 of the database's films were documentaries (predominantly short form), 194 were short films, and twelve were features, while seven were listed as children's films. Nine were listed as TV

\footnotetext{
${ }^{17}$ Kaija Anttonen in Skype interview with the author, April 18, 2017.
} 
productions, which are also sometimes exhibited at Sámi film festivals, including Skábmagovat, Riddu Riddu and Dellie Maa ${ }^{18}$. Outside of the database, there were fifty documentaries, eight features, seventy-one shorts, seven TV productions, one "remix," twenty music videos, and three films labelled as "experimental." Fifteen films did not have a specified genre, all of them outside the database. Genre was determined by the descriptions from the database, program, or filmmaker's description, even when the categorization appeared ambiguous.

It is also possible to discern differences in film production between the four countries of Sápmi. The Database contained 113 films from Norway, thirty-eight from Sweden, 144 from Finland and one from Russia. Films not listed in the Database included forty-nine from Norway, forty-one from Sweden, fifty-three from Finland and two from Russia. Thirteen films in the Database and two outside the Database were co-productions between countries within Sápmi, of which nine and one included Sweden, respectively; six in the Database were co-productions between Sápmi and non-Sápmi countries, all of them in Europe, of which three films included Sweden. Outside the database, there are two collaborations between Sápmi and non-Sápmi countries, which can be attributed to the director Nils Gaup, who has worked with studios in Hollywood and Europe (though usually in collaboration with at least one country in Sápmi). One film was described as from Sápmi without further specification. Several films were marked as exclusively from non-Sápmi countries (two in the Database and ten outside of the Database). All but two are the work of Elle-Maija Tailfeathers, a filmmaker of Blackfoot and Sámi descent who works in both Canada and Sápmi ${ }^{19}$. Any collaborations with Canadian companies and organizations also refers to Tailfeathers' work. The remaining films are from NATIVE Slam, a

\footnotetext{
${ }^{18}$ In Scandinavia, the gap between television and theatrical film is not as strongly defined as in North America many theatrical films, including Bergman's Scenes from a Marriage and the Swedish adaptation of Stieg Larsson's Millennium Trilogy, were initially aired on television before their theatrical run.

19 "Elle-Maije Tailfeathers", ellemaijetailfeathers.com, accessed May 9 2017, http://elle-maija-tailfeathers.com/
} 
project based in New Zealand (and credited to that country) involving collaborations between Indigenous filmmakers from around the world. In some cases, information on national origin was not available; this applied to six films in the Database and fifteen outside of the database. Clearly, Finland and Norway far outrank Sweden in raw number of films produced, though the slightly more even figures outside of the Database (remembering that many of the missing films from the Database are from recent years) suggest that the number of films in each country are coming closer together over time.

The number of films produced does not necessarily account for the size of a film industry. To obtain a clearer idea of Sámi filmmaking, it is necessary to take a look at the institutional infrastructures available within the four countries. First, I catalogued every organization or individual which was listed as funding a Sámi film, and counted the number of films attributed to each entity (including joint funding). I gathered information on each funding body, where available, from websites belonging to institutions or artists. I then sorted the organizations into rough categories of Private Company, State Broadcaster, Individual, Non-Profit, Music Label, Mixed (multiple sponsors), Educational, and Unknown, which included both cases where the film's funding was not specified, and funding that was named but had no information available. Thereafter, I compiled the number of films produced in each category, further sorted by country or by combination of countries. This information is available in Appendix D and Appendix E. It was my hope to determine the general pattern of film funding in the Sámi communities of each country.

The numbers in these categories proved very revealing. In the database, Finland's films were mostly from educational institutions (ninety-six films), private companies (fourteen films), and non-profits (thirteen films). Norway's were a mixture of private companies (twenty-seven 
films), state media (twenty-six films), films of mixed funding (seventeen films), and educational institutions (thirteen films). In Sweden, the largest category was private companies at fourteen films, followed by individuals at nine films, with state media and mixed funding tied at five each. (Many of these private companies were also small-scale, and driven by the filmmakers themselves.) Educational institutions produced only two films, while one film came from a nonprofit. This points to a stronger support for filmmaking infrastructure for the Sámi communities in Finland and Norway, both directly through the state media, and through support for film education and organizations, which suggests that Sámi filmmakers in Sweden are more likely to turn to private companies or personal resources.

This trend of weaker institutional support in Sweden appears to continue in films outside of the Database, though the numbers have less of a discrepancy. Out of 175 total films listed, there are forty-one films from Sweden, forty-nine from Norway, and fifty-three from Finland, as well as two from Russia, four joint productions (two between Sápmi countries, and two between Sápmi and non-Sápmi countries), and one labeled as "Sápmi" without specifying countries. There ten non-Sápmi films (eight from Blackfoot/Sámi filmmaker Elle-Maija Tailfeathers, and two from the New Zealand-based NATIVE Slam project). Fifteen have no country specified. One reason for this could be the lack of updates in the Database from 2014-2017, suggesting more films from Sweden in recent years, and indeed twenty-five of the forty-one Swedish films that I have found outside of the database are from 2013 or later. However, the data here is slightly faultier, often due to lack of documentation in the source websites and programs. Where the producer can be identified, the films still have the general pattern of funding that they have in the database -- Sweden is mostly from private companies (ten films), Norway from state media (thirteen films) and private companies (ten films), and Finland from private companies (seven 
films) and educational institutions (fifteen films). Though filmmakers in all three countries work with private companies, Norway and Finland both offer a greater number of films, and produce works in an institutional context that appears to be lacking in Sweden. However, the vast majority of films from all countries are of unknown funding, thus it cannot be said that the patterns take all films into account. (This information is summarized in Appendix E.)

Though a similar number of educational institutions producing Sámi film exist in Norway (three institutions, one specifically Sámi-focused, all located in Sápmi), Sweden (two institutions, neither specifically Sámi-focused, both located in Sápmi), and Finland (three institutions, one Sámi-focused, two in Sápmi), the Finnish and Norwegian institutions are generally older and produce a far greater number of films. The Sámi film festivals, including Riddu Riddu (Norway, est. $1991^{20}$ ), the Sámi Film Festival/Sámi Easter Festival (Norway, est. $1971^{21}$ ), Skábmagovat (Finland, est. 1999 22 ), and Dellie Maa (Sweden, est. $2013^{23}$ ), also point to a much more longstanding establishment in Norway and Finland. Overarching film institutions devoted to Sámi film, the International Sámi Film Institute and Skábma - the Indigenous People's Film Centre (a film resource centre operating within the Sámi Parliament of Finland, which helps locate funding, educate filmmakers, and provide distribution and screening opportunities) ${ }^{24}$, are located in Norway and Finland respectively. Aside from the number of films produced by each country, there is clearly a much stronger framework of filmmaking aimed at, or enabling, Sámi filmmakers to produce films.

\footnotetext{
20 "The History of Riddu Riddu", Riddu Riddu, accessed May 9 2017, http://riddu.no/en/about-riddu-riddufestivala/history-riddu-riddu.

21 "Doing it the Sàmi Way: Beassasmarkanat in Norway", The Nordic Page, last updated January 5, 2017, http://www.tnp.no/norway/exclusive/4466-doing-it-the-Sàmi-way-beassasmarkanat-in-norway.

22 "Skábmagovat Film Festival History", Skábmagovat.fi, accessed May 92017, http://www.Skábmagovat.fi/Skábma_vanhat/Skábma_2010/historia_en.htm .

23 Oskar Östergren in Skype discussion with the author, February 20, 2017.

24 "Skábma - the Indigenous Peoples' Film Centre", Samediggi, accessed May 92017, http://www.samediggi.fi/index.php?option=com_content\&task=blogcategory\&id=167\&Itemid=311\&lang=english.
} 
The International Sámi Film Institute was founded in 2007 to promote filmmaking in the Sámi languages. Though the Institute seeks to work across the countries of Sápmi, and serves professional filmmakers in all four countries, the funding is primarily Norwegian, including grants from the Norwegian Ministry of Culture and the Norwegian Sámi Parliament ${ }^{25}$. According to Oskar Östergren, founder of the Dellie Maa festival and board member at the ISFI, future initiatives include a more international distribution of funding between the countries of Sápmi ${ }^{26}$, supported by the organization's restructuring from the International Sámi Film Centre to the International Sámi Film Institute in $2014^{27}$-- which is compliant with their support from Norway while still allowing them to exist as a "national and international" institution ${ }^{28}$. Östergren suggested that non-Sámi funding bodies should include Sámi cultural perspectives in their decisions, as cultural context is often essential for understanding a potential film's content, and may contribute towards approval or denial of funding for a proposed project. ${ }^{29}$ Aside from the ISFI, there is no state-funded agency specifically aimed towards funding Sámi filmmakers' work, or promoting Sámi content in the Nordic countries.

\footnotetext{
25 "International Sámi Film Institute", International Sámi Film Institute, accessed May 9 2017, http://www.isfi.no/eng/about/isfi/.

${ }^{26}$ Oskar Östergren in Skype discussion with the author, February 20, 2017.

27 "International Sámi Film Institute", International Sámi Film Institute, accessed May 9 2017, http://www.isfi.no/eng/about/isfi/.

${ }^{28}$ Ibid.

${ }^{29}$ Oskar Östergren in Skype discussion with the author, February 20, 2017.
} 


\section{Chapter 5: Sámi Rights by Country}

The current coverage of Sámi rights varies in each country of Sápmi. Norway is only one of five countries, and the only one in Sápmi, to have ratified treaty ILO 169. Christina Allard (a professor at Luleå Technical University), in discussing Nordic law in general, suggests that this contributes to a greater drive for Norway to "come to terms with its colonial past," paving the way for legislation that permits the Sámi people "substantial influence in the drafting of legislation affecting them." Allard has praised the Finnmark Act of 2005, which enables some implementation of ILO 169 in the traditionally Sámi area of Finnmark in Northern Norway (as opposed to the entire country), ${ }^{30}$ by giving the rights to the land to the people of Finnmark. Finland also has taken steps towards a "Sámi homeland region...with cultural and linguistic autonomy," ${ }^{31}$ but has reached difficulty with defining Sámi identity.

Allard attributes Norway's progress to a tradition of more independent courts, as opposed to Sweden and Finland. ${ }^{32}$ The organization of Sweden during the seventeenth century, around a far more centralized property system ultimately based around a king ${ }^{33}$, was echoed by Finland as part of the Swedish Empire at the time. Norway, by contrast, was far more removed as a province of Denmark, and was thus left to determine many of its property laws alone, especially in remote areas, leading to a great deal of common land. ${ }^{34}$ Land rights, crucial to Sámi reindeerherding and fishing practices, are addressed to some extent in all three countries, but Norway alone is party to ILO 169, which asks signatory countries to interpret laws according to the

\footnotetext{
${ }^{30}$ Christina Allard, "Some Characteristic Features of Scandinavian Laws and their Influence on Sámi Matters," Indigenous Rights in Scandinavia: Autonomous Sàmi Law, ed. Christina Allard and Susann Funderud Skogvang, New York: Routledge, 2016, 51.

${ }^{31}$ Ibid.

${ }^{32}$ Allard, 54-55.

${ }^{33}$ Ibid, 56.

${ }^{34}$ Ibid, 56.
} 
"custom" of Indigenous peoples (though Finland had a draft proposal to ratify the convention as of 2015). ${ }^{35}$ These characteristics illustrate Norway's very different interpretation of Sámi law, both due to historical and to modern conventions, which ultimately allows greater freedom for Sámi people.

In terms of recognition as a distinct people, the Sámi are recognized as Indigenous by the Russian Constitution, though this has proved to have little practical effect. They have been recognized in Sweden as Indigenous since $1977^{36}$ and as "ethnic minorities" since $1999 .{ }^{37}$ It is also important to distinguish that the recognition as "Indigenous" is not in the Swedish Constitution, where they are simply defined as a "people", while Norway and Finland recognize them as Indigenous ${ }^{38}$. This is a problem for the Sámi people, as recognition as an "ethnic minority" or "people" does not necessarily obligate Sweden to follow treaties and agreements pertaining to the treatment of Indigenous peoples. Another issue in Sweden is that laws related to hunting and fishing tend to distinguish between Sámi people of traditional villages and those outside of the villages, leaving many Sámi with few or no hunting or fishing rights and disconnecting them from their traditional livelihood. ${ }^{39}$

In Finland, since its independence from the Russian Empire in 1917, the country has had the ability to develop its own policy on Sámi rights. Finland is currently in the process of

\footnotetext{
35 Ibid, 60.

36 "Discrimination of the Sámi - the Rights of the Sámi from a Discrimination Perspective", Ombudsmannen mot etnisk diskriminering [Ombudsman against ethnic discrimination], Stockholm: Lenanders Grafiska AB, $2008,5$.

${ }^{37}$ Ibid, 14.

${ }^{38}$ IndiaReed-Bowers, "Preparatory Report from the Sámi Parliament in Sweden/Sámediggi/Sámedigge/Saemiedigkie/Sametinget for the United Nations Special Rapporteur on the Rights of Indigenous Peoples, Ms. Victoria Tauli-Corpuz, prior to her 2015 August visit to Sápmi and Sweden", Samediggi, August 2015, https://www.sametinget.se/92639.

${ }^{39}$ Eivind Torp, "Sámi Hunting and Freshwater Fishing Rights in Swedish Law", Indigenous Rights in Scandinavia: Autonomous Sàmi Law, ed. Christina Allard and Susann Funderud Skogvang, New York: Routledge, $2016,111-112$.
} 
ratifying ILO $169 .^{40}$ In terms of land rights, though most of the Sámi homeland is officially owned by the Finnish state, and the government and Sámi officials are currently in negotiation for its common use. However, a recent Mining Act (2011) contained special considerations pertaining to the impact on the Sámi as a result of gold panning or mining, ${ }^{41}$ and is regarded as a positive step forward by UN Special Rapporteur for the Rights of Indigenous Peoples Victoria Tauli-Corpuz in her report of 2016. Sámi are recognized as Indigenous in Finland, ${ }^{42}$ but their determination of Sámi identity is more restrictive than Norway and Sweden, determined by ability to vote in the Sámi Parliament, which can lead to exclusion. ${ }^{43}$ Definitions of Sámi-ness by language can also be problematic, as many people deliberately suppressed or were forced to suppress their Sámi language in previous generations, leaving a disconnect. ${ }^{44}$

Language rights themselves vary across Sápmi - Russia has no provisions for Sámi languages. The "ethnic minority" distinction in Sweden gives Sámi people the right to education, eldercare, and contact with authority in Sámi, though education is limited by location (within traditionally Sámi areas only) and availability of teachers, and contact with authority is only available in Sámi in certain municipalities. ${ }^{45}$ Norway has adopted an Action Plan for Sámi Languages, which was praised by Corli-Taupuz in the 2016 report, ${ }^{46}$ but has faced difficulty in implementing the tenets of the plan. In Finland, Sámi-language education is guaranteed in the

\footnotetext{
${ }^{40}$ Tanja Joona, "The Definition of a Sàmi Person in Finland and its Application", Indigenous Rights in Scandinavia: Autonomous Sàmi Law, ed. Christina Allard and Susann Funderud Skogvang, New York: Routledge, 2016, 155.

${ }^{41}$ Victoria Tauli-Corpuz, "Report on the Human Rights Situation of the Sámi People in the Sápmi Region of Norway, Sweden and Finland", United Nations General Assembly Human Rights Council Thirty-Third Session, Agenda Item 3, 9 August 2016, http://unsr.vtaulicorpuz.org/site/index.php/documents/country-reports/155-reportSápmi-2016.

${ }^{42}$ Ibid.

${ }^{43}$ Joona, 156.

${ }^{44}$ Ibid, 170

${ }^{45}$ Victoria Tauli-Corpuz, "Report on the Human Rights Situation of the Sámi People in the Sápmi Region of Norway, Sweden and Finland", United Nations General Assembly Human Rights Council Thirty-Third Session, Agenda Item 3, 9 August 2016, http://unsr.vtaulicorpuz.org/site/index.php/documents/country-reports/155-reportSápmi-2016.

${ }^{46}$ Ibid.
} 
"Sámi homeland", but not outside of it, and faces the same logistical problems as in Sweden, though some distance learning is available to ameliorate the situation. ${ }^{47}$ However, a Sámi language revitalization programme has been approved by the Finnish government in 2014, with the hope of increasing the number of Sámi speakers by $2025 .^{48}$

The Nordic Sámi Convention, drafted by an "Expert Group"49 composed of representatives from the governments, and the Sámi communities, of each Nordic country, seeks a shared approach to Sámi rights and infrastructure between the three states. Most prominently, it explicitly names the Sámi as a distinct Indigenous people with a right of self-determination. ${ }^{50}$ The 2005 draft of the convention includes consideration for Sámi legal customs, the existence and powers of Sámi parliaments, the right to a Sámi region (with "traditional use of land and water", as well as natural resources), and provisions for language, culture, goverment services, education and media in a Sámi context. ${ }^{51}$ The Russian Federation, it is hoped, would be added to negotiations following the adoption of the Convention in the Nordic countries. ${ }^{52}$ Though the draft was initially presented in $2005,{ }^{53}$ and was completed in its final form in December $2016,{ }^{54}$ it has yet to be ratified by Nordic national governments.

\footnotetext{
${ }^{47}$ Ibid.

${ }^{48}$ Ibid.

${ }^{49}$ Leena Heinämäki, "The Rapidly Evolving International Status of Indigenous Peoples: The Example of the Sámi People in Finland", Indigenous Rights in Scandinavia: Autonomous Sàmi Law, ed. Christina Allard and Susann Funderud Skogvang, New York: Routledge, 2016, 198.

${ }^{50}$ Ibid.

51 "Nordic Saami Convention", Regjeringen.no [Norwegian government website], accessed 9 May 2017, https://www.regjeringen.no/globalassets/upload/aid/temadokumenter/Sámi/Sámi_samekonv_engelsk.pdf .

52 Timo Koivurova, "The Draft for a Nordic Saami Convention", European Yearbook of Minority Issues 6 (2008): 103, http://www.arcticcentre.org/loader.aspx?id=04e81223028e-4315 a669-cb8830b22d6b.

${ }^{53}$ Heinämäki, 198.

54 "Agreement on the Sámi Draft Convention Reached", Ministry of Justice of Finland, December 21, 2016, http://oikeusministerio.fi/en/index/currentissues/tiedotteet/2016/12/pohjoismaisestasaamelaissopimuksestaneuvottel utuloshallitusvieratifioitavaksi.html.
} 
Outside of the Nordic countries, the situation in Russia gives few rights for Sámi people in practice, though the laws do grant some framework for self-autonomy. The Constitution offers protection for the "obshchiny [communities] of small-numbered peoples of the far North, Siberia and the Far East of the Russian Federation." ${ }^{55}$ The Sámi people are one of many Indigenous groups in Russia, and are small in number, a significant contrast to the situation in the Nordic countries, where the Sámi are one of few indigenous or official minority groups, and form larger communities. Article 18 of the Russian Constitution, concerning the protection of Indigenous rights, was abolished in 2004, but promised that "the members of obshchiny of small-numbered peoples enjoy privileges, established by the federal legislation and the legislation of the subjects of the Russian Federation in order to protect their aboriginal environment, to preserve and develop their traditional ways of life and economic activities." ${ }^{56}$ Article 69 of the Russian Constitution states that "the Russian Federation shall guarantee the rights of the indigenous small peoples according to the universally recognized principles and norms of international law and international treaties and agreements of the Russian Federation," ${ }^{57}$ though at this time, Russia has not yet ratified ILO $169,{ }^{58}$ and specific legislation regarding land protection and language rights does not exist.

Russia's unstable legal environment and complex bureaucracy undermine the implementation of such laws. On Guarantees of the Rights of the Indigenous Small-numbered Peoples of the Russian Federation (ratified 1999), contains Article 13, which allows for support of living areas and traditional livelihood through establishing quotas of Indigenous

\footnotetext{
${ }^{55}$ Vladislava Vladimirova, "Contested Political Representation of the Sámi in the Kola Peninsula, Northwest Russia", Re: mindings. Co-Constituting Indigenous/Academic/Artistic Knowledges, ed. Johan Gärdebro, May-Britt Öhman, Hiroshi Maruyama, Stockholm: Vulkan, 2014, 218.

56 Ibid.

57 "Chapter 3. The Federal Structure", The Russian Constitution, accessed May 92017, http://www.constitution.ru/en/10003000-04.htm.

${ }^{58}$ Vladimirova, 219.
} 
representatives in regional governments (this was later abolished in 2004). Instead, article 7 , paragraph 6 (pg 220) gives these powers back to municipal administrations -- the theory is that regional self-governance will provide equality to all, including Indigenous peoples. Per Vladimirova, the laws "bear the character of recommendations" and put the management of Indigenous organizations and economic practices into the hands of regional and municipal administrations, forcing Indigenous groups to comply with local administrators. ${ }^{59}$ Foreign (often Fennoscandic) NGOs have also tried to help in Kola Peninsula, achieving some political improvements (such as establishing commitees), but creating little economic improvement. In 2010, the Kola Sámi Parliament was created to advocate for self-governance and political rights, but according to Vladimirova, it suffers from limited participation. She characterizes this as "vernacular politics," more akin to a social network than a legal system. ${ }^{60}$

In this disparity, it is also possible to trace the divergence of respective film industries across Sápmi. Generally, where Sámi autonomy and language rights are stronger, there is a greater framework for funding and creating Sámi film, such as film education, exhibition opportunities and production companies. Norway seems to have progressed the furthest in recognition of Sámi rights, especially with the ratification of ILO 169. Sweden, meanwhile, seems to lag behind Norway, with limited and disputed legal recognition of the Sámi. Finland, somewhere in the middle, bears some of the problems that Sweden has, but is in the process of implementing solutions to deal with many of them. The size of the film industry in each country corresponds to the relative freedom the Sámi have -- Norway and Finland both maintain Sámi institutions and infrastructure towards filmmaking that Sweden lacks, and each produces a far larger number of films. Furthermore, more of these films come from larger institutions such as

\footnotetext{
${ }^{59}$ Vladimirova, 220.

${ }^{60}$ Ibid.
} 
postsecondary educational organizations, state media, and non-profits, while Swedish films tend to be more inclined towards private funding (from companies or from individuals), which suggests greater support overall for Sámi filmmaking in Norway and Finland. In Russia, which offers limited rights and recognition to the Sámi people, and even less implementation of said rights, there is almost no Sámi film to speak of.

Film production is a complex endeavour, requiring logistic and financial cooperation from a variety of parties. It is only when these factors come together that a film can be produced. While it is inaccurate to say that filmmaking is directly influenced by the legal and cultural standing of Sámi people within the Nordic countries, it is possible to make a case that increased recognition, legal and economic rights, and support for autonomy can lead to an environment in which a filmmaking industry, and the accompanying infrastructure, may flourish, and that the situation of filmmaking in each country parallels the general status of the Sámi people. With the creation of the International Sámi Film Institute, along with its intentions to even out funding across the Nordic countries, together with the prospect of ratification of the Nordic Sámi Convention, it is hopeful that more Sámi films will be produced, and that prospective filmmakers in Sápmi will have access to a stronger film industry, at a more comparable level in all three countries. 


\section{Appendix A: Sámi History and Facts}

\section{Population Statistics}

\begin{tabular}{|l|l|}
\hline Country & Population $^{61}$ \\
\hline Norway & 40,000 \\
\hline Sweden & 20,000 \\
\hline Finland & 8,000 \\
\hline Russia & 2,000 \\
\hline Other & \\
& 30,000 in North America ${ }^{63}$; European and \\
\hline Total & other statistics unknown \\
\hline
\end{tabular}

Sámi Language by Speaker

\begin{tabular}{|l|l|l|l|}
\hline Language $^{64}$ & Number of Speakers & Countries spoken in & Language Status $^{65}$ \\
\hline Akkala Sámi & 0 & Russian Federation & 9 (dormant) \\
\hline Inari Sámi & 300 & Finland & 2 (provincial) \\
\hline Kildin Sámi & 350 & Russian Federation & $8 \mathrm{~b}$ (nearly extinct) \\
\hline Lule Sámi & 2000 & Norway, Sweden & 2 (provincial) \\
\hline
\end{tabular}

\footnotetext{
61 "The Sámi - an Indigenous People in Sweden", Samediggi, accessed May 9 2017, http://www.samer.se/2137.

${ }^{62}$ Primarily USA, Canada, Ukraine, other former Soviet states; includes people of Sámi descent who are not necessarily Sàmi by official definition.

63 "About Báiki", Báiki: The International Sámi Journal, accessed May 9 2017, http://www.baiki.org/content/about.htm .

${ }^{64}$ Considerable debate has been undertaken as to how many Sámi languages exist, as opposed to dialects; I have chosen to follow the ISO classification, as listed on ethnologue.com.

${ }^{65}$ Taken from the EGIDS scale, a tool for measuring language endagerment; for further information, see https://www.ethnologue.com/enterprise-faq/what-egids-how-it-used.
} 


\begin{tabular}{|l|l|l|l|}
\hline North Sámi & 25,700 & Norway & 2 (provincial) \\
\hline Pite Sámi & 20 & Sweden & $8 \mathrm{~b}$ (nearly extinct) \\
\hline Skolt Sámi & 300 & Finland & 7 (shifting) \\
\hline South Sámi & 600 & Sweden & 2 (provincial) \\
\hline Ter Sámi & 350 & Russian Federation & $8 \mathrm{~b}$ (nearly extinct) \\
\hline Ume Sámi & 20 & Sweden & $8 \mathrm{~b}$ (nearly extinct) \\
\hline
\end{tabular}

Major Sámi Centres and Locations

\begin{tabular}{|l|l|l|}
\hline Place & Population & Country \\
\hline Inari & $6806^{66}$ & Finland \\
\hline Utsjoki & 1239 & Finland \\
\hline Jokkmokk & $5105^{67}$ & Sweden \\
\hline Kautokeino & $1386^{68}$ & Norway \\
\hline Rovaniemi & 62246 & Finland \\
\hline Lovozero & $2871^{69}$ & Russia \\
\hline
\end{tabular}

\footnotetext{
66"Municipality-Based Statistics Unit", Statistics Finland, accessed May 9 2017, http://www.stat.fi/tup/rajapintapalvelut/kuntapohjaiset_tilastointialueet_en.html.

67 "Population in the Country, Counties and Municipalities on 31/12/2016 and Population Change in 2016", Statistics Sweden, accessed May 9 2017, http://www.scb.se/en/finding-statistics/statistics-by-subjectarea/population/population-composition/population-statistics/pong/tables-and-graphs/yearly-statistics-municipalities-counties-and-the-whole-country/population-in-the-country-counties-and-municipalities-andpopulation-change/.

68 "Populaton and Area by Municipality", Statistics Norway, accessed May 9 2017, http://www.ssb.no/303784/population-and-area-by-municipality-sy-57.

${ }^{69}$ ЧИСЛЕННОСТЬ НАСЕЛЕНИЯ РОССИИ, ФЕДЕРАЛЬНЫХ ОКРУГОВ, СУБЪЕКТОВ РОССИЙСКОЙ ФЕДЕРАЦИИ, РАЙОНОВ, ГОРОДСКИХ НАСЕЛЕННЫХ ПУНКТОВ, СЕЛЬСКИХ НАСЕЛЕННЫХ ПУНКТОВ - РАЙОННЫХ ЦЕНТРОВ И СЕЛЬСКИХ НАСЕЛЕННЫХ ПУНКТОВ С НАСЕЛЕНИЕМ 3

ТЫСЯЧИ ЧЕЛОВЕК И БОЛЕЕ [Population of Russia, federal districts of the Russian Federation subjects, districts, urban localities, rural localities - administrative centers, and rural localities with population of 3000 people and more - 2010 Russian census], Всероссиская перепись населения [All-Russia Population Census] accessed May 9 2017, http://www.gks.ru/free_doc/new_site/perepis2010/croc/Documents/Vol1/pub-01-05.pdf.
} 


\begin{tabular}{|l|l|l|}
\hline Troms $\varnothing$ & 32774 & Norway \\
\hline Kiruna & 23167 & Sweden \\
\hline Karasjok & 1858 & Norway \\
\hline Hattfjelldal & 594 & Norway \\
\hline Arjeplog & 2876 & Sweden \\
\hline Tana & 668 & Norway \\
\hline Tysfjord & 1187 & Norway \\
\hline Arvidsjaur & 6442 & Sweden \\
\hline Nesseby & 919 & Norway \\
\hline Snåsa & 652 & Norway \\
\hline Neiden & 250 & Norway/Finland \\
\hline Östersund & 61745 & Sweden \\
\hline Lakselv & 2258 & Norway \\
\hline Alta & 14272 & Norway \\
\hline Kirkenes & 3498 & Norway \\
\hline Gällivare & 17956 & Sweden \\
\hline Enontekiö & 1874 & Finland \\
\hline Kåfjord & 299 & Norway \\
\hline Umeå & 231 & Norway \\
\hline Luleå & & \\
\hline Royrvik & & \\
\hline
\end{tabular}




\section{Appendix B: Sámi Film History, Exhibition and Institutions}

\section{Timeline of Sámi Film History}

- 1963: First Sámi film, according to the Sámi Film Database. Any films prior to this date appear to be filmed by external filmmakers about Sámi communities, rather than coming from within the communities. ${ }^{7071}$

- 1971: First Sámi Easter Festival. ${ }^{72}$

- 1982: First Sámi feature film, Skierri - Land of the Dwarf Birches. ${ }^{73}$

- 1987: Release of Ofelas, which would be nominated for an Academy Award for Best Foreign Language Film, ${ }^{74}$

- 1991: First Riddu Riddu festival. ${ }^{75}$

- 1999: First Skábmagovat, an Indigenous film festival hosted annually in Inari, Finland. ${ }^{76}$

- 2000: 300/323 films on the Sámi Film Database appear after this date.

- 2007: Establishment of International Sámi Film Centre in Kautokeino, NO. ${ }^{77}$

- 2013: Establishment of Dellie Maa, first Sámi film festival in Sweden. ${ }^{78}$

\footnotetext{
70"The Sàmi Film Database", Skábmagovat.fi, accessed May 9 2017, http://www.Skábmagovat.fi/Sàmifilm/movies/.

${ }^{71}$ Kaija Anttonen in Skype interview with the author, April 18, 2017.

72 "Doing it the Sàmi Way: Beassasmarkanat in Norway", The Nordic Page, last updated January 5, 2017, http://www.tnp.no/norway/exclusive/4466-doing-it-the-Sàmi-way-beassasmarkanat-in-norway.

73 "The Sàmi Film Database", Skábmagovat.fi, accessed May 9 2017, http://www.Skábmagovat.fi/Sàmifilm/movies/.

74 Academy Awards Database", Academy of Motion Picture Arts and Sciences, accesser May 92017, http://awardsdatabase.oscars.org/search/results.

75 "The History of Riddu Riddu", Riddu Riddu, accessed May 9 2017, http://riddu.no/en/about-riddu-riddufestivala/history-riddu-riddu.

76 "Skábmagovat Film Festival History", Skábmagovat.fi, accessed May 92017, http://www.Skábmagovat.fi/Skábma_vanhat/Skábma_2010/historia_en.htm.

77 "International Sámi Film Institute", International Sámi Film Institute, accessed May 9 2017, http://www. isfi.no/eng/about/isfi/.

${ }^{78}$ Oskar Östergren in Skype discussion with the author, February 20, 2017.
} 
Sámi Film Education

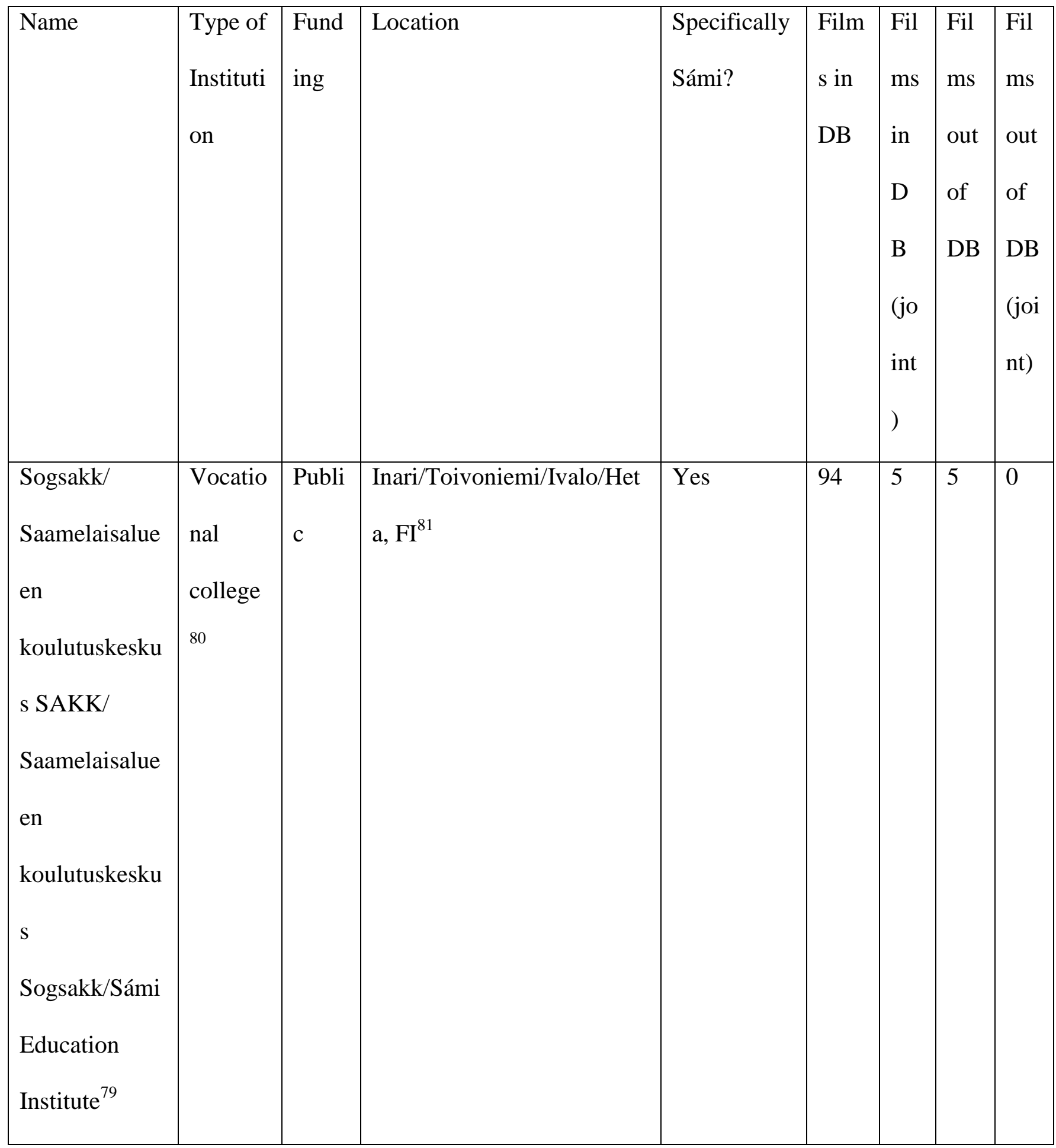

79 "The Sámi Education Institute", Saamelaisaluen koulutuskeskus, accessed May 9 2017, http://www.sogsakk.fi/index.php?lang=en.

${ }^{80}$ Ibid. 


\begin{tabular}{|c|c|c|c|c|c|c|}
\hline $\begin{array}{l}\text { Nordland } \\
\text { College of Art } \\
\text { and } \\
\text { Film/NKFS }{ }^{82}\end{array}$ & $\begin{array}{l}\text { Art and } \\
\text { media } \\
\text { college } \\
83\end{array}$ & & Kabelvåg, $\mathrm{NO}^{84}$ & No & 8 & 5 \\
\hline $\begin{array}{l}\text { Sámi } \\
\text { University } \\
\text { College }{ }^{85} / \text { Sám } \\
\text { i } \\
\text { allaskuvla/Sá } \\
\text { mi University } \\
\text { of Applied } \\
\text { Sciences }{ }^{86} / T h \\
\text { e Nordic Sámi } \\
\text { Institute }^{87}\end{array}$ & $\begin{array}{l}\text { Vocatio } \\
\text { nal } \\
\text { college }\end{array}$ & $\begin{array}{l}\text { Publi } \\
\text { c }\end{array}$ & Kautokeino, NO & Yes & 5 & 2 \\
\hline Academy of & Art and & Unkn & Banská Bystrica, SK ${ }^{88}$ & No & 1 & ( \\
\hline
\end{tabular}

\footnotetext{
81 Ibid.

82 "Welcome to Nordland kust og film- fagskole", Nordland Fylkeskommune, accessed May 92017, http://www.kunstfilm.no/english/.

${ }^{83}$ Ibid.

${ }^{84}$ Ibid.

85 "Sámi University College", UArctic, accessed May 9 2017, http://www.uarctic.org/memberprofiles/norway/8710/Sámi-university-college.

86" "Sámi University of Applied Sciences", Sámi Allaskuvla, accessed May 9 2017, http://samas.no/en.

87 "Research", Sámi Allaskuvla, accessed May 9 2017, http://samas.no/en/node/156.

88 "Institute of Documentary Film", Institute of Documentary Film, accessed May 92017, https://dokweb.net/database/organizations/about/3211aabf-8d4a-40bb-a1a1-82dd43babada/academy-of-arts-banskabystrica.
} 


\begin{tabular}{|c|c|c|c|c|c|c|c|c|}
\hline Arts & $\begin{array}{l}\text { media } \\
\text { college }\end{array}$ & own & & & & & & \\
\hline $\begin{array}{l}\text { Finnmark } \\
\text { University } \\
\text { College/Unive } \\
\text { rsity of } \\
\text { Troms } \emptyset^{89} / \mathrm{Hog} \\
\text { skolen i } \\
\text { Finnmark }\end{array}$ & $\begin{array}{l}\text { Univers } \\
\text { ity }\end{array}$ & $\begin{array}{l}\text { Publi } \\
\text { c }\end{array}$ & $\begin{array}{l}\text { Tromsø/Alta/Hammerfest/ } \\
\text { Narvik/Kirkenes/Harstad, } \\
\mathrm{NO}^{90}\end{array}$ & No & 2 & 1 & 2 & 0 \\
\hline $\begin{array}{l}\text { Helsinki } \\
\text { College } \\
\text { Stadia/Helsink } \\
\text { i Polytechnic } \\
\text { Stadia/Helsink } \\
\text { i Metropolia } \\
\text { University of } \\
\text { Applied } \\
\text { Sciences }\end{array}$ & $\begin{array}{l}\text { Polytec } \\
\text { hnic }\end{array}$ & & Helsinki, FI & No & 0 & 0 & 0 & 1 \\
\hline $\begin{array}{l}\text { Kalix } \\
\text { Folkhögskola }\end{array}$ & $\begin{array}{l}\text { Adult } \\
\text { Educati }\end{array}$ & $\begin{array}{l}\text { Publi } \\
\text { c }\end{array}$ & Kalix, SE $^{92}$ & No & 1 & 0 & 0 & 0 \\
\hline
\end{tabular}

89 "UiT2020: Towards a New Strategic Plan for UiT", University of Troms $\phi$, last updated January 29, 2015, https://en.uit.no/om/art?p_document_id=356184\&dim=179033.

90 "UiT Studiesteder", University of Troms $\phi$, accessed May 9 2017, https://en.uit.no/studiesteder.

91 "EVTEK and Helsinki Higher Vocational School Stadia in Finland Merge", The Connection, last updated January 27 2011, https://theconnection.ece.org/NewsItem/86.

92"Om Kalix" [about Kalix], Kalix Folkhögskola, accessed May 9 2017, http://www.kalix.fhsk.se/om-kalix/. 


\begin{tabular}{|c|c|c|c|c|c|c|c|c|}
\hline & on & & & & & & & \\
\hline $\begin{array}{l}\text { Tampereen } \\
\text { ammattikorke } \\
\text { akoulu TAMK }\end{array}$ & $\begin{array}{l}\text { Polytec } \\
\text { hnic }\end{array}$ & $\begin{array}{l}\text { Publi } \\
\text { c }\end{array}$ & Tampere, $\mathrm{FI}^{93}$ & No & 3 & 0 & 0 & 0 \\
\hline $\begin{array}{l}\text { Luleå } \\
\text { University of } \\
\text { Technology }\end{array}$ & $\begin{array}{l}\text { Univers } \\
\text { ity }\end{array}$ & $\begin{array}{l}\text { Publi } \\
\text { c }\end{array}$ & Luleå, SE ${ }^{94}$ & No & 1 & 0 & 0 & 0 \\
\hline
\end{tabular}

Sámi Film Festivals and Events ${ }^{95}$

\begin{tabular}{|l|l|l|l|}
\hline Name & Time, Frequency & Established & Location \\
\hline Dellie Maa & Autumn, annual & $2013^{96}$ & $\begin{array}{l}\text { Tärnaby/Östersund/Umeå, } \\
\mathrm{SE}^{97}\end{array}$ \\
\hline Skábmagovat & Late January, annual & $1999^{98}$ & Inari, $\mathrm{FI}^{99}$ \\
\hline Riddu Riddu & July, annual & $1991^{100}$ & Kåfjord, NO \\
\hline $\begin{array}{l}\text { Sámi Film } \\
\text { Festival }\end{array}$ & Springtime, annual & $1971^{103}$ & Kautokeino, NO \\
\hline
\end{tabular}

93 "Tampere University of Applied Sciences", Tampere University of Applied Sciences, accessed May 92017 , http://www.tamk.fi/web/tamken/home.

94 "Luleå University of Technology", Luleå University of Technology, accessed May 9 2017, https://www.ltu.se/?l=en.

${ }^{95}$ With the exception of the Sámi Easter festival, all festivals on this list are open to Indigenous filmmakers or artists worldwide, but show a large number of Sámi films due to their location.

${ }_{97}^{96}$ Oskar Östergren in Skype discussion with the author, February 20, 2017.

${ }^{97}$ Ibid.

98 "Skábmagovat Film Festival History", Skábmagovat.fi, accessed May 9 2017,

http://www.Skábmagovat.fi/Skábma_vanhat/Skábma_2010/historia_en.htm.

${ }^{99}$ Ibid.

100 "The History of Riddu Riddu", Riddu Riddu, accessed May 9 2017, http://riddu.no/en/about-riddu-riddufestivala/history-riddu-riddu.

${ }^{101}$ Ibid.

${ }^{102}$ Part of the Sámi Easter Festival. 
Winners of the Skábmagovat Prize

\begin{tabular}{|l|l|l|}
\hline Name $^{104}$ & Profession & Year \\
\hline Katja Gauriloff & Filmmaker & 2017 \\
\hline Johs. Kalvemo & Filmmaker, Journalist & \\
\hline Nils Gaup & Filmmaker & \\
\hline Sverre Porsanger & Actor & \\
\hline Liisa Holmberg & Producer & \\
\hline Oula Näkkäläjärvi & Actor, Writer & \\
\hline Inger-Marie Aikio & Poet, Journalist & \\
\hline
\end{tabular}

Sámi Presence at External Film Festivals and Award Ceremonies

\begin{tabular}{|c|c|c|c|c|c|}
\hline Event & Film & $\begin{array}{l}\text { Festival } \\
\text { specialization? }\end{array}$ & Year & Location & $\begin{array}{l}\text { Awards or } \\
\text { Honours }\end{array}$ \\
\hline $\begin{array}{l}\text { Toronto } \\
\text { International } \\
\text { Film Festival }\end{array}$ & Sameblod $^{105}$ & & 2016 & Toronto, CA & \\
\hline $\begin{array}{l}\text { Toronto } \\
\text { International } \\
\text { Film Festival }\end{array}$ & $\begin{array}{l}\text { Tundra of } \\
\text { Arvas }\end{array}$ & & 2010 & Toronto, CA & $\begin{array}{l}\text { In Competition, } \\
\text { Films from the } \\
\text { North }^{106}\end{array}$ \\
\hline
\end{tabular}

103 "Doing it the Sàmi Way: Beassasmarkanat in Norway", The Nordic Page, last updated January 5, 2017, http://www.tnp.no/norway/exclusive/4466-doing-it-the-Sàmi-way-beassasmarkanat-in-norway.

104 "Skábmagovat Prize to Katja Gauriloff", Skábmagovat.fi, last updated January 28, 2017, accessed May 92017, http://Skábmagovat.fi/Skábmagovat_2014/?page_id=10546.

105"Sámi Blood", Toronto International Film Festival, accessed May 9 2017, http://www.tiff.net/films/Sámi-blood/. 106 "The Tundra of Árvas", LittleBigProductions, accessed May 9 2017, http://littlebig.se/films/the-tundra-of-arvas/. 


\begin{tabular}{|c|c|c|c|c|}
\hline $\begin{array}{l}\text { Venice Film } \\
\text { Festival } \\
\text { (Venice Days } \\
\text { division) }\end{array}$ & Sameblod & 2016 & Venice, IT & $\begin{array}{l}\text { Europa Cinemas } \\
\text { Label (Best } \\
\text { Film, Venice } \\
\text { Days) }{ }^{107} \text {; } \\
\text { Fedeora Award } \\
\text { for Best Young } \\
\text { Director, } \\
\text { Amanda } \\
\text { Kernell }^{108}\end{array}$ \\
\hline $\begin{array}{l}\text { Thessaloniki } \\
\text { Film Festival }\end{array}$ & Sameblod & 2016 & $\begin{array}{l}\text { Thessaloniki, } \\
\text { GR }\end{array}$ & $\begin{array}{l}\text { Human Values } \\
\text { Award }^{109}\end{array}$ \\
\hline $\begin{array}{l}\text { Tokyo } \\
\text { International } \\
\text { Film Festival }\end{array}$ & Sameblod & 2016 & Tokyo, JP & $\begin{array}{l}\text { 2nd place - } \\
\text { Juried } \\
\text { Competition; } \\
\text { Best Actress, } \\
\text { Lene Cecilia } \\
\text { Sparrok }^{110}\end{array}$ \\
\hline
\end{tabular}

\footnotetext{
107 "Amanda Kernell's SÁMI BLOOD (Sameblod) Wins Europa Cinemas Venice Label", Europa Cinemas, last updated September 9, 2016, http://www.europa-cinemas.org/en/News/Activities/Amanda-Kernell-s-SÁMI-BLOODSameblod-wins-Europa-Cinemas-Venice-Label.

108 "Fedeora Awards in 2016", Fedeora (Federation of Film Critics of Europe and the Mediterranean), viewed May 92017 , http://www.fedeora.eu/.

109 "57th Thessaloniki International Film Festival Closing Ceremony", Thessaloniki International Film Festival, accessed May 9 2017, http://www.filmfestival.gr/default.aspx?lang=en-US\&page=607\&newsid=2381.

110 "The 29th Tokyo International Film Festival Award Winners", Tokyo International Film Festival, viewed May 9 2017, http://2016.tiff-jp.net/en/tiff/list_of_winners.html.
} 


\begin{tabular}{|c|c|c|c|c|c|}
\hline $\begin{array}{l}\text { Sundance } \\
\text { Film } \\
\text { Festival }^{111}\end{array}$ & Sameblod & & 2017 & $\begin{array}{l}\text { Park City, } \\
\text { USA }\end{array}$ & $\begin{array}{l}\text { Official } \\
\text { selection, } \\
\text { Spotlight } \\
\text { Program }\end{array}$ \\
\hline $\begin{array}{l}\text { Gothenburg } \\
\text { Film Festival }\end{array}$ & Sameblod & & 2017 & $\begin{array}{l}\text { Gothenburg, } \\
\text { SE }\end{array}$ & $\begin{array}{l}\text { Dragon Award - } \\
\text { Best Nordic } \\
\text { Film; Sven } \\
\text { Nykvist } \\
\text { Cinematography } \\
\text { Award - Sophia } \\
\text { Olsson }{ }^{112}\end{array}$ \\
\hline $\begin{array}{l}\text { Santa Barbara } \\
\text { International } \\
\text { Film Festival }\end{array}$ & Sameblod & & 2017 & $\begin{array}{l}\text { Santa } \\
\text { Barbara, USA }\end{array}$ & $\begin{array}{l}\text { Valhalla Award } \\
\text { - Best Nordic } \\
\text { Film }^{113}\end{array}$ \\
\hline $\begin{array}{l}\text { Sundance } \\
\text { Film } \\
\text { Festival }^{114}\end{array}$ & Stoerre Vaerrie & $\begin{array}{l}\text { Independent } \\
\text { Film }\end{array}$ & 2015 & $\begin{array}{l}\text { Park City, } \\
\text { USA }\end{array}$ & \\
\hline ImagineNative & $\begin{array}{l}\text { Tundra of } \\
\text { Arvas }\end{array}$ & $\begin{array}{l}\text { Indigenous } \\
\text { Film }\end{array}$ & 2010 & Toronto, CA & $\begin{array}{l}\text { Best Music } \\
\text { Video - }\end{array}$ \\
\hline
\end{tabular}

111 Alexander Ortega, "Sundance Review: Sámi Blood", Slug Magazine, last updated January 18, 2017, http://www.slugmag.com/movie-reviews/sundance-film-review-Sámi-blood/.

${ }^{112}$ Alissa Simons, "'Sámi Blood' Tops 40th Goteborg Film Festival", Variety, last updated February 4th, 2017, http://variety.com/2017/film/news/Sámi-blood-2017-40th-goteborg-film-fest-1201978308.

${ }^{113}$ Sarah Ahern, "Santa Barbara Film Festival Announces 2017 Award Winners", Variety, last updated February 11, 2017, http://variety.com/2017/film/news/santa-barbara-film-festival-2017-award-winners-1201984675.

114 "Stoerre Vaerie (Northern Great Mountain) Accepted for Sundance 2015!", Bautafilm, accessed August 2, 2017, http://bautafilm.se/nyheter/stoerre-vaerie-norra-storfjallet-antagen-till-sundance-2015-och-nominerad-forstartsladden-2015/?lang=en. 


\begin{tabular}{|c|c|c|c|c|c|}
\hline & & & & & $\begin{array}{l}\text { Honorable } \\
\text { Mention }^{115}\end{array}$ \\
\hline $\begin{array}{l}\text { Ethnographic } \\
\text { Film Festival }\end{array}$ & $\begin{array}{l}\text { A Shout Into } \\
\text { the Wind }\end{array}$ & & 2008 & Belgrade, RS & $\begin{array}{l}\text { Grand Prix } \\
\text { (Shared) }^{116}\end{array}$ \\
\hline $\begin{array}{l}\text { Arctic Fury } \\
\text { Film Festival }\end{array}$ & The Brooch & Arctic Cinema & 2012 & Rovaniemi, FI & $\begin{array}{l}\text { Honorable } \\
\text { Mention }^{117}\end{array}$ \\
\hline $\begin{array}{l}\text { Tampere Film } \\
\text { Festival }\end{array}$ & $\begin{array}{l}\text { Give Us Our } \\
\text { Skeletons }\end{array}$ & & 1999 & Tampere, FI & $\begin{array}{l}\text { Special } \\
\text { Mention }^{118}\end{array}$ \\
\hline $\begin{array}{l}\text { Lubeck } \\
\text { Nordic Film } \\
\text { Days }\end{array}$ & $\begin{array}{l}\text { Give Us Our } \\
\text { Skeletons }\end{array}$ & & 1999 & Lubeck, DE & $\begin{array}{l}\text { Best } \\
\text { Documentary }{ }^{119}\end{array}$ \\
\hline $\begin{array}{l}\text { Amanda } \\
\text { Awards }\end{array}$ & Ofelas & & 1988 & $\begin{array}{l}\text { Haugesund, } \\
\mathrm{NO}^{120}\end{array}$ & Best Film $^{121}$ \\
\hline $\begin{array}{l}\text { Finnish } \\
\text { Critics' } \\
\text { Association }\end{array}$ & Non Profit & & 2007 & & Annual Prize $^{122}$ \\
\hline Festival de & Ofelas & & 1988 & Sitges, ES & Special \\
\hline
\end{tabular}

115 "The Tundra of Árvas", LittleBigProductions, accessed May 9 2017, http://littlebig.se/films/the-tundra-of-arvas.

116 "A Shout Into the Wind", Sámi Film Database, accessed May 9 2017,

http://www.Skábmagovat.fi/Sámifilm/movies/movie.php?id=101.

117 "The Brooch", Sámi Film Database, accessed May 9 2017,

http://www.Skábmagovat.fi/Sámifilm/movies/movie.php?id=307 .

118 "Give Us Our Skeletons", Sámi Film Database, accessed May 9 2017,

http://www.Skábmagovat.fi/Sámifilm/movies/movie.php?id=60.

${ }^{119}$ Ibid.

120 "Amanda", Norwegian Internationa Film Festival, accessed June 20 2017, https://filmfestivalen.no/en/amanda-3.

121 "Ofelas", Sámi Film Database, accessed June 202017,

http://www.skabmagovat.fi/samifilm/movies/movie.php?id=70.

122 "Non Profit", Sámi Film Database, accessed May 92017,

http://www.Skábmagovat.fi/Sámifilm/movies/movie.php?id=85. 


\begin{tabular}{|c|c|c|c|c|}
\hline Cine de Sitges & & & & Mention $^{123}$ \\
\hline $\begin{array}{l}\text { Academy } \\
\text { Awards }\end{array}$ & Ofelas & 1987 & $\begin{array}{l}\text { Los Angeles, } \\
\text { US }\end{array}$ & $\begin{array}{l}\text { Nomination, } \\
\text { Best Foreign } \\
\text { Language } \\
\text { Film }^{124}\end{array}$ \\
\hline $\begin{array}{l}\text { British Film } \\
\text { Institute }\end{array}$ & Ofelas & 1989 & & $\begin{array}{l}\text { Sutherland } \\
\text { Trophy }\end{array}$ \\
\hline $\begin{array}{l}\text { Yubari } \\
\text { International } \\
\text { Fantastic Film } \\
\text { Festival }\end{array}$ & Ofelas & 1990 & Yubari, JP & $\begin{array}{l}\text { Grand Jury } \\
\text { Prize }^{126}\end{array}$ \\
\hline $\begin{array}{l}\text { Finnish } \\
\text { Quality } \\
\text { Support in } \\
\text { Cinema } \\
\text { Production }\end{array}$ & $\begin{array}{l}\text { Skierri Land of } \\
\text { the Dwarf } \\
\text { Birches }^{127}\end{array}$ & 1982 & & \\
\hline Jussi Awards & $\begin{array}{l}\text { Skierri Land of } \\
\text { the Dwarf }\end{array}$ & 1983 & & $\begin{array}{l}\text { Best Sound } \\
\text { Design }\end{array}$ \\
\hline
\end{tabular}

123 "Ofelas", Sámi Film Database, accessed June 20 2017,

http://www.skabmagovat.fi/samifilm/movies/movie.php?id=70.

${ }_{124}$ Academy Awards Database", Academy of Motion Picture Arts and Sciences, accessed May 92017, http://awardsdatabase.oscars.org/search/results.

125 "Ofelas", Sámi Film Database, accessed June 20 2017,

http://www.skabmagovat.fi/samifilm/movies/movie.php?id=70.

126 "Ofelas", Sámi Film Database, accessed June 202017,

http://www.skabmagovat.fi/samifilm/movies/movie.php?id=70.

127 "Skierri Land of the Dwarf Birches", Sámi Film Database, accessed May 9 2017, http://www.Skábmagovat.fi/Sámifilm/movies/movie.php?id=67. ${ }^{128}$ Ibid. 


\begin{tabular}{|c|c|c|c|c|}
\hline & Birches & & & \\
\hline $\begin{array}{l}\text { Swedish } \\
\text { Television } \\
\text { Awards }\end{array}$ & $\begin{array}{l}\text { Skierri Land of } \\
\text { the Dwarf } \\
\text { Birches }\end{array}$ & 1984 & & $\begin{array}{l}\text { Best Freelance } \\
\text { Work }^{129}\end{array}$ \\
\hline $\begin{array}{l}\text { Dieppe } \\
\text { Festival Films } \\
\text { on the Arctic }\end{array}$ & $\begin{array}{l}\text { Skierri Land of } \\
\text { the Dwarf } \\
\text { Birches }\end{array}$ & 1983 & & $\begin{array}{l}\text { Distinction } \\
\text { Award }^{130}\end{array}$ \\
\hline $\begin{array}{l}\text { Earth Vision } \\
\text { Film Festival }\end{array}$ & $\begin{array}{l}\text { Last Yoik in } \\
\text { Saami Forests }\end{array}$ & 2008 & $\begin{array}{l}\text { Santa Cruz, } \\
\text { US }\end{array}$ & $\begin{array}{l}\text { Kathrine Knight } \\
\text { Award }^{131}\end{array}$ \\
\hline $\begin{array}{l}\text { Global } \\
\text { Indigenous } \\
\text { Film Festival }\end{array}$ & $\begin{array}{l}\text { Last Yoik in } \\
\text { Saami Forests }\end{array}$ & 2008 & $\begin{array}{l}\text { Kathmandu, } \\
\text { NP }\end{array}$ & $\begin{array}{l}\text { The best topical } \\
\text { relevance and } \\
\text { interpretation }^{132}\end{array}$ \\
\hline $\begin{array}{l}\text { Pärnu } \\
\text { International } \\
\text { Documentary } \\
\text { and } \\
\text { Ethnographic } \\
\text { Film Festival }\end{array}$ & $\begin{array}{l}\text { Last Yoik in } \\
\text { Saami Forests }\end{array}$ & 2008 & Pärnu, EE & $\begin{array}{l}\text { The best film on } \\
\text { relationship } \\
\text { between man } \\
\text { and changing } \\
\text { nature }^{133}\end{array}$ \\
\hline $\begin{array}{l}\text { Wildlife } \\
\text { Vaasa Film }\end{array}$ & $\begin{array}{l}\text { Last Yoik in } \\
\text { Saami Forests }\end{array}$ & 2008 & Vaasa, FI & $\begin{array}{l}\text { Environmental } \\
\text { Special Award }\end{array}$ \\
\hline
\end{tabular}

${ }^{129}$ Ibid.

${ }^{130}$ Ibid.

131 "Last Yoik in Sámi Forests", The Sámi Film Database, accessed May 92017, http://www.Skábmagovat.fi/Sámifilm/movies/movie.php?id=84.

${ }^{132}$ Ibid.

${ }^{133}$ Ibid. 


\begin{tabular}{|l|l|l|l|l|l|}
\hline Festival & & & & & by West Finland \\
Environmental \\
Centre
\end{tabular}

\footnotetext{
${ }^{134}$ Ibid.

${ }^{135}$ Ibid.

136 "Let's Dance", Sámi Film Database, accessed June 20 2017,

http://www.skabmagovat.fi/samifilm/movies/movie.php?lang=en\&id=16.

${ }^{137}$ Ibid.

${ }^{138}$ Ibid.
} 


\begin{tabular}{|c|c|c|c|c|c|}
\hline Festival & & & & & \\
\hline $\begin{array}{l}\text { Outokummun } \\
\text { Kinokisa } \\
\text { Competition }\end{array}$ & $\begin{array}{l}\text { Sincere } \\
\text { Intentions }\end{array}$ & & 2004 & & $\begin{array}{l}\text { First Prize, Best } \\
\text { Fiction Film } \\
\text { (Shared) }^{140}\end{array}$ \\
\hline $\begin{array}{l}\text { Blue Sea Film } \\
\text { Festival } \\
\text { Competition }\end{array}$ & $\begin{array}{l}\text { Sincere } \\
\text { Intentions }\end{array}$ & $\begin{array}{l}\text { Baltic amateur } \\
\text { film? }\end{array}$ & 2004 & Rauma, FI & $\begin{array}{l}\text { Special } \\
\text { Mention }^{141}\end{array}$ \\
\hline $\begin{array}{l}\text { Journalist of } \\
\text { Finnmark } \\
\text { Award }^{142}\end{array}$ & Copy Pupils & & 2008 & & \\
\hline $\begin{array}{l}\text { Permanent } \\
\text { Forum on } \\
\text { Indigenous } \\
\text { Issues }^{143}\end{array}$ & $\begin{array}{l}\text { Last Yoik in } \\
\text { Sápmi }\end{array}$ & & 2006 & $\begin{array}{l}\text { New York, } \\
\text { USA }\end{array}$ & Premiere \\
\hline $\begin{array}{l}\text { Arctic Fury } \\
\text { Film Festival }\end{array}$ & $\begin{array}{l}\text { Non-Sámi } \\
\text { Women }\end{array}$ & Arctic Cinema & 2009 & Rovaniemi, FI & $\begin{array}{l}\text { Animation Film } \\
\text { Award }^{144}\end{array}$ \\
\hline Jussi Awards & $\begin{array}{l}\text { The Minister of } \\
\text { State }\end{array}$ & & 1998 & & $\begin{array}{l}\text { Costume } \\
\text { Design, Tiina }\end{array}$ \\
\hline
\end{tabular}

${ }^{139}$ Ibid.

${ }^{140}$ Sincere Intention s", Sámi Film Database, accessed May 92017, http://www.Skábmagovat.fi/Sámifilm/movies/movie.php?id=99.

${ }^{141}$ Ibid.

142 "Copy Pupils", Sámi Film Database, accessed June 20 2017,

http://www.skabmagovat.fi/samifilm/movies/movie.php?id=103.

143 "Last Yoik in Sámi Forests", The Sámi Film Database, accessed May 92017,

http://www.Skábmagovat.fi/Sámifilm/movies/movie.php?id=84.

144 "Non-Sámi Women", Sámi Film Database, accessed June 202017,

http://www.skabmagovat.fi/samifilm/movies/movie.php?id=258. 


\begin{tabular}{|c|c|c|c|c|}
\hline & & & & $\begin{array}{l}\text { Kaukanen; Art } \\
\text { Direction, Risto } \\
\text { Karhula (both } \\
\text { shared with film } \\
\text { Lunastus) }^{145}\end{array}$ \\
\hline $\begin{array}{l}\text { Gothenburg } \\
\text { Film Festival }\end{array}$ & Herdswoman $^{146}$ & 2008 & $\begin{array}{l}\text { Gothenburg, } \\
\text { SE }\end{array}$ & \\
\hline $\begin{array}{l}\text { Lubeck } \\
\text { Nordic Film } \\
\text { Days }\end{array}$ & Herdswoman & 2008 & Lubeck, DE & $\begin{array}{l}\text { Best } \\
\text { Documentary }{ }^{147}\end{array}$ \\
\hline
\end{tabular}

Websites Showing Sámi Film

- Youtube.com - largest video sharing site in the world and second most-visited site on the Internet ${ }^{148}$ - headquartered in California, USA, and owned by Google ${ }^{149}$. Used by Sámi artists - some films may be on the site in violation of copyright.

- Vimeo.com - Another large video sharing site, this time focused on creative works, including short films. ${ }^{150}$ Many films on this site are uploaded by the artists themselves, or small film companies, including those by Sámi artists.

\footnotetext{
145 "The Minister of State", Sámi Film Database, accessed June 202017, http://www.skabmagovat.fi/samifilm/movies/movie.php?lang=en\&id=61 .

146 "Herdswoman", Sámi Film Database, accessed May 92017, http://www.Skábmagovat.fi/Sámifilm/movies/movie.php?id=133.

${ }^{147}$ Ibid.

148 "YouTube.com Traffic Statistics", Alexa, accessed May 9 2017, http://www.alexa.com/siteinfo/youtube.com.

${ }^{149}$ Michael Arrington, "Google Has Acquired YouTube", TechCrunch, last updated October 9, 2006, https://techcrunch.com/2006/10/09/google-has-acquired-youtube.

150 "About Vimeo", Vimeo, accessed May 9 2017, https://vimeo.com/about.
} 
- Nuorajtv.no - Based in Norway. Lule Sámi website created by Sámi youth (founder Lars Theodor Kintel) to offer Lule-language media for young people ${ }^{151}$. Funded by the Sámi Parliament, Nordland county and Ministry of Government Administration, Reform and Church Affairs ${ }^{152}$.

- Isuma.tv - Multimedia online platform for Indigenous artists worldwide. Based in Montreal, Canada, and driven by Canada's Inuit community. Contains multiple Sámi "communities" containing videos by or about Sámi people. ${ }^{153}$

- Samediggi.fi - Website of the Sámi Parliament in Inari, FI. Showcases student media projects, including film, video, audio, and photography. Links from Sámi Film Database are mostly dead. ${ }^{154}$

- $\quad$ sogku.fi - Website of the Sámi Education Centre in Inari, FI. Features films produced by Indigenous Film Skabma, the regional centre for filmmaking in Inari - however, most links to video (from Sámi Film Database) appear to be dead. ${ }^{155}$

\footnotetext{
151"Om NuorajTV", nuoraj.tv, accessed June 5 2017, http://nuorajtv.no/om-nuorajtv/. (Norwegian)

152 "About Us", Isuma.tv, accessed May 9 2017, http://www.isuma.tv/about-us.

153 "Search", Isuma.tv, accessed May 9 2017, http://www.isuma.tv/channels/Sámi.

154"Samediggi", Samediggi.fi, accessed June 20 2017, http://www.samediggi.fi.

155 "SOGSAKK Medialinja", sogku.fi, accessed June 20 2017, http://sogku.fi.
} 


\section{Appendix C: List of Sámi Films}

Films Listed on the Sámi Film Database ${ }^{156}$

\begin{tabular}{|c|c|c|c|c|c|c|c|}
\hline Film & $\begin{array}{l}\text { Count } \\
\text { ry }\end{array}$ & Producer & $\begin{array}{l}\text { Type of } \\
\text { Producer }\end{array}$ & Filmmaker & Year & Runtime & Genre \\
\hline $01: 25.3$ & NO & $\begin{array}{l}\text { Nordland } \\
\text { College of } \\
\text { Art and Film }\end{array}$ & Educational & $\begin{array}{l}\text { Marja Bål Nango, } \\
\text { Daniel Bianchini, } \\
\text { Vidar Wikran }\end{array}$ & 2011 & $2 \mathrm{~min}$ & Short \\
\hline $\begin{array}{l}1000 \\
\text { Years }\end{array}$ & FI & $\begin{array}{l}\text { Sámi Siida } \\
\text { Ry }\end{array}$ & Non-profit & $\begin{array}{l}\text { Jussi Isokoski, } \\
\text { Niilo Rasmus, } \\
\text { Utsjoen nuoret }\end{array}$ & 2008 & $4 \min$ & Short \\
\hline 365 Days & $\mathrm{NO}$ & $\begin{array}{l}\text { Finnmark } \\
\text { University } \\
\text { College }\end{array}$ & Educational & $\begin{array}{l}\text { Lemet Ailo } \\
\text { Holmestrand }\end{array}$ & 2011 & $19 \min$ & $\begin{array}{l}\text { Docume } \\
\text { ntary }\end{array}$ \\
\hline $\begin{array}{l}9175 \\
\text { Sveagruva }\end{array}$ & $\mathrm{NO}$ & $\begin{array}{l}\text { Roger } \\
\text { Mandal }\end{array}$ & Individual & Roger Mandal & 2007 & $26 \mathrm{~min}$ & $\begin{array}{l}\text { Docume } \\
\text { ntary }\end{array}$ \\
\hline $\begin{array}{l}\text { A } \\
\text { Childhood } \\
\text { in Prison }\end{array}$ & $\mathrm{NO}$ & NRK & State media & $\begin{array}{l}\text { Johs. Kalvemo, } \\
\text { Tove Austad }\end{array}$ & 2005 & $30 \mathrm{~min}$ & $\begin{array}{l}\text { Docume } \\
\text { ntary }\end{array}$ \\
\hline $\begin{array}{l}\text { A City } \\
\text { Sámi in } \\
\text { the Woods }\end{array}$ & SE & $\begin{array}{l}\text { Liselotte } \\
\text { Wajstedt }\end{array}$ & Individual & Liselotte Wajstedt & 2007 & $\begin{array}{l}4 \min 40 \\
\text { sec }\end{array}$ & Short \\
\hline
\end{tabular}

156"The Sàmi Film Database", Skábmagovat.fi, accessed May 9 2017, http://www.Skábmagovat.fi/Sàmifilm/movies. 


\begin{tabular}{|c|c|c|c|c|c|c|c|}
\hline $\begin{array}{l}\text { A } \\
\text { Dedicated } \\
\text { non-Sámi }\end{array}$ & NO & $\begin{array}{l}\text { Barentsfilm } \\
\text { A/S }\end{array}$ & $\begin{array}{l}\text { Private } \\
\text { company }\end{array}$ & $\begin{array}{l}\text { Anne Kjersti } \\
\text { Björn }\end{array}$ & 1996 & $8 \mathrm{~min}$ & $\begin{array}{l}\text { Docume } \\
\text { ntary }\end{array}$ \\
\hline $\begin{array}{l}\text { A Good } \\
\text { Life in the } \\
\text { North }\end{array}$ & FI & Sogsakk & Educational & Eeva Nykänen & 2009 & $8 \mathrm{~min}$ & Short \\
\hline $\begin{array}{l}\text { A Long } \\
\text { Lesson } \\
\text { Beyond } \\
\text { Lake Inari }\end{array}$ & FI & $\begin{array}{l}\text { Saamelaisalu } \\
\text { een } \\
\text { koulutuskesk } \\
\text { us Sogsakk }\end{array}$ & Educational & Ulpu Siponen & 2012 & $21 \mathrm{~min}$ & Short \\
\hline $\begin{array}{l}\text { A } \\
\text { Migratory } \\
\text { Bird }\end{array}$ & FI & $\begin{array}{l}\text { Saamelaisalu } \\
\text { een } \\
\text { koulutuskesk } \\
\text { us SAKK }\end{array}$ & Educational & Pia Mikkilä & 2011 & $10 \mathrm{~min}$ & $\begin{array}{l}\text { Docume } \\
\text { ntary }\end{array}$ \\
\hline $\begin{array}{l}\text { A } \\
\text { Possibility }\end{array}$ & FI & Sogsakk & Educational & $\begin{array}{l}\text { Anneli } \\
\text { Lappalainen }\end{array}$ & 2009 & $15 \mathrm{~min}$ & Short \\
\hline $\begin{array}{l}\text { A Red } \\
\text { Girls } \\
\text { Reasoning }\end{array}$ & $\mathrm{CA}$ & $\begin{array}{l}\text { Rose } \\
\text { Stiffarm, The } \\
\text { Crazy8s Film } \\
\text { Society }\end{array}$ & $\begin{array}{l}\text { Mixed } \\
\text { (Individual, } \\
\text { non-profit) }\end{array}$ & $\begin{array}{l}\text { Elle-Maija } \\
\text { Tailfeathers }\end{array}$ & 2012 & $10 \mathrm{~min}$ & Short \\
\hline $\begin{array}{l}\text { A Sámi in } \\
\text { the City }\end{array}$ & SE & $\begin{array}{l}\text { Liselotte } \\
\text { Wajstedt }\end{array}$ & Individual & Liselotte Wajstedt & 2007 & $8 \min$ & $\begin{array}{l}\text { Docume } \\
\text { ntary }\end{array}$ \\
\hline A Shout & FI & Oktober Oy & Private & Katja Gauriloff & 2007 & $55 \mathrm{~min}$ & Docume \\
\hline
\end{tabular}




\begin{tabular}{|c|c|c|c|c|c|c|c|}
\hline $\begin{array}{l}\text { Into the } \\
\text { Wind }\end{array}$ & & & company & & & & ntary \\
\hline $\begin{array}{l}\text { A } \\
\text { Smoking } \\
\text { Grandma }\end{array}$ & FI & Sogsakk & Educational & Kirsti Länsman & 2008 & $5 \mathrm{~min}$ & Short \\
\hline $\begin{array}{l}\text { A Son of } \\
\text { Two } \\
\text { Worlds }\end{array}$ & FI & $\begin{array}{l}\text { Ima Filbma- } \\
\text { ja sátneduodji }\end{array}$ & $\begin{array}{l}\text { Private } \\
\text { company }\end{array}$ & $\begin{array}{l}\text { Ima Aikio- } \\
\text { Arianaik }\end{array}$ & 2007 & $27 \mathrm{~min}$ & $\begin{array}{l}\text { Docume } \\
\text { ntary }\end{array}$ \\
\hline $\begin{array}{l}\text { A Sprinkle } \\
\text { of } \\
\text { Laughter }\end{array}$ & FI & $\begin{array}{l}\text { Saamelaisalu } \\
\text { een } \\
\text { koulutuskesk } \\
\text { us SAKK }\end{array}$ & Educational & $\begin{array}{l}\text { Ima Aikio- } \\
\text { Arianaik }\end{array}$ & 2005 & $26 \mathrm{~min}$ & Short \\
\hline $\begin{array}{l}\text { A Summer } \\
\text { Bird in the } \\
\text { Land of } \\
\text { Winter }\end{array}$ & $\mathrm{NO}$ & $\begin{array}{l}\text { NRK, Sámi } \\
\text { Radio }\end{array}$ & State media & $\begin{array}{l}\text { Nils John } \\
\text { Porsanger }\end{array}$ & 2009 & $28 \mathrm{~min}$ & $\begin{array}{l}\text { Docume } \\
\text { ntary }\end{array}$ \\
\hline A Wish & FI & $\begin{array}{l}\text { Saamelaisalu } \\
\text { een } \\
\text { koulutuskesk } \\
\text { us Sogsakk }\end{array}$ & Educational & Onneli Halonen & 2012 & $2 \min$ & Short \\
\hline Aallokosta & FI & Sogsakk & Educational & Marjo Ulkuniemi & 2011 & $2 \min$ & Short \\
\hline $\begin{array}{l}\text { Aanaar } \\
\text { Eennam - }\end{array}$ & FI & $\begin{array}{l}\text { Yleisradio } \\
\text { Oy, SVT }\end{array}$ & State media & Ella Sarre & 1983 & $35 \mathrm{~min}$ & $\begin{array}{l}\text { Docume } \\
\text { ntary }\end{array}$ \\
\hline
\end{tabular}




\begin{tabular}{|c|c|c|c|c|c|c|c|}
\hline $\begin{array}{l}\text { The Land } \\
\text { of Inari }\end{array}$ & & & & & & & \\
\hline $\begin{array}{l}\text { Adventure } \\
\text { at Sea }\end{array}$ & FI & Sogsakk & Educational & Maria Kosonen & 2012 & $\begin{array}{l}1 \min 30 \\
\sec \end{array}$ & Short \\
\hline $\begin{array}{l}\text { Against } \\
\text { the Time } \\
\text { in the } \\
\text { Lyngen } \\
\text { Alps }\end{array}$ & NO & Semferno & $\begin{array}{l}\text { Private } \\
\text { company }\end{array}$ & $\begin{array}{l}\text { Kate Hilde } \\
\text { Nilsen, Bente } \\
\text { Fernando Sem }\end{array}$ & 2008 & $62 \mathrm{~min}$ & $\begin{array}{l}\text { Docume } \\
\text { ntary }\end{array}$ \\
\hline $\begin{array}{l}\text { Agent } \\
\text { Hahkaoajv } \\
\text { ve }\end{array}$ & NO & Nuoraj TV & $\begin{array}{l}\text { Mixed } \\
\text { (multiple } \\
\text { funding) }\end{array}$ & $\begin{array}{l}\text { Simon Piera } \\
\text { Paulsen }\end{array}$ & 2010 & $2 \min$ & Short \\
\hline $\begin{array}{l}\text { Ailo Sets } \\
\text { Out North }\end{array}$ & NO & $\begin{array}{l}\text { The Nordic } \\
\text { Sámi } \\
\text { Institute/Sám } \\
\text { i Instituhtta }\end{array}$ & Educational & Solveig Joks & 2007 & $38 \mathrm{~min}$ & Short \\
\hline $\begin{array}{l}\text { Ajggie - } \\
\text { Time }\end{array}$ & SE & Indigee & Non-profit & Liselotte Wajstedt & 2013 & $8 \min$ & Short \\
\hline $\begin{array}{l}\text { Almost } \\
\text { Like a } \\
\text { Winter } \\
\text { Dream }\end{array}$ & FI & Sogsakk & Educational & Marianne Aikio & 2010 & $7 \mathrm{~min}$ & Short \\
\hline An & FI & Saamelaisalu & Educational & Irene Länsman & 2011 & $13 \mathrm{~min}$ & Short \\
\hline
\end{tabular}




\begin{tabular}{|c|c|c|c|c|c|c|c|}
\hline $\begin{array}{l}\text { Outsider } \\
\text { at Home }\end{array}$ & & $\begin{array}{l}\text { een } \\
\text { koulutuskesk } \\
\text { us SAKK }\end{array}$ & & & & & \\
\hline Ancestors & FI & $\begin{array}{l}\text { Saamelaisalu } \\
\text { een } \\
\text { koulutuskesk } \\
\text { us Sogsakk }\end{array}$ & Educational & Sunna Kitti & 2012 & $6 \mathrm{~min}$ & Short \\
\hline $\begin{array}{l}\text { Ancient } \\
\text { Forces }\end{array}$ & NO & & Unknown & $\begin{array}{l}\text { Niels Ovlla } \\
\text { Dunfjell }\end{array}$ & 2011 & $3 \min$ & Short \\
\hline $\begin{array}{l}\text { And in the } \\
\text { winter we } \\
\text { live in iglu }\end{array}$ & FI & Samediggi & Non-profit & Milla Pulska & 2006 & $1 \mathrm{~min}$ & Short \\
\hline $\begin{array}{l}\text { And the } \\
\text { Mountains } \\
\text { are the } \\
\text { Same }\end{array}$ & $\mathrm{NO}$ & Gjert Rognli & Individual & Gjert Rognli & 2006 & $6 \mathrm{~min}$ & Short \\
\hline $\begin{array}{l}\text { And Then } \\
\text { Came the } \\
\text { Flood }\end{array}$ & $\begin{array}{l}\text { FI/SE/ } \\
\text { NO }\end{array}$ & $\begin{array}{l}\text { Filmpool } \\
\text { Nord, } \\
\text { NRK/Sámi } \\
\text { Radio, SVT, } \\
\text { YLE, NRK, } \\
\text { Filbmagoahti } \\
\text { AS, Zentropa }\end{array}$ & $\begin{array}{l}\text { Mixed } \\
\text { (State } \\
\text { media, } \\
\text { educational, } \\
\text { private } \\
\text { company) }\end{array}$ & $\begin{array}{l}\text { Kalle Mannela, } \\
\text { Johs. Kalvemo, } \\
\text { Per Niia }\end{array}$ & 1976 & $35 \mathrm{~min}$ & $\begin{array}{l}\text { Docume } \\
\text { ntary }\end{array}$ \\
\hline
\end{tabular}




\begin{tabular}{|c|c|c|c|c|c|c|c|}
\hline & & $\begin{array}{l}\text { Entertainmen } \\
\text { ts7 ApS, } \\
\text { Sogsakk, } \\
\text { Nordland } \\
\text { College of } \\
\text { Art and Film, } \\
\text { Film Form } \\
\text { AB }\end{array}$ & & & & & \\
\hline $\begin{array}{l}\text { and, the } \\
\text { movement } \\
\text { from the } \\
\text { past }\end{array}$ & NO & Daban Dan & Unknown & $\begin{array}{l}\text { Gjert Rognli, } \\
\text { Asbjörn Forsöget }\end{array}$ & 2006 & $9 \min$ & Short \\
\hline $\begin{array}{l}\text { Anders } \\
\text { Forsdahl's } \\
\text { Curve }\end{array}$ & $\mathrm{NO}$ & $\begin{array}{l}\text { Ellen-Astri } \\
\text { Lundby, } \\
\text { University of } \\
\text { Troms } \varnothing\end{array}$ & $\begin{array}{l}\text { Mixed } \\
\text { (individual, } \\
\text { educational) }\end{array}$ & $\begin{array}{l}\text { Ellen-Astri } \\
\text { Lundby }\end{array}$ & 2011 & $15 \mathrm{~min}$ & $\begin{array}{l}\text { Docume } \\
\text { ntary }\end{array}$ \\
\hline $\begin{array}{l}\text { Animated } \\
\text { Ants }\end{array}$ & FI & Sogsakk & Educational & Jouni West & 2010 & $3 \min$ & Short \\
\hline $\begin{array}{l}\text { Appearanc } \\
\text { e and } \\
\text { Disappear } \\
\text { ance }\end{array}$ & FI & $\begin{array}{l}\text { Saamelaisalu } \\
\text { een } \\
\text { koulutuskesk } \\
\text { us Sogsakk }\end{array}$ & Educational & Susanna Rauno & 2013 & $20 \mathrm{~min}$ & Short \\
\hline Art From & $\mathrm{NO} / \mathrm{FI}$ & NRK/Sámi & State media & Jaana & 2002 & $30 \mathrm{~min}$ & Docume \\
\hline
\end{tabular}




\begin{tabular}{|c|c|c|c|c|c|c|c|}
\hline the Heart & & Radio & & $\begin{array}{l}\text { Niittyvuopio, } \\
\text { John Erling Utsi }\end{array}$ & & & ntary \\
\hline $\begin{array}{l}\text { Art in the } \\
\text { Forestland }\end{array}$ & $\mathrm{SE}$ & $\begin{array}{l}\text { Hans-Olov } \\
\text { Utsi AB }\end{array}$ & $\begin{array}{l}\text { Private } \\
\text { Company }\end{array}$ & $\begin{array}{l}\text { Ann-Christine } \\
\text { Haupt }\end{array}$ & 2007 & $28 \min$ & $\begin{array}{l}\text { Docume } \\
\text { ntary }\end{array}$ \\
\hline $\begin{array}{l}\text { Bahcan } \\
\text { guohtoeat } \\
\text { namat }\end{array}$ & $\mathrm{NO}$ & $\begin{array}{l}\text { NRK/Sámi } \\
\text { Radio }\end{array}$ & State Media & Thoralf Balto & 2004 & $30 \mathrm{~min}$ & $\begin{array}{l}\text { Docume } \\
\text { ntary }\end{array}$ \\
\hline Baptizing & $\begin{array}{l}\text { NO } \\
\text { (Sápm } \\
\text { i) }\end{array}$ & $\begin{array}{l}\text { NRK/Sámi } \\
\text { Radio }\end{array}$ & State Media & Eilif Aslaksen & 2004 & $5 \mathrm{~min}$ & $\begin{array}{l}\text { Docume } \\
\text { ntary }\end{array}$ \\
\hline $\begin{array}{l}\text { Barents } \\
\text { Fashion } \\
\text { Week }\end{array}$ & $\mathrm{NO}$ & $\begin{array}{l}\text { Kautokeino } \\
\text { Film AS }\end{array}$ & $\begin{array}{l}\text { Private } \\
\text { Company }\end{array}$ & Nils Gaup & 2008 & $55 \mathrm{~min}$ & $\begin{array}{l}\text { Docume } \\
\text { ntary }\end{array}$ \\
\hline Bazo & $\mathrm{NO}$ & $\begin{array}{l}\text { Filmpool } \\
\text { Nord, } \\
\text { Filbmagoahti } \\
\text { AS, Zentropa } \\
\text { Entertainmen } \\
\text { ts7 ApS, } \\
\text { Nordland } \\
\text { College of } \\
\text { Art and Film, } \\
\text { Film Form }\end{array}$ & $\begin{array}{l}\text { Mixed } \\
\text { (Private } \\
\text { Company, } \\
\text { Educational } \\
\text { ) }\end{array}$ & $\begin{array}{l}\text { Lars Göran } \\
\text { Pettersson }\end{array}$ & 2003 & $\begin{array}{l}1 \mathrm{hr} 32 \\
\min \end{array}$ & Feature \\
\hline
\end{tabular}




\begin{tabular}{|c|c|c|c|c|c|c|c|}
\hline & & $\mathrm{AB}$ & & & & & \\
\hline BBB & FI & & Unknown & $\begin{array}{l}\text { Ima Aikio- } \\
\text { Arianaick }\end{array}$ & 2013 & $3 \mathrm{~min}$ & Short \\
\hline $\begin{array}{l}\text { Before } \\
\text { She Came, } \\
\text { After He } \\
\text { Left }\end{array}$ & $\mathrm{NO}$ & $\begin{array}{l}\text { Nordland } \\
\text { College of } \\
\text { Art and Film }\end{array}$ & Educational & Marja Bål Nango & 2011 & $25 \mathrm{~min}$ & $\begin{array}{l}\text { Docume } \\
\text { ntary }\end{array}$ \\
\hline $\begin{array}{l}\text { Before } \\
\text { She Came, } \\
\text { After He } \\
\text { Left }\end{array}$ & $\mathrm{NO}$ & $\begin{array}{l}\text { Nordland } \\
\text { College of } \\
\text { Art and Film }\end{array}$ & Educational & Marja Bål Nango & 2012 & $22 \mathrm{~min}$ & Short \\
\hline $\begin{array}{l}\text { Behind the } \\
\text { Silverwidt } \\
\text { hs }\end{array}$ & $\mathrm{NO}$ & & Unknown & Gjert Rognli & 2005 & $4 \min$ & Short \\
\hline $\begin{array}{l}\text { Between } \\
\text { Two } \\
\text { Realities }\end{array}$ & FI & Sogsakk & Educational & Erika Satta & 2007 & $6 \mathrm{~min}$ & $\begin{array}{l}\text { Docume } \\
\text { ntary }\end{array}$ \\
\hline $\begin{array}{l}\text { Beyond } \\
\text { Day and } \\
\text { Night }\end{array}$ & $\begin{array}{l}\text { FI/NO } \\
/ \mathrm{SE}\end{array}$ & $\begin{array}{l}\text { Nordfilm Oy, } \\
\text { PAS Film } \\
\text { AB, } \\
\text { Yleisradio } \\
\text { Oy, SVT, } \\
\text { SVT } 2 \text { Luleå, }\end{array}$ & $\begin{array}{l}\text { Mixed } \\
\text { (State } \\
\text { media, } \\
\text { private } \\
\text { company) }\end{array}$ & $\begin{array}{l}\text { Paul-Anders } \\
\text { Simma }\end{array}$ & 1988 & $49 \mathrm{~min}$ & $\begin{array}{l}\text { Children' } \\
\text { s Film }\end{array}$ \\
\hline
\end{tabular}




\begin{tabular}{|c|c|c|c|c|c|c|c|}
\hline & & $\begin{array}{l}\text { Nordnorsk } \\
\text { filmsenter, } \\
\text { Statens } \\
\text { filmcentral, } \\
\text { Giron-filmi } \\
\text { Oy, Suomen } \\
\text { elokuvasäätiö } \\
\text { SES, SFI } \\
\text { Svenska } \\
\text { Filminstitutet }\end{array}$ & & & & & \\
\hline Bloodland & $\mathrm{CA}$ & $\begin{array}{l}\text { Elle-Maija } \\
\text { Tailfeathers }\end{array}$ & & $\begin{array}{l}\text { Elle-Maija } \\
\text { Tailfeathers }\end{array}$ & 2011 & $4 \min$ & Short \\
\hline $\begin{array}{l}\text { Brothers } \\
\text { in Kiruna }\end{array}$ & SE & SVT Sápmi & State media & Per Niia & 2004 & $28 \mathrm{~min}$ & Short \\
\hline $\begin{array}{l}\text { Building a } \\
\text { Turf Hut } \\
\text { in the } \\
\text { Traditiona } \\
1 \text { Way }\end{array}$ & $\mathrm{NO}$ & $\begin{array}{l}\text { RiddoDuottar } \\
\text { Museat }\end{array}$ & Non-profit & Solveig Joks & 2010 & $10 \mathrm{~min}$ & Short \\
\hline $\begin{array}{l}\text { Butt- } \\
\text { Niillas }\end{array}$ & $\mathrm{NO}$ & $\begin{array}{l}\text { Per-Josef } \\
\text { Idivuoma }\end{array}$ & Individual & $\begin{array}{l}\text { Per-Josef } \\
\text { Idivuoma }\end{array}$ & 2009 & $2 \min$ & Short \\
\hline $\begin{array}{l}\text { Canned } \\
\text { Dreams }\end{array}$ & FI & Oktober Oy & $\begin{array}{l}\text { Private } \\
\text { Company }\end{array}$ & Katja Gauriloff & 2012 & $78 \mathrm{~min}$ & $\begin{array}{l}\text { Docume } \\
\text { ntary }\end{array}$ \\
\hline
\end{tabular}




\begin{tabular}{|c|c|c|c|c|c|c|c|}
\hline $\begin{array}{l}\text { Carried } \\
\text { By the } \\
\text { Stream }\end{array}$ & FI & $\begin{array}{l}\text { Ima Filbma- } \\
\text { ja sátneduodji }\end{array}$ & $\begin{array}{l}\text { Private } \\
\text { Company }\end{array}$ & $\begin{array}{l}\text { Ima Aikio- } \\
\text { Arianaick }\end{array}$ & 2013 & $3 \min$ & Short \\
\hline $\begin{array}{l}\text { Citizens } \\
\text { Without } \\
\text { Rights }\end{array}$ & FI & $\begin{array}{l}\text { Yleisradio } \\
\text { FST } \\
\text { Asiaohjelmat }\end{array}$ & State media & Agot Jung & 1971 & $50 \mathrm{~min}$ & $\begin{array}{l}\text { Docume } \\
\text { ntary }\end{array}$ \\
\hline $\begin{array}{l}\text { Citroen } \\
\text { Trip }\end{array}$ & $\mathrm{NO}$ & $\begin{array}{l}\text { NRK/Sámi } \\
\text { Radio }\end{array}$ & State media & Johs. Kalvemo & 2001 & $30 \mathrm{~min}$ & Short \\
\hline Cockoo & $\mathrm{NO}$ & $\begin{array}{l}\text { Linda Overli } \\
\text { Nilsen }\end{array}$ & Individual & Ken Are Bongo & 2014 & $6 \mathrm{~min}$ & Short \\
\hline $\begin{array}{l}\text { Copy } \\
\text { Pupils }\end{array}$ & $\mathrm{NO}$ & $\begin{array}{l}\text { NRK/Sámi } \\
\text { Radio }\end{array}$ & State media & $\begin{array}{l}\text { Jan Henrik } \\
\text { Heatta, Maret } \\
\text { Elin Kemi }\end{array}$ & 2008 & $20 \mathrm{~min}$ & $\begin{array}{l}\text { TV } \\
\text { Producti } \\
\text { on }\end{array}$ \\
\hline $\begin{array}{l}\text { Countless } \\
\text { Strokes on } \\
\text { the Teno } \\
\text { River }\end{array}$ & $\begin{array}{l}\text { NO } \\
\text { (Sápm } \\
\text { i) }\end{array}$ & $\begin{array}{l}\text { NRK/Sámi } \\
\text { Radio }\end{array}$ & State media & Thoralf Balto & 2005 & $28 \mathrm{~min}$ & $\begin{array}{l}\text { Docume } \\
\text { ntary }\end{array}$ \\
\hline $\begin{array}{l}\text { Crafted } \\
\text { By the } \\
\text { Past }\end{array}$ & FI & $\begin{array}{l}\text { Saamelaisalu } \\
\text { een } \\
\text { koulutuskesk } \\
\text { us SAKK }\end{array}$ & Educational & $\begin{array}{l}\text { Vilma } \\
\text { Rautakangas }\end{array}$ & 2011 & $18 \mathrm{~min}$ & $\begin{array}{l}\text { Docume } \\
\text { ntary }\end{array}$ \\
\hline Cujaju & $\mathrm{NO}$ & $\begin{array}{l}\text { Sonar } \\
\text { Film/Britt }\end{array}$ & $\begin{array}{l}\text { Mixed } \\
\text { (individual, }\end{array}$ & Gustav Kvaal & 2010 & $3 \min$ & Short \\
\hline
\end{tabular}




\begin{tabular}{|c|c|c|c|c|c|c|c|}
\hline & & Kramvig & $\begin{array}{l}\text { private } \\
\text { company) }\end{array}$ & & & & \\
\hline $\begin{array}{l}\text { Dancing } \\
\text { Virgin }\end{array}$ & $\mathrm{NO}$ & Siivet AS & $\begin{array}{l}\text { Private } \\
\text { company }\end{array}$ & $\begin{array}{l}\text { Anstein } \\
\text { Mikkelsen }\end{array}$ & 2010 & $14 \min$ & Short \\
\hline $\begin{array}{l}\text { Darned } \\
\text { Lapp }\end{array}$ & SE & IdiStudios & $\begin{array}{l}\text { Private } \\
\text { company }\end{array}$ & $\begin{array}{l}\text { Per-Josef } \\
\text { Idivuoma }\end{array}$ & 2010 & $3 \min$ & Short \\
\hline $\begin{array}{l}\text { Dead } \\
\text { Soon } \\
\text { After } \\
\text { Birth }\end{array}$ & NO & $\begin{array}{l}\text { Kautokeino } \\
\text { Film AS }\end{array}$ & $\begin{array}{l}\text { Private } \\
\text { company }\end{array}$ & $\begin{array}{l}\text { Anne Merete } \\
\text { Gaup }\end{array}$ & 2011 & $7 \min$ & Short \\
\hline Debt & FI & Sogsakk & Educational & Henri Portti & 2011 & $2 \min$ & Short \\
\hline $\begin{array}{l}\text { Denied } \\
\text { Education } \\
\text { in the Lule } \\
\text { Sámi } \\
\text { Language }\end{array}$ & $\mathrm{NO}$ & Nuoraj TV & Mixed & Maria Mikkelsen & 2010 & $4 \min$ & Short \\
\hline $\begin{array}{l}\text { Det nya } \\
\text { vädrets } \\
\text { offer }\end{array}$ & SE & SVT Sápmi & $\begin{array}{l}\text { Private } \\
\text { company }\end{array}$ & Mariela Idivuoma & 2008 & $28 \mathrm{~min}$ & $\begin{array}{l}\text { TV } \\
\text { Producti } \\
\text { on }\end{array}$ \\
\hline $\begin{array}{l}\text { Different } \\
\text { Kind of } \\
\text { Reindeer } \\
\text { Herder }\end{array}$ & FI & Sogsakk & Educational & Janne Kyrö & 2010 & $2 \min$ & Short \\
\hline
\end{tabular}




\begin{tabular}{|c|c|c|c|c|c|c|c|}
\hline Distance & FI & Sogsakk & Educational & Pia Mikkilä & 2010 & $1 \mathrm{~min}$ & Short \\
\hline Dog's Life & $\mathrm{FI} / \mathrm{FR}$ & $\begin{array}{l}\text { Telegoahti } \\
\text { O/S, PAS } \\
\text { Film AB, AB } \\
\text { Saamifilm } \\
\text { Oy }\end{array}$ & $\begin{array}{l}\text { Private } \\
\text { Company }\end{array}$ & $\begin{array}{l}\text { Paul-Anders } \\
\text { Simma, Erling } \\
\text { Söderström }\end{array}$ & 1996 & $\begin{array}{l}24 \mathrm{~min} \\
59 \mathrm{sec}\end{array}$ & Short \\
\hline $\begin{array}{l}\text { Down and } \\
\text { Out }\end{array}$ & FI & $\begin{array}{l}\text { Saamelaisalu } \\
\text { een } \\
\text { koulutuskesk } \\
\text { us SAKK }\end{array}$ & Educational & Virva Guttorm & 2005 & $17 \mathrm{~min}$ & $\begin{array}{l}\text { Docume } \\
\text { ntary }\end{array}$ \\
\hline $\begin{array}{l}\text { Duoggi- } \\
\text { donto } \\
\text { gavdnosat }\end{array}$ & FI & Sogsakk & Educational & Onneli Halonen & 2011 & $\begin{array}{l}1 \min 20 \\
\sec \end{array}$ & Short \\
\hline $\begin{array}{l}\text { Duottareai } \\
\text { su - A } \\
\text { Trip to the } \\
\text { Fell } \\
\text { Country }\end{array}$ & FI & $\begin{array}{l}\text { Saamelaisalu } \\
\text { een } \\
\text { koulutuskesk } \\
\text { us SAKK }\end{array}$ & Educational & Oula Järvensivu & 2006 & $1 \mathrm{~min}$ & $\begin{array}{l}\text { Children' } \\
\text { s Film }\end{array}$ \\
\hline $\begin{array}{l}\text { Eallima } \\
\text { ravddas }\end{array}$ & $\mathrm{NO}$ & $\begin{array}{l}\text { NRK/Sámi } \\
\text { Radio }\end{array}$ & State Media & Suvi West & 2006 & $30 \mathrm{~min}$ & $\begin{array}{l}\text { Docume } \\
\text { ntary }\end{array}$ \\
\hline $\begin{array}{l}\text { Earth of } \\
\text { Rage }\end{array}$ & $\mathrm{FI} / \mathrm{NO}$ & $\begin{array}{l}\text { Telegoahti } \\
\text { O/S, PAS } \\
\text { Film AB, AB }\end{array}$ & $\begin{array}{l}\text { Private } \\
\text { Company }\end{array}$ & $\begin{array}{l}\text { Paul-Anders } \\
\text { Simma }\end{array}$ & 1994 & $\begin{array}{l}28 \mathrm{~min} \\
53 \mathrm{sec}\end{array}$ & $\begin{array}{l}\text { Docume } \\
\text { ntary }\end{array}$ \\
\hline
\end{tabular}




\begin{tabular}{|c|c|c|c|c|c|c|c|}
\hline & & $\begin{array}{l}\text { Saamifilm } \\
\text { Oy, Motlys } \\
\text { Film og-TV } \\
\text { produksjon, } \\
\text { A/S }\end{array}$ & & & & & \\
\hline $\begin{array}{l}\text { Ella the } \\
\text { Fishergirl }\end{array}$ & NO & $\begin{array}{l}\text { Polarfilm ja } \\
\text { TV }\end{array}$ & Unknown & Stine Sand Eira & 2007 & $29 \mathrm{~min}$ & $\begin{array}{l}\text { Docume } \\
\text { ntary }\end{array}$ \\
\hline Faces & $\mathrm{SE}$ & $\begin{array}{l}\text { Liselotte } \\
\text { Wajstedt }\end{array}$ & Individual & Liselotte Wajstedt & 2008 & $\begin{array}{l}3 \min 26 \\
\mathrm{sec}\end{array}$ & Short \\
\hline $\begin{array}{l}\text { Fear of the } \\
\text { Boundless }\end{array}$ & $\mathrm{NO}$ & $\begin{array}{l}\text { NRK/Sámi } \\
\text { Radio }\end{array}$ & State Media & Johs. Kalvemo & 2002 & $28 \mathrm{~min}$ & $\begin{array}{l}\text { Docume } \\
\text { ntary }\end{array}$ \\
\hline $\begin{array}{l}\text { Finland } \\
\text { Entered } \\
\text { Sápmi }\end{array}$ & FI & $\begin{array}{l}\text { Ima Filbma- } \\
\text { ja sátneduodji }\end{array}$ & $\begin{array}{l}\text { Private } \\
\text { Company }\end{array}$ & Anja Ahola & 2010 & 54 min & $\begin{array}{l}\text { Docume } \\
\text { ntary }\end{array}$ \\
\hline $\begin{array}{l}\text { FinnishSá } \\
\text { miFinnish }\end{array}$ & FI & $\begin{array}{l}\text { Ima Filbma- } \\
\text { ja } \\
\text { sátneduodji, } \\
\text { Inarin } \\
\text { Elokuvateolli } \\
\text { suus }\end{array}$ & $\begin{array}{l}\text { Mixed } \\
\text { (private } \\
\text { company } \\
\text { and non- } \\
\text { profit) }\end{array}$ & $\begin{array}{l}\text { Ima Aikio- } \\
\text { Arianaick }\end{array}$ & 2013 & $28 \mathrm{~min}$ & $\begin{array}{l}\text { Docume } \\
\text { ntary }\end{array}$ \\
\hline Fishing & FI & Sogsakk & Educational & Henri Portti & 2011 & $\begin{array}{l}1 \min 20 \\
\mathrm{sec}\end{array}$ & Short \\
\hline Fishing & FI & Saamelaisalu & Educational & Maria Kosonen & 2013 & $13 \mathrm{~min}$ & Short \\
\hline
\end{tabular}




\begin{tabular}{|c|c|c|c|c|c|c|c|}
\hline & & $\begin{array}{l}\text { een } \\
\text { koulutuskesk } \\
\text { us Sogsakk }\end{array}$ & & & & & \\
\hline $\begin{array}{l}\text { Fishing } \\
\text { With } \\
\text { Hooks }\end{array}$ & FI & $\begin{array}{l}\text { Inari Sámi } \\
\text { Language } \\
\text { Society }\end{array}$ & Non-profit & $\begin{array}{l}\text { Anneli } \\
\text { Lappalainen }\end{array}$ & 2010 & $5 \mathrm{~min}$ & Short \\
\hline $\begin{array}{l}\text { Fishing } \\
\text { With Nets } \\
\text { in Winter }\end{array}$ & FI & $\begin{array}{l}\text { Inari Sámi } \\
\text { Language } \\
\text { Society }\end{array}$ & Non-profit & $\begin{array}{l}\text { Anneli } \\
\text { Lappalainen }\end{array}$ & 2010 & $7 \mathrm{~min}$ & Short \\
\hline $\begin{array}{l}\text { Following } \\
\text { in the } \\
\text { Footsteps } \\
\text { of a } \\
\text { Famous } \\
\text { Reindeer } \\
\text { Herder }\end{array}$ & FI & Sogsakk & Educational & $\begin{array}{l}\text { Anne Kirste } \\
\text { Aikio }\end{array}$ & 2004 & $\begin{array}{l}2 \min 40 \\
\sec \end{array}$ & Short \\
\hline $\begin{array}{l}\text { Forest } \\
\text { Gnome }\end{array}$ & FI & Sogsakk & Educational & Janne Kyrö & 2011 & $\begin{array}{l}1 \min 30 \\
\sec \end{array}$ & Short \\
\hline $\begin{array}{l}\text { Forgivene } \\
\text { ss } \\
\text { (Andagass } \\
\text { ii) }\end{array}$ & $\mathrm{NO}$ & $\begin{array}{l}\text { Filmpool } \\
\text { Nord, Film } \\
\text { Ateljen, } \\
\text { Kautokein } \\
\text { Film AS, }\end{array}$ & $\begin{array}{l}\text { Private } \\
\text { Companies }\end{array}$ & $\begin{array}{l}\text { Nils Gaup, Kine } \\
\text { Boman }\end{array}$ & 2008 & $4 \min$ & Short \\
\hline
\end{tabular}




\begin{tabular}{|c|c|c|c|c|c|c|c|}
\hline & & $\begin{array}{l}\text { Hans-Olov } \\
\text { Utsi AB }\end{array}$ & & & & & \\
\hline Fox & FI & Sogsakk & Educational & Sunna Kitti & 2010 & $1 \mathrm{~min}$ & Short \\
\hline $\begin{array}{l}\text { Från } \\
\text { landet till } \\
\text { Country }\end{array}$ & SE & $\begin{array}{l}\text { Filmpool } \\
\text { Nord, SVT, } \\
\text { Hans-Olof } \\
\text { Utsi AB }\end{array}$ & $\begin{array}{l}\text { Mixed } \\
\text { (private } \\
\text { company, } \\
\text { state media) }\end{array}$ & Hans-Olov Utsi & 2006 & $30 \mathrm{~min}$ & Short \\
\hline $\begin{array}{l}\text { From an } \\
\text { Eye to an } \\
\text { Eye }\end{array}$ & SE & $\begin{array}{l}\text { Sámi } \\
\text { allaskuvla }\end{array}$ & Educational & $\begin{array}{l}\text { Per-Josef } \\
\text { Idivuoma }\end{array}$ & 2007 & $32 \mathrm{~min}$ & Short \\
\hline $\begin{array}{l}\text { From } \\
\text { Home to } \\
\text { Home }\end{array}$ & $\mathrm{NO}$ & $\begin{array}{l}\text { The Nordic } \\
\text { Sámi Institute }\end{array}$ & Educational & $\begin{array}{l}\text { Beate Mortensen } \\
\text { Nesheim }\end{array}$ & 2007 & $18 \mathrm{~min}$ & Short \\
\hline $\begin{array}{l}\text { From } \\
\text { Reindeer } \\
\text { Skin Into a } \\
\text { Boot }\end{array}$ & FI & Sogsakk & Educational & $\begin{array}{l}\text { Ima Aikio- } \\
\text { Arianaick }\end{array}$ & 2010 & $\begin{array}{l}13 \mathrm{~min} \\
10 \mathrm{sec}\end{array}$ & $\begin{array}{l}\text { Docume } \\
\text { ntary }\end{array}$ \\
\hline $\begin{array}{l}\text { From } \\
\text { Sápmi to } \\
\text { Alaska }\end{array}$ & $\mathrm{NO}$ & $\begin{array}{l}\text { NRK/Sámi } \\
\text { Radio }\end{array}$ & State Media & Johs. Kalvemo & 1993 & $45 \mathrm{~min}$ & $\begin{array}{l}\text { Docume } \\
\text { ntary }\end{array}$ \\
\hline $\begin{array}{l}\text { Ganda ja } \\
\text { beana }\end{array}$ & FI & $\begin{array}{l}\text { Saamelaisalu } \\
\text { een } \\
\text { koulutuskesk }\end{array}$ & Educational & Tero Titoff & 2004 & & $\begin{array}{l}\text { Children' } \\
\text { s Film }\end{array}$ \\
\hline
\end{tabular}




\begin{tabular}{|l|l|l|l|l|l|l|l|}
\hline Garanas ja & FI & Sogsakk & Educational & Tamara Navgo & 2011 & 4 min 30 & Short \\
skuolfi & & & & & sec & \\
Netting a & NO & NRK & State Media & Ronald Pulk & 2008 & 29 min & TV \\
Driver's \\
License
\end{tabular}




\begin{tabular}{|c|c|c|c|c|c|c|c|}
\hline & & & ) & & & & \\
\hline $\begin{array}{l}\text { Great } \\
\text { Grandmot } \\
\text { hers }\end{array}$ & $\mathrm{NO}$ & $\begin{array}{l}\text { Sámi } \\
\text { Kompania } \\
\text { AB }\end{array}$ & $\begin{array}{l}\text { Private } \\
\text { Company }\end{array}$ & John Erling Utsi & 2012 & $58 \mathrm{~min}$ & Feature \\
\hline $\begin{array}{l}\text { Guoros } \\
\text { Sorbmi }\end{array}$ & FI & $\begin{array}{l}\text { Tuupa } \\
\text { Records }\end{array}$ & $\begin{array}{l}\text { Music } \\
\text { Label }\end{array}$ & $\begin{array}{l}\text { Jussi Isokoski, } \\
\text { Aape Lehtinen }\end{array}$ & 2010 & $4 \mathrm{~min}$ & Short \\
\hline $\begin{array}{l}\text { Hand in } \\
\text { Hand }\end{array}$ & FI/NO & $\begin{array}{l}\text { Sámi } \\
\text { allaskuvla }\end{array}$ & Educational & Aura Nordberg & 2008 & $8 \min$ & Short \\
\hline Hare & FI & $\begin{array}{l}\text { Yleisradio/T } \\
\text { V 2, Yle } \\
\text { Sámi Radio, } \\
\text { Sogsakk }\end{array}$ & State Media & Daniel Gelencser & 2008 & $1 \mathrm{~min}$ & Short \\
\hline $\begin{array}{l}\text { Heads } \\
\text { Down }\end{array}$ & FI & & Unknown & $\begin{array}{l}\text { Ima Aikio- } \\
\text { Arianaick }\end{array}$ & 2013 & $2 \min$ & Short \\
\hline $\begin{array}{l}\text { Hello } \\
\text { There } \\
\text { Jambo! } \\
\text { How's My } \\
\text { Reindeer } \\
\text { Doing? }\end{array}$ & FI & $\begin{array}{l}\text { Saamelaisalu } \\
\text { een } \\
\text { koulutuskesk } \\
\text { us SAKK }\end{array}$ & Educational & $\begin{array}{l}\text { Ann-Christine } \\
\text { Haupt }\end{array}$ & 2004 & $8 \mathrm{~min}$ & Short \\
\hline $\begin{array}{l}\text { Helping } \\
\text { the World }\end{array}$ & $\mathrm{NO}$ & $\begin{array}{l}\text { Filmpool } \\
\text { Nord, NRK, } \\
\text { Filbmagoahti }\end{array}$ & $\begin{array}{l}\text { Mixed } \\
\text { (private } \\
\text { companies, }\end{array}$ & $\begin{array}{l}\text { John Erling Utsi, } \\
\text { Liv-Inger Somby }\end{array}$ & 2004 & $30 \mathrm{~min}$ & $\begin{array}{l}\text { Docume } \\
\text { ntary }\end{array}$ \\
\hline
\end{tabular}




\begin{tabular}{|c|c|c|c|c|c|c|c|}
\hline & & $\begin{array}{l}\text { AS, Zentropa } \\
\text { Entertainmen } \\
\text { ts } 7 \mathrm{ApS}, \\
\text { Nordland } \\
\text { College of } \\
\text { Art and Film, } \\
\text { Film Form } \\
\text { AB }\end{array}$ & state media) & & & & \\
\hline $\begin{array}{l}\text { Herdswom } \\
\text { an }\end{array}$ & SE & $\begin{array}{l}\text { Filmpool } \\
\text { Nord, Film } \\
\text { Ateljen }\end{array}$ & $\begin{array}{l}\text { Private } \\
\text { company }\end{array}$ & Kine Boman & 2008 & $58 \mathrm{~min}$ & Short \\
\hline Heritage & FI & Sogsakk & Educational & Kirsti Länsman & 2007 & $5 \min$ & Short \\
\hline $\begin{array}{l}\text { Hi there, } \\
\text { little kid }\end{array}$ & $\mathrm{NO}$ & & Unknown & Ken Are Bongo & 2005 & $5 \min$ & Short \\
\hline $\begin{array}{l}\text { Historical } \\
\text { Journey } \\
\text { By Horse }\end{array}$ & $\mathrm{NO}$ & $\begin{array}{l}\text { Filmpool } \\
\text { Nord, } \\
\text { NRK/Sámi } \\
\text { Radio, NRK, } \\
\text { Filbmagoahti } \\
\text { AS, Zentropa } \\
\text { Entertainmen } \\
\text { ts7 ApS, } \\
\text { Sogsakk, }\end{array}$ & $\begin{array}{l}\text { Mixed } \\
\text { (Private } \\
\text { company, } \\
\text { state media, } \\
\text { educational) }\end{array}$ & Johs. Kalvemo & 2003 & $30 \mathrm{~min}$ & $\begin{array}{l}\text { TV } \\
\text { Producti } \\
\text { on }\end{array}$ \\
\hline
\end{tabular}




\begin{tabular}{|c|c|c|c|c|c|c|c|}
\hline & & $\begin{array}{l}\text { Nordland } \\
\text { College of } \\
\text { Art and Film, } \\
\text { Film Form } \\
\text { AB }\end{array}$ & & & & & \\
\hline $\begin{array}{l}\text { Hodet } \\
\text { Over } \\
\text { Vannet }\end{array}$ & $\mathrm{NO}$ & $\begin{array}{l}\text { Filmkamerate } \\
\text { ne AS, } \\
\text { Svensk } \\
\text { Filmindustri }\end{array}$ & $\begin{array}{l}\text { Private } \\
\text { company }\end{array}$ & Nils Gaup & 1993 & $\begin{array}{l}1 \mathrm{~h} 40 \\
\min \end{array}$ & $\begin{array}{l}\text { Docume } \\
\text { ntary }\end{array}$ \\
\hline $\begin{array}{l}\text { House of } \\
\text { Winds }\end{array}$ & $\mathrm{NO}$ & JabFilm & $\begin{array}{l}\text { Private } \\
\text { company }\end{array}$ & $\begin{array}{l}\text { Magnar } \\
\text { Mikkelsen }\end{array}$ & 2009 & $58 \mathrm{~min}$ & $\begin{array}{l}\text { Docume } \\
\text { ntary }\end{array}$ \\
\hline $\begin{array}{l}\text { How the } \\
\text { Lavvo is } \\
\text { also a } \\
\text { Goahti }\end{array}$ & $\mathrm{NO}$ & $\begin{array}{l}\text { Sámi } \\
\text { Allaskuvla, } \\
\text { ISF AS, } \\
\text { Traditional } \\
\text { Knowledge } \\
\text { Project }\end{array}$ & $\begin{array}{l}\text { Mixed } \\
\text { (private } \\
\text { company, } \\
\text { educational, } \\
\text { non- } \\
\text { government } \\
\text { organization } \\
\text {, unknown) }\end{array}$ & $\begin{array}{l}\text { Anne Merete A. } \\
\text { Gaup }\end{array}$ & 2009 & $11 \mathrm{~min}$ & Short \\
\hline $\begin{array}{l}\text { How to } \\
\text { Dance } \\
\text { With } \\
\text { Bears }\end{array}$ & FI & $\begin{array}{l}\text { Saami-Filmi } \\
\text { Oy }\end{array}$ & $\begin{array}{l}\text { Private } \\
\text { company }\end{array}$ & $\begin{array}{l}\text { Paul-Anders } \\
\text { Simma }\end{array}$ & 2008 & $48 \mathrm{~min}$ & $\begin{array}{l}\text { Docume } \\
\text { ntary }\end{array}$ \\
\hline
\end{tabular}




\begin{tabular}{|c|c|c|c|c|c|c|c|}
\hline $\begin{array}{l}\text { Feel } \\
\text { Nature } \\
\text { Breathing }\end{array}$ & NO & $\begin{array}{l}\text { NRK/Sámi } \\
\text { Radio }\end{array}$ & State Media & Johs. Kalvemo & 2002 & $28 \mathrm{~min}$ & $\begin{array}{l}\text { Docume } \\
\text { ntary }\end{array}$ \\
\hline $\begin{array}{l}\text { I Like the } \\
\text { Green } \\
\text { Colour }\end{array}$ & $\begin{array}{l}\mathrm{NO} / \mathrm{S} \\
\text { ápmi }\end{array}$ & $\begin{array}{l}\text { Ninne Film } \\
\text { AS }\end{array}$ & $\begin{array}{l}\text { Private } \\
\text { Company }\end{array}$ & $\begin{array}{l}\text { Nils John } \\
\text { Porsanger, Eilif } \\
\text { Aslaksen }\end{array}$ & 2004 & $30 \mathrm{~min}$ & $\begin{array}{l}\text { Docume } \\
\text { ntary }\end{array}$ \\
\hline $\begin{array}{l}\text { I Will } \\
\text { Miss You }\end{array}$ & FI & $\begin{array}{l}\text { Saamelaisalu } \\
\text { een } \\
\text { koulutuskesk } \\
\text { us Sogsakk }\end{array}$ & & Sunna Kitti & 2012 & $4 \min$ & Short \\
\hline $\begin{array}{l}\text { Inari Sámi } \\
\text { - } \\
\text { Endangere } \\
\text { d But Not } \\
\text { Dying }\end{array}$ & FI & $\begin{array}{l}\text { Saamelaisalu } \\
\text { een } \\
\text { koulutuskesk } \\
\text { us SAKK }\end{array}$ & Educational & $\begin{array}{l}\text { Ima Aikio- } \\
\text { Arianaick }\end{array}$ & 2004 & $7 \mathrm{~min}$ & Short \\
\hline $\begin{array}{l}\text { Internet } \\
\text { Addict }\end{array}$ & FI & Sogsakk & Educational & Matti Kukkola & 2010 & $1 \mathrm{~min}$ & Short \\
\hline $\begin{array}{l}\text { Joiking } \\
\text { Lawyer }\end{array}$ & $\mathrm{NO}$ & NRK & State media & Stein-Åge Isaksen & 2002 & $29 \mathrm{~min}$ & $\begin{array}{l}\text { Docume } \\
\text { ntary }\end{array}$ \\
\hline $\begin{array}{l}\text { Jokers on } \\
\text { the Road }\end{array}$ & $\mathrm{NO}$ & NRK & State media & $\begin{array}{l}\text { Nils John } \\
\text { Porsanger }\end{array}$ & 2003 & $30 \mathrm{~min}$ & $\begin{array}{l}\text { TV } \\
\text { Producti } \\
\text { on }\end{array}$ \\
\hline Jokers on & $\mathrm{NO}$ & NRK & State media & Nils John & 2003 & $39 \mathrm{~min}$ & Short \\
\hline
\end{tabular}




\begin{tabular}{|c|c|c|c|c|c|c|c|}
\hline the Road & & & & Porsanger & & & \\
\hline $\begin{array}{l}\text { Jon vis-a- } \\
\text { vis the } \\
\text { winds }\end{array}$ & $\begin{array}{l}\mathrm{SE} / \mathrm{F} \\
\mathrm{R}\end{array}$ & $\begin{array}{l}\text { Sámi } \\
\text { Kompania } \\
\text { AB, } \\
\text { Arwestud } \\
\text { Films }\end{array}$ & $\begin{array}{l}\text { Private } \\
\text { companies }\end{array}$ & Corto Fajal & 2010 & $59 \mathrm{~min}$ & $\begin{array}{l}\text { Docume } \\
\text { ntary }\end{array}$ \\
\hline $\begin{array}{l}\text { Karine } \\
\text { and the } \\
\text { Lost } \\
\text { Rhyme }\end{array}$ & $\mathrm{NO}$ & Siivet AS & $\begin{array}{l}\text { Private } \\
\text { company }\end{array}$ & $\begin{array}{l}\text { Anstein } \\
\text { Mikkelsen }\end{array}$ & 2007 & $22 \mathrm{~min}$ & Short \\
\hline $\begin{array}{l}\text { Kiruna } \\
\text { Space } \\
\text { Road }\end{array}$ & SE & $\begin{array}{l}\text { LittleBig } \\
\text { Productions }\end{array}$ & $\begin{array}{l}\text { Private } \\
\text { company }\end{array}$ & Liselotte Wajstedt & 2013 & $52 \mathrm{~min}$ & Feature \\
\hline Kiurrels & FI & $\begin{array}{l}\text { Saamelaisalu } \\
\text { een } \\
\text { koulutuskesk } \\
\text { us SAKK, } \\
\text { Samediggi }\end{array}$ & $\begin{array}{l}\text { Mixed } \\
\text { (educational } \\
\text {, unknown) }\end{array}$ & Pauliina Fedoroff & 2007 & $3 \min$ & Short \\
\hline $\begin{array}{l}\text { Knute Are } \\
\text { Explores } \\
\text { Sámi } \\
\text { Words }\end{array}$ & $\mathrm{NO}$ & Nuoraj TV & & Maria Mikkelsen & 2010 & $2 \min$ & Short \\
\hline Koltloop & SE & Liselotte & Individual & Liselotte Wajstedt & 2005 & $3 \min$ & Docume \\
\hline
\end{tabular}




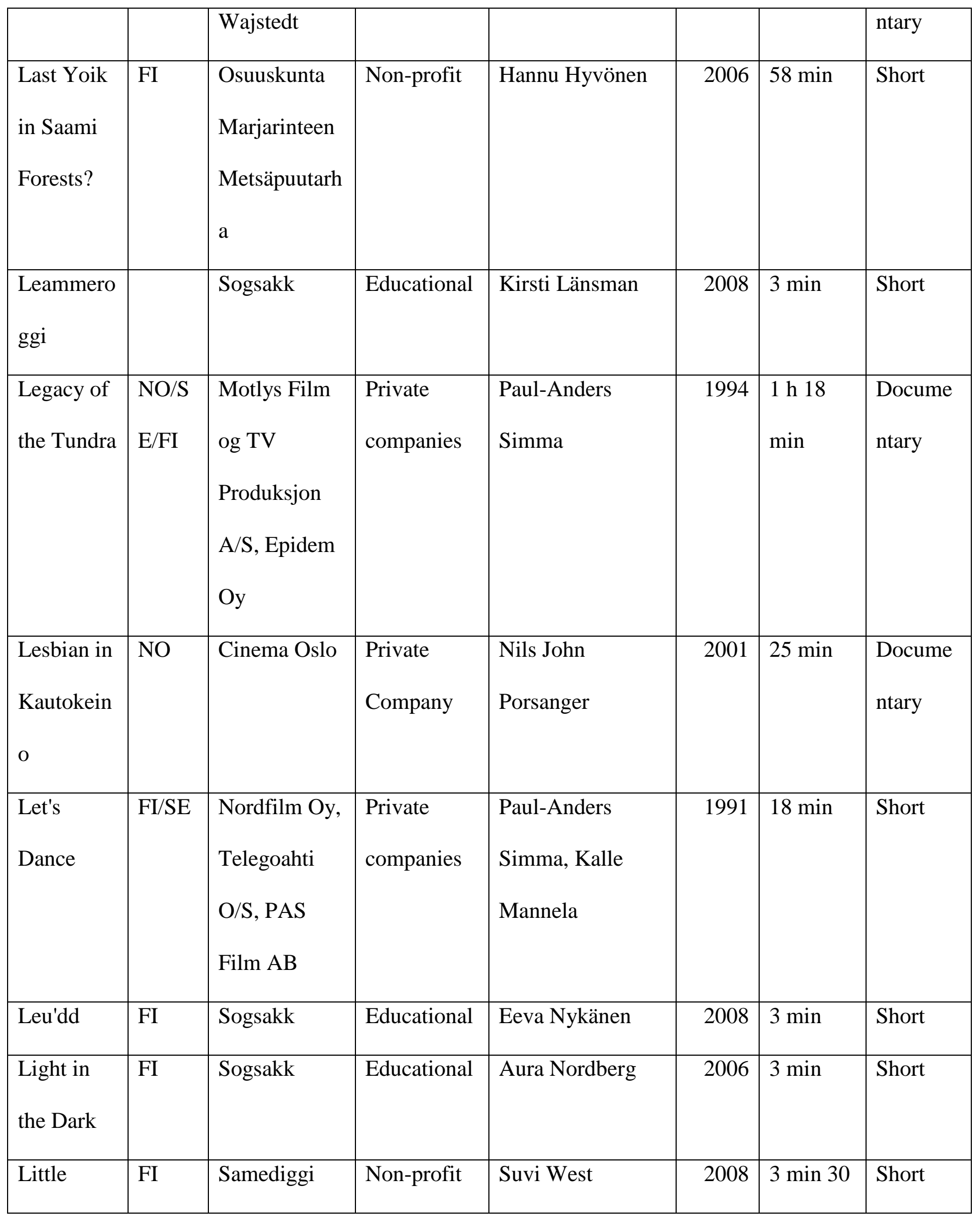




\begin{tabular}{|c|c|c|c|c|c|c|c|}
\hline Sister & & & & & & $\mathrm{sec}$ & \\
\hline $\begin{array}{l}\text { Longing } \\
\text { for Silence }\end{array}$ & NO & & Unknown & $\begin{array}{l}\text { Niels Ovlla } \\
\text { Dunfjell }\end{array}$ & 2011 & $4 \min$ & Short \\
\hline $\begin{array}{l}\text { Looking } \\
\text { Forward }\end{array}$ & FI & Sogsakk & Educational & $\begin{array}{l}\text { Sinituuli } \\
\text { Kalttopää, Emma } \\
\text { Eira, Mira Kärki }\end{array}$ & 2010 & $1 \mathrm{~min}$ & Short \\
\hline $\begin{array}{l}\text { Love is } \\
\text { Lost }\end{array}$ & FI & $\begin{array}{l}\text { Saamelaisalu } \\
\text { een } \\
\text { koulutuskesk } \\
\text { us Sogsakk }\end{array}$ & Educational & Marjo Ulkuniemi & 2013 & $12 \mathrm{~min}$ & Short \\
\hline $\begin{array}{l}\text { Made in } \\
\text { Sápmi }\end{array}$ & FI & $\begin{array}{l}\text { Saamelaisalu } \\
\text { een } \\
\text { koulutuskesk } \\
\text { us Sogsakk }\end{array}$ & Educational & Jarkko Remahl & 2013 & $21 \mathrm{~min}$ & Short \\
\hline $\begin{array}{l}\text { Making } \\
\text { Delicacies }\end{array}$ & FI & $\begin{array}{l}\text { Inari Sámi } \\
\text { Language } \\
\text { Society }\end{array}$ & Non-profit & $\begin{array}{l}\text { Anneli } \\
\text { Lappalainen }\end{array}$ & 2010 & $13 \mathrm{~min}$ & Short \\
\hline $\begin{array}{l}\text { Man was } \\
\text { Born in } \\
\text { the Sea }\end{array}$ & FI & Sogsakk & Educational & Sunna Kitti & 2008 & $1 \mathrm{~min}$ & Short \\
\hline $\begin{array}{l}\text { Meahcce } \\
\text { vulle }\end{array}$ & & SVT Sápmi & State Media & & 2002 & $30 \mathrm{~min}$ & $\begin{array}{l}\text { TV } \\
\text { Producti } \\
\text { on }\end{array}$ \\
\hline
\end{tabular}




\begin{tabular}{|c|c|c|c|c|c|c|c|}
\hline Meeting & FI & Sogsakk & Educational & Marjo Ulkuniemi & 2011 & $2 \min$ & Short \\
\hline $\begin{array}{l}\text { Min } \\
\text { guovlu }\end{array}$ & $\mathrm{NO}$ & Egil Pedersen & Individual & Egil Pedersen & 2013 & $4 \min$ & Short \\
\hline $\begin{array}{l}\text { Miniature } \\
\text { Variety } \\
\text { Show }\end{array}$ & FI & $\begin{array}{l}\text { Saamelaisalu } \\
\text { een } \\
\text { koulutuskesk } \\
\text { us Sogsakk }\end{array}$ & Educational & Susanna Rauno & 2012 & $18 \mathrm{~min}$ & Short \\
\hline Mirror & FI & Sogsakk & Educational & Mikkal Morottaja & 2007 & $4 \min$ & Short \\
\hline $\begin{array}{l}\text { Misery } \\
\text { Harbour }\end{array}$ & NO & Motlys A/S & $\begin{array}{l}\text { Private } \\
\text { company }\end{array}$ & Nils Gaup & 1999 & $\begin{array}{l}1 \mathrm{~h} 40 \\
\min \end{array}$ & $\begin{array}{l}\text { Docume } \\
\text { ntary }\end{array}$ \\
\hline $\begin{array}{l}\text { Mon, } \\
\text { Mollett ja } \\
\text { Don }\end{array}$ & SE & IdiStudios & Educational & $\begin{array}{l}\text { Per-Josef } \\
\text { Idivuoma }\end{array}$ & 2011 & $5 \mathrm{~min}$ & Short \\
\hline $\begin{array}{l}\text { Mosquito } \\
\text { Shelter }\end{array}$ & $\begin{array}{l}\text { FI/NO } \\
/ \mathrm{SE}\end{array}$ & $\begin{array}{l}\text { SVT, YLE, } \\
\text { NRK }\end{array}$ & State media & $\begin{array}{l}\text { lisko Sara, Kalle } \\
\text { Mannela, Frode } \\
\text { Grönmo }\end{array}$ & 1987 & $25 \mathrm{~min}$ & $\begin{array}{l}\text { Docume } \\
\text { ntary }\end{array}$ \\
\hline $\begin{array}{l}\text { Mother's } \\
\text { Words }\end{array}$ & NO & Siivet AS & $\begin{array}{l}\text { Private } \\
\text { company }\end{array}$ & $\begin{array}{l}\text { Anstein } \\
\text { Mikkelsen }\end{array}$ & 2009 & $28 \mathrm{~min}$ & $\begin{array}{l}\text { Docume } \\
\text { ntary }\end{array}$ \\
\hline Mountain & $\mathrm{SE}$ & Tor Lundberg & Individual & Tor Lundberg & 2012 & $4 \min$ & Short \\
\hline $\begin{array}{l}\text { Mrs and } \\
\text { Mrs Sarri }\end{array}$ & SE & $\begin{array}{l}\text { Sámi } \\
\text { Kompania } \\
\text { AB }\end{array}$ & $\begin{array}{l}\text { Private } \\
\text { company }\end{array}$ & Lisbeth Blind & 2006 & $28 \mathrm{~min}$ & Short \\
\hline Muhto & FI & Saamelaisalu & Educational & Suvi West & 2004 & $7 \mathrm{~min}$ & Short \\
\hline
\end{tabular}




\begin{tabular}{|c|c|c|c|c|c|c|c|}
\hline $\begin{array}{l}\text { stuoramus } \\
\text { dain lea } \\
\text { rahkesvuot } \\
\text { a }\end{array}$ & & $\begin{array}{l}\text { een } \\
\text { koulutuskesk } \\
\text { us SAKK }\end{array}$ & & & & & \\
\hline $\begin{array}{l}\text { Mummon } \\
\text { kuumat } \\
\text { jalat }\end{array}$ & FI & Sogsakk & Educational & Sunna Kitti & 2011 & $4 \mathrm{~min}$ & Short \\
\hline My Anar & FI & Sogsakk & Educational & Irene Länsman & 2010 & $2 \mathrm{~min}$ & Short \\
\hline $\begin{array}{l}\text { My Car } \\
\text { and the } \\
\text { Song of a } \\
\text { Girl }\end{array}$ & $\mathrm{NO}$ & & Unknown & Ken Are Bongo & 2009 & $3 \mathrm{~min}$ & Short \\
\hline $\begin{array}{l}\text { My Deer } \\
\text { Ones }\end{array}$ & $\mathrm{NO}$ & $\begin{array}{l}\text { Movttegis } \\
\text { Máhtte } \\
\text { Media }\end{array}$ & Individual & $\begin{array}{l}\text { Mattias Sikku } \\
\text { Valio }\end{array}$ & 2012 & $40 \mathrm{~min}$ & Feature \\
\hline $\begin{array}{l}\text { My } \\
\text { Family } \\
\text { Portrait }\end{array}$ & $\mathrm{NO}$ & Pandora Film & $\begin{array}{l}\text { Private } \\
\text { company }\end{array}$ & $\begin{array}{l}\text { Yvonne } \\
\text { Thommassen }\end{array}$ & 2013 & $59 \mathrm{~min}$ & Feature \\
\hline $\begin{array}{l}\text { My } \\
\text { Grandmot } \\
\text { her and I }\end{array}$ & SE & $\begin{array}{l}\text { Luleå } \\
\text { University of } \\
\text { Technology, } \\
\text { Department } \\
\text { of Med }\end{array}$ & Educational & $\begin{array}{l}\text { Ann-Christine } \\
\text { Haupt }\end{array}$ & 2006 & $13 \mathrm{~min}$ & $\begin{array}{l}\text { Docume } \\
\text { ntary }\end{array}$ \\
\hline
\end{tabular}




\begin{tabular}{|c|c|c|c|c|c|c|c|}
\hline $\begin{array}{l}\text { My Hearts } \\
\text { Voice }\end{array}$ & $\mathrm{SE}$ & $\begin{array}{l}\text { Marit } \\
\text { Kuhmunen }\end{array}$ & Individual & Marit Kuhmunen & 2009 & $14 \mathrm{~min}$ & Short \\
\hline My Hobby & FI & Sogsakk & Educational & Eero Mattus & 2008 & $\begin{array}{l}1 \min 30 \\
\mathrm{sec}\end{array}$ & Short \\
\hline $\begin{array}{l}\text { My Home } \\
\text { is In My } \\
\text { Heart }\end{array}$ & $\mathrm{NO} / \mathrm{FI}$ & $\begin{array}{l}\text { NRK/Sámi } \\
\text { Radio }\end{array}$ & State media & Liv-Inger Somby & 2001 & $58 \mathrm{~min}$ & $\begin{array}{l}\text { Docume } \\
\text { ntary }\end{array}$ \\
\hline $\begin{array}{l}\text { Neeta } \\
\text { Inari }\end{array}$ & $\mathrm{SE}$ & IdiStudios & $\begin{array}{l}\text { Private } \\
\text { company }\end{array}$ & $\begin{array}{l}\text { Per-Josef } \\
\text { Idivuoma }\end{array}$ & 2013 & $6 \min$ & Short \\
\hline Niiles & FI & Sogsakk & Educational & Jouni West & 2008 & $1 \mathrm{~min}$ & Short \\
\hline $\begin{array}{l}\text { Nils } \\
\text { Nilsson } \\
\text { Skum }\end{array}$ & SE & $\begin{array}{l}\text { Sámi } \\
\text { Kompania } \\
\text { AB }\end{array}$ & $\begin{array}{l}\text { Private } \\
\text { company }\end{array}$ & $\begin{array}{l}\text { John Erling Utsi, } \\
\text { Lisbeth Blind }\end{array}$ & 2002 & $30 \mathrm{~min}$ & $\begin{array}{l}\text { Docume } \\
\text { ntary }\end{array}$ \\
\hline Non Profit & FI & $\begin{array}{l}\text { Periferia } \\
\text { Productions } \\
\text { Oy }\end{array}$ & $\begin{array}{l}\text { Private } \\
\text { company }\end{array}$ & Pauliina Fedoroff & 2008 & $\begin{array}{l}1 \mathrm{hr} 26 \\
\min \end{array}$ & $\begin{array}{l}\text { Docume } \\
\text { ntary }\end{array}$ \\
\hline $\begin{array}{l}\text { Non-Sámi } \\
\text { Women }\end{array}$ & FI & Sogsakk & Educational & Kauko Mustonen & 2009 & $10 \mathrm{~min}$ & Short \\
\hline Ofelas & FI & $\begin{array}{l}\text { Saamelaisalu } \\
\text { een } \\
\text { koulutuskesk } \\
\text { us SAKK }\end{array}$ & Educational & $\begin{array}{l}\text { Ritva Torikka- } \\
\text { Gelencser, Petra } \\
\text { Magga }\end{array}$ & 2008 & $3 \min$ & $\begin{array}{l}\text { Children' } \\
\text { s Film }\end{array}$ \\
\hline Olbmo & FI & Yleisradio/T & State media & Esko Faven & 1996 & $50 \mathrm{~min}$ & Docume \\
\hline
\end{tabular}




\begin{tabular}{|c|c|c|c|c|c|c|c|}
\hline Dolggit & & $\mathrm{V} 2$ & & & & & ntary \\
\hline $\begin{array}{l}\text { On the } \\
\text { Wave of } \\
\text { Life }\end{array}$ & FI & Sogsakk & Educational & Kirsti Länsman & 2009 & $8 \mathrm{~min}$ & Short \\
\hline $\begin{array}{l}\text { One Shot } \\
\text { Closer }\end{array}$ & SE & $\begin{array}{l}\text { Kalix } \\
\text { Folkhögskola }\end{array}$ & Educational & $\begin{array}{l}\text { Lars Svonni, Elle } \\
\text { Porsanger }\end{array}$ & 2008 & $15 \mathrm{~min}$ & $\begin{array}{l}\text { Docume } \\
\text { ntary }\end{array}$ \\
\hline $\begin{array}{l}\text { Passing } \\
\text { On the } \\
\text { Language }\end{array}$ & FI & $\begin{array}{l}\text { Anarâškielâ } \\
\text { servi ry }\end{array}$ & Non-profit & $\begin{array}{l}\text { Ima Aikio- } \\
\text { Arianaick, Anneli } \\
\text { Lappalainen }\end{array}$ & 2013 & $25 \mathrm{~min}$ & $\begin{array}{l}\text { Docume } \\
\text { ntary }\end{array}$ \\
\hline $\begin{array}{l}\text { Path of } \\
\text { Life }\end{array}$ & FI & $\begin{array}{l}\text { Saamelaisalu } \\
\text { een } \\
\text { koulutuskesk } \\
\text { us SAKK }\end{array}$ & Educational & $\begin{array}{l}\text { Erkki Feodoroff, } \\
\text { Aki Harju }\end{array}$ & 2012 & & Short \\
\hline Pathfinder & $\mathrm{NO}$ & $\begin{array}{l}\text { Filmkamerate } \\
\text { ne AS }\end{array}$ & $\begin{array}{l}\text { Private } \\
\text { company }\end{array}$ & Nils Gaup & 1987 & $\begin{array}{l}1 \mathrm{~h} 28 \\
\min \end{array}$ & Feature \\
\hline Pauli & FI & $\begin{array}{l}\text { Saamelaisalu } \\
\text { een } \\
\text { koulutuskesk } \\
\text { us Sogsakk }\end{array}$ & Educational & Henri Portti & 2012 & $12 \mathrm{~min}$ & $\begin{array}{l}\text { Docume } \\
\text { ntary }\end{array}$ \\
\hline Pei'vv Past & FI & $\begin{array}{l}\text { Tampereen } \\
\text { ammattikorke } \\
\text { akoulu } \\
\text { TAMK/Taide }\end{array}$ & Educational & Katja Gauriloff & 2004 & $14 \mathrm{~min}$ & Short \\
\hline
\end{tabular}




\begin{tabular}{|c|c|c|c|c|c|c|c|}
\hline & & ja viestin & & & & & \\
\hline Pesku & FI & Sogsakk & Educational & Daniel Gelencser & 2008 & $5 \mathrm{~min}$ & Short \\
\hline $\begin{array}{l}\text { Peter Gets } \\
\text { Even }\end{array}$ & $\mathrm{NO}$ & ISF AS & $\begin{array}{l}\text { Non- } \\
\text { government } \\
\text { organization } \\
\text { (mixed } \\
\text { funding) }\end{array}$ & $\begin{array}{l}\text { Sven Thomas } \\
\text { Holm, Anja Marja } \\
\text { Keskitalo, Johan } \\
\text { Eivind Bals, Ole } \\
\text { Marius Holm, } \\
\text { Inga Birgitte } \\
\text { Haetta, Maret } \\
\text { Michelle, Mienna } \\
\text { Eira, Annele } \\
\text { Gaup }\end{array}$ & 2009 & $7 \mathrm{~min}$ & Short \\
\hline $\begin{array}{l}\text { Pictures of } \\
\text { My Life }\end{array}$ & SE & SVT Sápmi & State media & $\begin{array}{l}\text { Bjarne Store- } \\
\text { Jakobsen }\end{array}$ & 2002 & $30 \mathrm{~min}$ & $\begin{array}{l}\text { Docume } \\
\text { ntary }\end{array}$ \\
\hline $\begin{array}{l}\text { Pilvi } \\
\text { Aloittaa } \\
\text { yliopistoss } \\
\text { a }\end{array}$ & FI & Samediggi & Educational & Suvi West & 2008 & $\begin{array}{l}1 \min 40 \\
\sec \end{array}$ & Short \\
\hline $\begin{array}{l}\text { Playing } \\
\text { With Fire }\end{array}$ & $\mathrm{NO}$ & $\begin{array}{l}\text { Nordland } \\
\text { College of } \\
\text { Art and Film }\end{array}$ & Educational & $\begin{array}{l}\text { Anne Merete } \\
\text { Gaup }\end{array}$ & 2012 & $16 \mathrm{~min}$ & Short \\
\hline $\begin{array}{l}\text { Poazovazz } \\
\text { i }\end{array}$ & FI & $\begin{array}{l}\text { Suomen } \\
\text { Televisio }\end{array}$ & Unknown & Antero Ruuhonen & 1963 & $30 \mathrm{~min}$ & $\begin{array}{l}\text { Docume } \\
\text { ntary }\end{array}$ \\
\hline
\end{tabular}




\begin{tabular}{|c|c|c|c|c|c|c|c|}
\hline $\begin{array}{l}\text { Portraits } \\
\text { from } \\
\text { Varangerfj } \\
\text { ord }\end{array}$ & $\mathrm{NO}$ & Egil Pedersen & Individual & Egil Pedersen & 2013 & $13 \mathrm{~min}$ & $\begin{array}{l}\text { Docume } \\
\text { ntary }\end{array}$ \\
\hline $\begin{array}{l}\text { Quite an } \\
\text { Ugly Frog }\end{array}$ & FI & Sogsakk & Educational & $\begin{array}{l}\text { Jouni West, Pia } \\
\text { Mikkilä }\end{array}$ & 2010 & $4 \min$ & Short \\
\hline Radja & FI/SL & $\begin{array}{l}\text { Academy Of } \\
\text { Arts }\end{array}$ & Educational & Lukas Palatinus & 2011 & $20 \mathrm{~min}$ & $\begin{array}{l}\text { Docume } \\
\text { ntary }\end{array}$ \\
\hline $\begin{array}{l}\text { Rahkistan, } \\
\text { in mun ge }\end{array}$ & $\mathrm{NO}$ & & Unknown & Geir Tore Holm & 2005 & $5 \mathrm{~min}$ & Short \\
\hline Reborn & FI & Sogsakk & Educational & Suvi Kivelä & 2010 & $10 \mathrm{~min}$ & Short \\
\hline $\begin{array}{l}\text { Reindeer } \\
\text { Princess }\end{array}$ & $\mathrm{NO}$ & $\begin{array}{l}\text { Ninne Film } \\
\text { AS }\end{array}$ & $\begin{array}{l}\text { Private } \\
\text { company }\end{array}$ & $\begin{array}{l}\text { Nils John } \\
\text { Porsanger }\end{array}$ & 2007 & $28 \mathrm{~min}$ & $\begin{array}{l}\text { Docume } \\
\text { ntary }\end{array}$ \\
\hline $\begin{array}{l}\text { Respect } \\
\text { Sámi } \\
\text { Culture! }\end{array}$ & SE & & & Nils Anders Inga & 2008 & $10 \mathrm{~min}$ & Short \\
\hline $\begin{array}{l}\text { Retaliation } \\
\text { for a } \\
\text { Greater } \\
\text { Good }\end{array}$ & SE & IdiStudios & $\begin{array}{l}\text { Private } \\
\text { company }\end{array}$ & $\begin{array}{l}\text { Per-Josef } \\
\text { Idivuoma }\end{array}$ & 2012 & $9 \min$ & Short \\
\hline Ride Free & FI & $\begin{array}{l}\text { Saamelaisalu } \\
\text { een } \\
\text { koulutuskesk }\end{array}$ & Educational & Kevin Francett & 2013 & $15 \mathrm{~min}$ & Short \\
\hline
\end{tabular}




\begin{tabular}{|c|c|c|c|c|c|c|c|}
\hline & & us Sogsakk & & & & & \\
\hline $\begin{array}{l}\text { Ritva and } \\
\text { Xia }\end{array}$ & FI & $\begin{array}{l}\text { Saamelaisalu } \\
\text { een } \\
\text { koulutuskesk } \\
\text { us SAKK }\end{array}$ & Educational & Ritva Torikka & 2002 & $15 \mathrm{~min}$ & $\begin{array}{l}\text { Docume } \\
\text { ntary }\end{array}$ \\
\hline $\begin{array}{l}\text { Rock } \\
\text { Reindeer }\end{array}$ & FI & Sogsakk & Educational & Jarno Kiimala & 2010 & $2 \min$ & Short \\
\hline Rockabilly & SE & $\begin{array}{l}\text { Liselotte } \\
\text { Wajstedt }\end{array}$ & Individual & Liselotte Wajstedt & 2006 & $9 \min$ & Short \\
\hline $\begin{array}{l}\text { Rome, } \\
\text { Here I } \\
\text { Come! }\end{array}$ & FI & Sogsakk & Educational & Sirpa Taivainen & 2007 & $6 \mathrm{~min}$ & Short \\
\hline $\begin{array}{l}\text { Sacred } \\
\text { Stone }\end{array}$ & SE & $\begin{array}{l}\text { Migma Film } \\
\mathrm{AB}\end{array}$ & $\begin{array}{l}\text { Private } \\
\text { company }\end{array}$ & Åsa Simma & 1996 & $24 \mathrm{~min}$ & Short \\
\hline Safari & FI & Sogsakk & Educational & Jarkko Remahl & 2011 & $1 \mathrm{~min}$ & Short \\
\hline $\begin{array}{l}\text { Sámi } \\
\text { Belly } \\
\text { Dancer }\end{array}$ & $\mathrm{NO}$ & NRK & State media & $\begin{array}{l}\text { Niels Ovlla } \\
\text { Dunfjell }\end{array}$ & 2013 & $4 \min$ & Short \\
\hline $\begin{array}{l}\text { Sámi } \\
\text { Daughter } \\
\text { Yoik }\end{array}$ & SE & $\begin{array}{l}\text { LittleBig } \\
\text { Productions }\end{array}$ & Individual & Liselotte Wajstedt & 2007 & $58 \mathrm{~min}$ & Short \\
\hline $\begin{array}{l}\text { Sámi } \\
\text { Drum }\end{array}$ & FI & Sogsakk & Educational & Sampo Issakainen & 2010 & $\begin{array}{l}1 \min 58 \\
\sec \end{array}$ & Short \\
\hline
\end{tabular}




\begin{tabular}{|c|c|c|c|c|c|c|c|}
\hline $\begin{array}{l}\text { Sámi } \\
\text { manat } \\
\text { helssegis }\end{array}$ & FI & $\begin{array}{l}\text { Saamelaisalu } \\
\text { een } \\
\text { koulutuskesk } \\
\text { us SAKK }\end{array}$ & Educational & Katja Gauriloff & 2006 & $15 \mathrm{~min}$ & $\begin{array}{l}\text { Docume } \\
\text { ntary }\end{array}$ \\
\hline $\begin{array}{l}\text { Sámi } \\
\text { moment }\end{array}$ & $\mathrm{NO}$ & $\begin{array}{l}\text { Kautokeino } \\
\text { Film AS }\end{array}$ & $\begin{array}{l}\text { Private } \\
\text { company }\end{array}$ & Ken Are Bongo & 2011 & $6 \mathrm{~min}$ & Short \\
\hline $\begin{array}{l}\text { Sámi } \\
\text { vision, a } \\
\text { lost vision }\end{array}$ & FI & Aslak Paltto & Individual & Aslak Paltto & 2009 & $27 \mathrm{~min}$ & $\begin{array}{l}\text { Docume } \\
\text { ntary }\end{array}$ \\
\hline $\begin{array}{l}\text { Sámi } \\
\text { Winter } \\
\text { Clothing }\end{array}$ & FI & $\begin{array}{l}\text { Sámi Siida } \\
\text { Ry }\end{array}$ & Non-profit & $\begin{array}{l}\text { Ima Aikio- } \\
\text { Arianaick }\end{array}$ & 2006 & $13 \mathrm{~min}$ & Short \\
\hline $\begin{array}{l}\text { Sammeli's } \\
\text { Law }\end{array}$ & FI & $\begin{array}{l}\text { Yleisradio } \\
\text { Oy }\end{array}$ & State media & $\begin{array}{l}\text { Juhani } \\
\text { Nousuniemi, Aila } \\
\text { Rantala }\end{array}$ & 1976 & $42 \mathrm{~min}$ & $\begin{array}{l}\text { Docume } \\
\text { ntary }\end{array}$ \\
\hline Sapmelas & FI & $\begin{array}{l}\text { Giron-filmi } \\
\text { Oy, Liisa } \\
\text { Holmberg }\end{array}$ & $\begin{array}{l}\text { Mixed } \\
\text { (private } \\
\text { company, } \\
\text { individual) }\end{array}$ & $\begin{array}{l}\text { Markku } \\
\text { Lehmuskallio, } \\
\text { Anastasia Lapsui }\end{array}$ & 2006 & $82 \mathrm{~min}$ & $\begin{array}{l}\text { Docume } \\
\text { ntary }\end{array}$ \\
\hline Sauna & FI & Sogsakk & Educational & Jarno Kiimala & 2010 & $2 \min$ & Short \\
\hline $\begin{array}{l}\text { School for } \\
\text { the } \\
\text { Childish }\end{array}$ & SE & $\begin{array}{l}\text { SVT/SVT } \\
\text { Sápmi }\end{array}$ & State media & Maj-Lis Skaltje & 2003 & $30 \mathrm{~min}$ & $\begin{array}{l}\text { TV } \\
\text { Producti } \\
\text { on }\end{array}$ \\
\hline
\end{tabular}




\begin{tabular}{|c|c|c|c|c|c|c|c|}
\hline Savagas & & & & & & & \\
\hline $\begin{array}{l}\text { Sincere } \\
\text { Intentions }\end{array}$ & FI & $\begin{array}{l}\text { Tampereen } \\
\text { ammattikorke } \\
\text { akoulu } \\
\text { TAMK/Taide } \\
\text { ja viestin }\end{array}$ & Educational & Katja Gauriloff & 2004 & $26 \mathrm{~min}$ & Short \\
\hline Sister & FI & $\begin{array}{l}\text { Saamelaisalu } \\
\text { een } \\
\text { koulutuskesk } \\
\text { us SAKK }\end{array}$ & Educational & $\begin{array}{l}\text { Ritva Torikka- } \\
\text { Gelencser }\end{array}$ & 2005 & $14 \mathrm{~min}$ & Short \\
\hline $\begin{array}{l}\text { Sister } \\
\text { Eija: } \\
\text { Caring For } \\
\text { People }\end{array}$ & FI & $\begin{array}{l}\text { Ima Filbma - } \\
\text { ja satneduodji }\end{array}$ & $\begin{array}{l}\text { Private } \\
\text { company }\end{array}$ & Suvi West & 2009 & $29 \min$ & Short \\
\hline $\begin{array}{l}\text { Skierri - } \\
\text { Land of } \\
\text { the Dwarf } \\
\text { Birches }\end{array}$ & FI/SE & $\begin{array}{l}\text { Giron-filmi } \\
\text { Oy }\end{array}$ & $\begin{array}{l}\text { Private } \\
\text { company }\end{array}$ & $\begin{array}{l}\text { Markku } \\
\text { Lehmuskallio }\end{array}$ & 1982 & $\begin{array}{l}1 \mathrm{~h} 58 \\
\min \end{array}$ & Feature \\
\hline Smierru & NO & $\begin{array}{l}\text { The Nordic } \\
\text { Sámi Institute }\end{array}$ & Educational & $\begin{array}{l}\text { Beate Mortensen } \\
\text { Nesheim }\end{array}$ & 2005 & $32 \mathrm{~min}$ & $\begin{array}{l}\text { Docume } \\
\text { ntary }\end{array}$ \\
\hline $\begin{array}{l}\text { Snow } \\
\text { Theatre }\end{array}$ & FI & $\begin{array}{l}\text { Yle Sámi } \\
\text { Radio }\end{array}$ & State media & $\begin{array}{l}\text { Anneli } \\
\text { Lappalainen }\end{array}$ & 2008 & $3 \min$ & $\begin{array}{l}\text { Children' } \\
\text { s Film }\end{array}$ \\
\hline So It & SE & Eva Nutti & Individual & Eva Nutti & 2005 & $5 \mathrm{~min}$ & Short \\
\hline
\end{tabular}




\begin{tabular}{|c|c|c|c|c|c|c|c|}
\hline Should Be & & & & & & & \\
\hline Somby & FI & Sogsakk & Educational & Erkka Haavisto & 2008 & $4 \min$ & Short \\
\hline $\begin{array}{l}\text { Soria } \\
\text { Moria }\end{array}$ & NO & Siivet AS & $\begin{array}{l}\text { Private } \\
\text { company }\end{array}$ & $\begin{array}{l}\text { Anstein } \\
\text { Mikkelsen, } \\
\text { Monica Iren } \\
\text { Hansen }\end{array}$ & 2010 & $28 \mathrm{~min}$ & $\begin{array}{l}\text { Docume } \\
\text { ntary }\end{array}$ \\
\hline $\begin{array}{l}\text { Spiritual } \\
\text { Fashion }\end{array}$ & NO & $\begin{array}{l}\text { NRK/Sámi } \\
\text { Radio }\end{array}$ & State media & $\begin{array}{l}\text { Dan Robert } \\
\text { Larsen }\end{array}$ & 2008 & $30 \mathrm{~min}$ & $\begin{array}{l}\text { Docume } \\
\text { ntary }\end{array}$ \\
\hline Star & FI & Sogsakk & Educational & Kevin Francett & 2011 & $3 \min$ & Short \\
\hline $\begin{array}{l}\text { Successful } \\
\text { ly Lucky } \\
\text { Day (For a } \\
\text { Sámi) }\end{array}$ & SE & $\begin{array}{l}\text { Nordland } \\
\text { College of } \\
\text { Art and Film, } \\
\text { IdiStudios }\end{array}$ & $\begin{array}{l}\text { Mixed } \\
\text { (educational } \\
\text {, private } \\
\text { company) }\end{array}$ & $\begin{array}{l}\text { Per-Josef } \\
\text { Idivuoma, Marja } \\
\text { Bål Nango, } \\
\text { Daniel Bianchini, } \\
\text { Vidar Wikran }\end{array}$ & 2011 & $5 \min$ & Short \\
\hline $\begin{array}{l}\text { Suddenly } \\
\text { Sámi }\end{array}$ & $\mathrm{NO}$ & $\begin{array}{l}\text { Ellen-Astri } \\
\text { Lundby }\end{array}$ & Individual & $\begin{array}{l}\text { Ellen-Astri } \\
\text { Lundby }\end{array}$ & 2009 & $52 \mathrm{~min}$ & $\begin{array}{l}\text { Docume } \\
\text { ntary }\end{array}$ \\
\hline Suhtadit & $\mathrm{NO}$ & $\begin{array}{l}\text { Crazevill } \\
\text { Records }\end{array}$ & $\begin{array}{l}\text { Music } \\
\text { Label }\end{array}$ & Egil Pedersen & 2012 & $4 \min$ & Short \\
\hline $\begin{array}{l}\text { Sverre's } \\
\text { Roots }\end{array}$ & NO & Siivet AS & $\begin{array}{l}\text { Private } \\
\text { company }\end{array}$ & $\begin{array}{l}\text { Anstein } \\
\text { Mikkelsen }\end{array}$ & 2010 & $28 \mathrm{~min}$ & $\begin{array}{l}\text { Docume } \\
\text { ntary }\end{array}$ \\
\hline $\begin{array}{l}\text { Swift and } \\
\text { Energetic }\end{array}$ & & NRK Sápmi & State media & $\begin{array}{l}\text { Nils John } \\
\text { Porsanger }\end{array}$ & 2013 & 29 min & $\begin{array}{l}\text { TV } \\
\text { Producti } \\
\text { on }\end{array}$ \\
\hline
\end{tabular}




\begin{tabular}{|c|c|c|c|c|c|c|c|}
\hline $\begin{array}{l}\text { Taming a } \\
\text { Reindeer }\end{array}$ & FI & Sogsakk & Educational & $\begin{array}{l}\text { Anneli } \\
\text { Lappalainen }\end{array}$ & 2008 & $5 \mathrm{~min}$ & Short \\
\hline $\begin{array}{l}\text { The } \\
\text { Abstract } \\
\text { Ones }\end{array}$ & $\mathrm{NO}$ & $\begin{array}{l}\text { Nordland } \\
\text { College of } \\
\text { Art and Film }\end{array}$ & Educational & Marja Bål Nango & 2011 & $5 \mathrm{~min}$ & Short \\
\hline $\begin{array}{l}\text { The } \\
\text { Awakenin } \\
\text { g }\end{array}$ & FI & $\begin{array}{l}\text { Saamelaisalu } \\
\text { een } \\
\text { koulutuskesk } \\
\text { us SAKK }\end{array}$ & Educational & Matti Kukkola & 2011 & $6 \mathrm{~min}$ & Short \\
\hline The Bear & FI & $\begin{array}{l}\text { Cinemaker } \\
\text { Oy }\end{array}$ & $\begin{array}{l}\text { Private } \\
\text { company }\end{array}$ & $\begin{array}{l}\text { Paul-Anders } \\
\text { Simma }\end{array}$ & 1994 & $27 \mathrm{~min}$ & Short \\
\hline $\begin{array}{l}\text { The Bear } \\
\text { Man }\end{array}$ & $\mathrm{NO}$ & NRK Sápmi & State media & $\begin{array}{l}\text { Nils John } \\
\text { Porsanger, Eilif } \\
\text { Aslaksen }\end{array}$ & 2013 & $39 \mathrm{~min}$ & $\begin{array}{l}\text { Docume } \\
\text { ntary }\end{array}$ \\
\hline $\begin{array}{l}\text { The Best } \\
\text { Clothing }\end{array}$ & FI & Sogsakk & Educational & $\begin{array}{l}\text { Anneli } \\
\text { Lappalainen }\end{array}$ & 2008 & $3 \min$ & Short \\
\hline $\begin{array}{l}\text { The Big } \\
\text { White One }\end{array}$ & FI & Sogsakk & Educational & $\begin{array}{l}\text { Anneli } \\
\text { Lappalainen }\end{array}$ & 2007 & $4 \min$ & Short \\
\hline $\begin{array}{l}\text { The Bird } \\
\text { of Light }\end{array}$ & $\mathrm{NO}$ & $\begin{array}{l}\text { NRK/Sámi } \\
\text { Radio }\end{array}$ & Educational & $\begin{array}{l}\text { Liv-Inger Somby, } \\
\text { Hans-Olov } \\
\text { Landsverk }\end{array}$ & 2010 & $49 \mathrm{~min}$ & $\begin{array}{l}\text { Docume } \\
\text { ntary }\end{array}$ \\
\hline $\begin{array}{l}\text { The } \\
\text { Blizzard }\end{array}$ & $\mathrm{NO}$ & IdiStudios & $\begin{array}{l}\text { Private } \\
\text { company }\end{array}$ & $\begin{array}{l}\text { Per-Josef } \\
\text { Idivuoma }\end{array}$ & 2013 & $5 \mathrm{~min}$ & Short \\
\hline
\end{tabular}




\begin{tabular}{|c|c|c|c|c|c|c|c|}
\hline Whistles & & & & & & & \\
\hline $\begin{array}{l}\text { The } \\
\text { Brooch }\end{array}$ & FI & $\begin{array}{l}\text { Saamelaisalu } \\
\text { een } \\
\text { koulutuskesk } \\
\text { us SAKK }\end{array}$ & Educational & Marjo Ulkuniemi & 2012 & $\begin{array}{l}21 \mathrm{~min} \\
31 \mathrm{sec}\end{array}$ & $\begin{array}{l}\text { Docume } \\
\text { ntary }\end{array}$ \\
\hline $\begin{array}{l}\text { The } \\
\text { Brother } \\
\text { Asks }\end{array}$ & SE & $\begin{array}{l}\text { Bauta Film } \\
\mathrm{AB}\end{array}$ & $\begin{array}{l}\text { Private } \\
\text { company }\end{array}$ & Oskar Östergren & 2014 & $4 \min$ & Short \\
\hline $\begin{array}{l}\text { The Busy } \\
\text { World of } \\
\text { Aaslak }\end{array}$ & FI & Sogsakk & Educational & Marianne Aikio & 2009 & $7 \min$ & Short \\
\hline $\begin{array}{l}\text { The } \\
\text { Career } \\
\text { Woman } \\
\text { From the } \\
\text { Tundra }\end{array}$ & NO & $\begin{array}{l}\text { Polarfilm ja } \\
\text { TV }\end{array}$ & Unknown & Stine Sand Eira & 2005 & $25 \mathrm{~min}$ & $\begin{array}{l}\text { Docume } \\
\text { ntary }\end{array}$ \\
\hline $\begin{array}{l}\text { The } \\
\text { Chamber } \\
\text { maid }\end{array}$ & $\mathrm{SE}$ & $\begin{array}{l}\text { Den Norske } \\
\text { Filmskolen, } \\
\text { Åshild } \\
\text { Ariane } \\
\text { Ramborg }\end{array}$ & $\begin{array}{l}\text { Mixed } \\
\text { (individual, } \\
\text { educational) }\end{array}$ & Ann Holmgren & 2008 & $23 \mathrm{~min}$ & Short \\
\hline $\begin{array}{l}\text { The } \\
\text { Christmas }\end{array}$ & $\mathrm{NO}$ & $\begin{array}{l}\text { Nordland } \\
\text { College of }\end{array}$ & Educational & Marja Bål Nango & 2011 & $\begin{array}{l}13 \mathrm{~min} \\
30 \mathrm{sec}\end{array}$ & $\begin{array}{l}\text { Children' } \\
\text { s Film }\end{array}$ \\
\hline
\end{tabular}




\begin{tabular}{|c|c|c|c|c|c|c|c|}
\hline Troll & & Art and Film & & & & & \\
\hline $\begin{array}{l}\text { The } \\
\text { Christmas } \\
\text { Troll }\end{array}$ & NO & $\begin{array}{l}\text { Nordland } \\
\text { College of } \\
\text { Art and Film }\end{array}$ & Educational & Marja Bål Nango & 2012 & $12 \mathrm{~min}$ & Short \\
\hline $\begin{array}{l}\text { The } \\
\text { Colonial } \\
\text { Gaze Sámi } \\
\text { Artists } \\
\text { Collective }\end{array}$ & NO & $\begin{array}{l}\text { Alexandra } \\
\text { Wingate }\end{array}$ & Individual & $\begin{array}{l}\text { Marja Bål Nango, } \\
\text { Elle-Maija } \\
\text { Tailfeathers }\end{array}$ & 2012 & $15 \mathrm{~min}$ & Short \\
\hline $\begin{array}{l}\text { The Color } \\
\text { of Pain }\end{array}$ & FI & Sogsakk & Educational & Marianne Aikio & 2010 & $4 \mathrm{~min}$ & Short \\
\hline $\begin{array}{l}\text { The } \\
\text { Colourful } \\
\text { Life of } \\
\text { Sámi } \\
\text { Children }\end{array}$ & $\mathrm{NO}$ & & Unknown & $\begin{array}{l}\text { Anne Merete } \\
\text { Gaup }\end{array}$ & 2012 & $4 \min$ & Short \\
\hline $\begin{array}{l}\text { The } \\
\text { Forgotten } \\
\text { Place }\end{array}$ & $\mathrm{NO}$ & Gjert Rognli & Individual & Gjert Rognli & 2009 & $10 \mathrm{~min}$ & Short \\
\hline $\begin{array}{l}\text { The Frog } \\
\text { and the } \\
\text { Owl }\end{array}$ & FI & Sogsakk & Educational & Camilla Arjasmaa & 2011 & $1 \mathrm{~min}$ & Short \\
\hline The Girl & & & Unknown & Ann Holmgren & 2008 & $6 \mathrm{~min}$ & Short \\
\hline
\end{tabular}




\begin{tabular}{|c|c|c|c|c|c|c|c|}
\hline $\begin{array}{l}\text { Who Lost } \\
\text { Her } \\
\text { Dreams }\end{array}$ & & & & & & & \\
\hline $\begin{array}{l}\text { The Girls } \\
\text { of Sevetti }\end{array}$ & $\begin{array}{l}\text { FI } \\
\text { (Sápm } \\
\text { i) }\end{array}$ & $\begin{array}{l}\text { Liisa } \\
\text { Holmberg }\end{array}$ & Individual & Pauliina Fedoroff & 2007 & $2 \min$ & Short \\
\hline $\begin{array}{l}\text { The } \\
\text { Hardest of } \\
\text { These is } \\
\text { Love }\end{array}$ & FI & $\begin{array}{l}\text { Saamelaisalu } \\
\text { een } \\
\text { koulutuskesk } \\
\text { us SAKK }\end{array}$ & Educational & Suvi West & 2005 & $25 \mathrm{~min}$ & $\begin{array}{l}\text { Docume } \\
\text { ntary }\end{array}$ \\
\hline $\begin{array}{l}\text { The } \\
\text { Kautokein } \\
\text { o } \\
\text { Rebellion }\end{array}$ & $\begin{array}{l}\mathrm{NO} / \mathrm{S} \\
\mathrm{E} / \mathrm{DK}\end{array}$ & $\begin{array}{l}\text { Rubicon TV } \\
\text { AS }\end{array}$ & $\begin{array}{l}\text { Private } \\
\text { company }\end{array}$ & Nils Gaup & 2008 & $\begin{array}{l}1 \mathrm{~h} 37 \\
\min \end{array}$ & $\begin{array}{l}\text { Docume } \\
\text { ntary }\end{array}$ \\
\hline $\begin{array}{l}\text { The } \\
\text { Kidnapped }\end{array}$ & NO & $\begin{array}{l}\text { Ninne Film } \\
\text { AS }\end{array}$ & $\begin{array}{l}\text { Private } \\
\text { company }\end{array}$ & $\begin{array}{l}\text { Nils John } \\
\text { Porsanger, Eilif } \\
\text { Aslaksen }\end{array}$ & 2002 & $30 \mathrm{~min}$ & $\begin{array}{l}\text { Docume } \\
\text { ntary }\end{array}$ \\
\hline $\begin{array}{l}\text { The } \\
\text { Language } \\
\text { of my } \\
\text { Heart }\end{array}$ & $\mathrm{NO}$ & $\begin{array}{l}\text { University of } \\
\text { Troms } \emptyset\end{array}$ & Educational & Sirkka Seljevold & 2007 & $36 \mathrm{~min}$ & $\begin{array}{l}\text { Docume } \\
\text { ntary }\end{array}$ \\
\hline $\begin{array}{l}\text { The Last } \\
\text { Day of His }\end{array}$ & FI & $\begin{array}{l}\text { Tampereen } \\
\text { ammattikorke }\end{array}$ & Educational & Katja Gauriloff & 2003 & $7 \mathrm{~min}$ & Short \\
\hline
\end{tabular}




\begin{tabular}{|c|c|c|c|c|c|c|c|}
\hline Life & & $\begin{array}{l}\text { akoulu } \\
\text { TAMK/Taide } \\
\text { ja viestin }\end{array}$ & & & & & \\
\hline $\begin{array}{l}\text { The Last } \\
\text { Night of } \\
\text { Jänispää } \\
\text { Fell }\end{array}$ & FI & Sogsakk & Educational & Ulpu Siponen & 2007 & $5 \mathrm{~min}$ & Short \\
\hline $\begin{array}{l}\text { The Last } \\
\text { Nomad? }\end{array}$ & $\mathrm{NO}$ & Siivet AS & $\begin{array}{l}\text { Private } \\
\text { company }\end{array}$ & Alf Johanssen & 2013 & $50 \mathrm{~min}$ & Feature \\
\hline $\begin{array}{l}\text { The Last } \\
\text { Winter }\end{array}$ & $\mathrm{NO}$ & $\begin{array}{l}\text { NRK/Sámi } \\
\text { Radio }\end{array}$ & State media & Ole Rune Heatta & 2005 & $53 \mathrm{~min}$ & $\begin{array}{l}\text { Docume } \\
\text { ntary }\end{array}$ \\
\hline $\begin{array}{l}\text { The Lost } \\
\text { Land of } \\
\text { the Sámi }\end{array}$ & FI & Yle TV 1 & & $\begin{array}{l}\text { Pauliina Fedoroff, } \\
\text { Peter Launo }\end{array}$ & 2003 & $30 \mathrm{~min}$ & $\begin{array}{l}\text { Docume } \\
\text { ntary }\end{array}$ \\
\hline $\begin{array}{l}\text { The } \\
\text { Minister } \\
\text { of State }\end{array}$ & $\begin{array}{l}\mathrm{FI} / \mathrm{NO} \\
/ \mathrm{SE}\end{array}$ & $\begin{array}{l}\text { Filmpool } \\
\text { Nord } \\
\text { Yleisradio } \\
\text { Oy } \\
\text { Northen } \\
\text { Lights AS } \\
\text { Kinotuotanto } \\
\text { Oy } \\
\text { MovieMaker }\end{array}$ & $\begin{array}{l}\text { Mixed } \\
\text { (private } \\
\text { company, } \\
\text { state media) }\end{array}$ & $\begin{array}{l}\text { Paul-Anders } \\
\text { Simma }\end{array}$ & 1997 & $\begin{array}{l}1 \mathrm{~h} 25 \\
\min \end{array}$ & Feature \\
\hline
\end{tabular}




\begin{tabular}{|c|c|c|c|c|c|c|c|}
\hline & & $\begin{array}{l}\text { s Sweden Ab } \\
\text { Eurimages } \\
\text { Nordisk film- } \\
\text { \& TV-fond } \\
\text { SVT Drama } \\
\text { Svenska } \\
\text { filminstitutet }\end{array}$ & & & & & \\
\hline $\begin{array}{l}\text { The Only } \\
\text { Image of } \\
\text { My Father }\end{array}$ & SE & $\begin{array}{l}\text { Film Ateljén, } \\
\text { Hans-Olov } \\
\text { Utsi AB }\end{array}$ & $\begin{array}{l}\text { Private } \\
\text { companies }\end{array}$ & Kine Boman & 2003 & $58 \mathrm{~min}$ & Short \\
\hline $\begin{array}{l}\text { The Peace } \\
\text { of the } \\
\text { Lemmings }\end{array}$ & FI & $\begin{array}{l}\text { Inari Sámi } \\
\text { Language } \\
\text { Society }\end{array}$ & Non-profit & Suvi Kivelä & 2011 & $4 \mathrm{~min}$ & Short \\
\hline $\begin{array}{l}\text { The Power } \\
\text { of the } \\
\text { River }\end{array}$ & FI & $\begin{array}{l}\text { Saamelaisalu } \\
\text { een } \\
\text { koulutuskesk } \\
\text { us SAKK }\end{array}$ & Educational & $\begin{array}{l}\text { Ima Aikio- } \\
\text { Arianaick }\end{array}$ & 2004 & $11 \mathrm{~min}$ & $\begin{array}{l}\text { Children' } \\
\text { s Film }\end{array}$ \\
\hline $\begin{array}{l}\text { The Right } \\
\text { to the } \\
\text { Land and } \\
\text { the Water }\end{array}$ & $\mathrm{NO}$ & $\begin{array}{l}\text { Sámi Art } \\
\text { Festival }\end{array}$ & Unknown & Geir Tore Holm & 2010 & $13 \mathrm{~min}$ & Short \\
\hline $\begin{array}{l}\text { The Roots } \\
\text { of the Sky }\end{array}$ & FI & $\begin{array}{l}\text { Saamelaisalu } \\
\text { een }\end{array}$ & Educational & Susanna Rauno & 2012 & $6 \min$ & Short \\
\hline
\end{tabular}




\begin{tabular}{|c|c|c|c|c|c|c|c|}
\hline & & $\begin{array}{l}\text { koulutuskesk } \\
\text { us Sogsakk }\end{array}$ & & & & & \\
\hline $\begin{array}{l}\text { The Sámi } \\
\text { Alphabet }\end{array}$ & FI & Sogsakk & Educational & Heli Ahola & 2008 & $2 \min$ & Short \\
\hline $\begin{array}{l}\text { The Sámi } \\
\text { Church } \\
\text { Days in } \\
\text { Inari }\end{array}$ & $\mathrm{NO}$ & & Unknown & $\begin{array}{l}\text { Elle Sofe } \\
\text { Henriksen }\end{array}$ & 2010 & $8 \min$ & Short \\
\hline $\begin{array}{l}\text { The Sámi } \\
\text { Headdress }\end{array}$ & FI & Sogsakk & Educational & Eeva Nykänen & 2008 & $4 \min$ & Short \\
\hline $\begin{array}{l}\text { The Sámi: } \\
\text { A Bait for } \\
\text { Luring } \\
\text { Tourists }\end{array}$ & $\mathrm{NO}$ & $\begin{array}{l}\text { NRK/Sámi } \\
\text { Radio }\end{array}$ & State media & Johs. Kalvemo & 1986 & $30 \mathrm{~min}$ & $\begin{array}{l}\text { Docume } \\
\text { ntary }\end{array}$ \\
\hline $\begin{array}{l}\text { The Secret } \\
\text { Helpers }\end{array}$ & NO & $\begin{array}{l}\text { Govas film \& } \\
\text { mediaproduk } \\
\text { sjon AS }\end{array}$ & $\begin{array}{l}\text { Private } \\
\text { company }\end{array}$ & Harry Johansen & 2012 & $59 \mathrm{~min}$ & Feature \\
\hline $\begin{array}{l}\text { The } \\
\text { Secrets of } \\
\text { a } \\
\text { Mountain }\end{array}$ & $\mathrm{NO}$ & $\begin{array}{l}\text { NRK/Sámi } \\
\text { Radio }\end{array}$ & State media & Thoralf Balto & 2010 & $28 \mathrm{~min}$ & $\begin{array}{l}\text { Docume } \\
\text { ntary }\end{array}$ \\
\hline $\begin{array}{l}\text { The Son } \\
\text { of the Sun }\end{array}$ & FI & Sogsakk & Educational & Pälvi Magga & 2008 & $3 \min$ & Short \\
\hline
\end{tabular}




\begin{tabular}{|c|c|c|c|c|c|c|c|c|}
\hline $\begin{array}{l}\text { The Sound } \\
\text { of Calves } \\
\text { in the } \\
\text { Wind }\end{array}$ & FI & \multicolumn{2}{|c|}{ Sogsakk } & Educational & Anne Lantto & 2003 & $8 \mathrm{~min}$ & Short \\
\hline $\begin{array}{l}\text { The } \\
\text { Spiritual } \\
\text { Kiss }\end{array}$ & $\mathrm{NO}$ & & & Unknown & Gjert Rognli & 2009 & $5 \mathrm{~min}$ & Short \\
\hline $\begin{array}{l}\text { The Story } \\
\text { of Arctic } \\
\text { Love }\end{array}$ & & $\begin{array}{l}\text { Firstfiln } \\
\text { Televisi } \\
\text { AB }\end{array}$ & & $\begin{array}{l}\text { Private } \\
\text { company }\end{array}$ & $\begin{array}{l}\text { Paul-Anders } \\
\text { Simma }\end{array}$ & 2000 & $30 \mathrm{~min}$ & Short \\
\hline $\begin{array}{l}\text { The Story } \\
\text { of Johan } \\
\text { Turi }\end{array}$ & $\mathrm{SE}$ & SVT & & State media & Maj-Lis Skaltje & 2005 & $45 \mathrm{~min}$ & Short \\
\hline $\begin{array}{l}\text { The } \\
\text { Taking of } \\
\text { Sámiland }\end{array}$ & $\mathrm{NO}$ & $\begin{array}{l}\text { Davvi } \\
\text { Media } \\
\text { /Vara } \\
\text { ng } \\
\text { film }\end{array}$ & Pri & e companies & $\begin{array}{l}\text { Skule Eriksen, } \\
\text { Kåre Tannvik }\end{array}$ & 1983 & $30 \mathrm{~min}$ & $\begin{array}{l}\text { Docume } \\
\text { ntary }\end{array}$ \\
\hline $\begin{array}{l}\text { The Trout } \\
\text { Gets } \\
\text { Angry }\end{array}$ & FI & $\begin{array}{l}\text { Ima } \\
\text { Filbma- } \\
\text { ja } \\
\text { sátnedu } \\
\text { dji }\end{array}$ & & $\begin{array}{l}\text { xed (private } \\
\text { npany, non- } \\
\text { fit) }\end{array}$ & $\begin{array}{l}\text { Ima Aikio- } \\
\text { Airianack }\end{array}$ & 2013 & $4 \min$ & Short \\
\hline
\end{tabular}




\begin{tabular}{|c|c|c|c|c|c|c|c|}
\hline & & $\begin{array}{l}\text { Inarin } \\
\text { Elokuvat } \\
\text { eollisuus }\end{array}$ & & & & & \\
\hline $\begin{array}{l}\text { The } \\
\text { Tundra } \\
\text { Settlers }\end{array}$ & NO & $\begin{array}{l}\text { Filbmag } \\
\text { oahti AS }\end{array}$ & Private company & Anne Lajla Utsi & 2004 & $52 \mathrm{~min}$ & $\begin{array}{l}\text { Docume } \\
\text { ntary }\end{array}$ \\
\hline $\begin{array}{l}\text { The Wild } \\
\text { Tundra }\end{array}$ & $\mathrm{NO}$ & $\begin{array}{l}\text { Kautokei } \\
\text { no Film } \\
\text { AS, ISF } \\
\text { AS }\end{array}$ & $\begin{array}{l}\text { Mixed (non- } \\
\text { government, } \\
\text { private } \\
\text { company) }\end{array}$ & $\begin{array}{l}\text { Anne Merete A. } \\
\text { Gaup } \\
\text { Johan Ante Utsi } \\
\text { Inga Marie Risvik } \\
\text { Elle Marja P. Eira } \\
\text { Yvonne } \\
\text { Thommassen } \\
\text { Håkon Isak Vars }\end{array}$ & 2009 & $6 \mathrm{~min}$ & Short \\
\hline $\begin{array}{l}\text { The Wind } \\
\text { Whispers } \\
\text { There Is } \\
\text { Someone } \\
\text { Behind the } \\
\text { Tundra }\end{array}$ & $\mathrm{NO}$ & $\begin{array}{l}\text { KABong } \\
\text { o } \\
\text { Producti } \\
\text { ons }\end{array}$ & $\begin{array}{l}\text { Private } \\
\text { Company }\end{array}$ & $\begin{array}{l}\text { Ken Are Bongo, } \\
\text { Elle Sofe } \\
\text { Henriksen }\end{array}$ & 2006 & $10 \mathrm{~min}$ & Short \\
\hline $\begin{array}{l}\text { The } \\
\text { Yoiking } \\
\text { Hand }\end{array}$ & $\mathrm{NO}$ & $\begin{array}{l}\text { Kautokei } \\
\text { no Film } \\
\text { AS, ISF }\end{array}$ & $\begin{array}{l}\text { Mixed (non- } \\
\text { government, } \\
\text { private }\end{array}$ & $\begin{array}{l}\text { Elle Sofe } \\
\text { Henriksen }\end{array}$ & 2011 & $5 \mathrm{~min}$ & Short \\
\hline
\end{tabular}




\begin{tabular}{|c|c|c|c|c|c|c|c|}
\hline & & AS & company) & & & & \\
\hline $\begin{array}{l}\text { Thoughts } \\
\text { of the } \\
\text { Moment }\end{array}$ & FI & Sogsakk & Educational & Janne Kyrö & 2011 & $\begin{array}{l}2 \min 10 \\
\sec \end{array}$ & Short \\
\hline Threats & $\mathrm{NO}$ & NKFS & Educational & $\begin{array}{l}\text { Anne Merete } \\
\text { Gaup }\end{array}$ & 2013 & $11 \mathrm{~min}$ & Short \\
\hline $\begin{array}{l}\text { Three } \\
\text { Reindeer } \\
\text { and One } \\
\text { Apartment }\end{array}$ & $\mathrm{NO}$ & $\begin{array}{l}\text { NRK, } \\
\text { Bengt } \\
\text { Roger } \\
\text { Kåven }\end{array}$ & $\begin{array}{l}\text { Mixed (state } \\
\text { media, } \\
\text { individual) }\end{array}$ & $\begin{array}{l}\text { Wenche Marie } \\
\text { Haetta }\end{array}$ & 2009 & $29 \mathrm{~min}$ & $\begin{array}{l}\text { TV } \\
\text { Producti } \\
\text { on }\end{array}$ \\
\hline $\begin{array}{l}\text { Thrown } \\
\text { on Land }\end{array}$ & $\mathrm{NO}$ & NRK & State media & Harry Johansen & 2009 & $45 \mathrm{~min}$ & $\begin{array}{l}\text { Docume } \\
\text { ntary }\end{array}$ \\
\hline Time & $\mathrm{NO}$ & & Unknown & $\begin{array}{l}\text { Niels Ovlla } \\
\text { Dunfjell, Ante } \\
\text { Mahtte Eira }\end{array}$ & 2013 & $3 \min$ & Short \\
\hline Time & FI & Sogsakk & Educational & Sunna Kitti & 2010 & $1 \mathrm{~min}$ & Short \\
\hline $\begin{array}{l}\text { Time } \\
\text { Changes, } \\
\text { Habits Not }\end{array}$ & FI & $\begin{array}{l}\text { Samedig } \\
\text { gi, } \\
\text { Sogsakk, } \\
\text { theonemi } \\
\text { nutesJr. }\end{array}$ & $\begin{array}{l}\text { Mixed (non- } \\
\text { profit, } \\
\text { educational) }\end{array}$ & Salla-Mari West & 2006 & $1 \mathrm{~min}$ & Short \\
\hline $\begin{array}{l}\text { To Herd } \\
\text { Reindeer }\end{array}$ & $\mathrm{NO}$ & $\begin{array}{l}\text { Kautokei } \\
\text { no Film }\end{array}$ & Private company & $\begin{array}{l}\text { Ken Are Bongo, } \\
\text { Elle-Marja P. Eira }\end{array}$ & 2012 & $\begin{array}{l}6 \min 22 \\
\sec \end{array}$ & Short \\
\hline
\end{tabular}




\begin{tabular}{|c|c|c|c|c|c|c|c|}
\hline & & AS & & & & & \\
\hline $\begin{array}{l}\text { To the } \\
\text { End of the } \\
\text { Line }\end{array}$ & $\mathrm{NO}$ & $\begin{array}{l}\text { Norsk } \\
\text { Telemus } \\
\text { eum }\end{array}$ & Non-profit & $\begin{array}{l}\text { Nils John } \\
\text { Porsanger }\end{array}$ & 2006 & $25 \mathrm{~min}$ & Short \\
\hline Totem & $\begin{array}{l}\mathrm{SE} / \mathrm{C} \\
\mathrm{O}\end{array}$ & & Unknown & $\begin{array}{l}\text { Bjarne Store- } \\
\text { Jakobsen }\end{array}$ & 2002 & $27 \mathrm{~min}$ & $\begin{array}{l}\text { Docume } \\
\text { ntary }\end{array}$ \\
\hline $\begin{array}{l}\text { Trackback } \\
\text { - A } \\
\text { Lappish } \\
\text { Soul }\end{array}$ & $\mathrm{SE}$ & $\begin{array}{l}\text { Filmpool } \\
\text { Nord }\end{array}$ & Private company & Anette Niia & 2000 & $30 \mathrm{~min}$ & $\begin{array}{l}\text { Docume } \\
\text { ntary }\end{array}$ \\
\hline Trambo & FI & $\begin{array}{l}\text { Marja } \\
\text { Helander }\end{array}$ & Individual & Marja Helander & 2013 & $4 \min$ & $\begin{array}{l}\text { Docume } \\
\text { ntary }\end{array}$ \\
\hline $\begin{array}{l}\text { Treffan } \\
\text { peaäccam } \\
\text { ne'kk }\end{array}$ & FI & $\begin{array}{l}\text { Yleisradi } \\
\text { o Oy, } \\
\text { TV2, } \\
\text { SVT }\end{array}$ & State media & $\begin{array}{l}\text { Elli Rantala, } \\
\text { Sukio Aikio }\end{array}$ & 1983 & $20 \mathrm{~min}$ & $\begin{array}{l}\text { Docume } \\
\text { ntary }\end{array}$ \\
\hline $\begin{array}{l}\text { Tundra of } \\
\text { Arvas }\end{array}$ & SE & $\begin{array}{l}\text { LittleBig } \\
\text { Producti } \\
\text { ons }\end{array}$ & Private company & Liselotte Wajstedt & 2009 & $\begin{array}{l}3 \min 30 \\
\sec \end{array}$ & Short \\
\hline Tunnel & FI & Sogsakk & Educational & Camilla Arjasmaa & 2011 & $\begin{array}{l}1 \min 40 \\
\sec \end{array}$ & Short \\
\hline Twinkle & FI & Sogsakk & Educational & $\begin{array}{l}\text { Vilma } \\
\text { Rautakangas }\end{array}$ & 2011 & $2 \min$ & Short \\
\hline
\end{tabular}




\begin{tabular}{|c|c|c|c|c|c|c|c|}
\hline $\begin{array}{l}\text { Unplugge } \\
\text { d }\end{array}$ & NO & $\begin{array}{l}\text { Synlig- } \\
\text { Prosjekt, } \\
\text { Finnmar } \\
\text { k }\end{array}$ & Unknown & Ken Are Bongo & 2003 & $3 \min$ & Short \\
\hline $\begin{array}{l}\text { Unto Dust } \\
\text { Thou } \\
\text { Shalt } \\
\text { Return }\end{array}$ & NO & $\begin{array}{l}\text { NRK/Sá } \\
\text { mi Radio }\end{array}$ & State media & Johs. Kalvemo & 1999 & $45 \mathrm{~min}$ & $\begin{array}{l}\text { Docume } \\
\text { ntary }\end{array}$ \\
\hline Urri-Uula & FI & $\begin{array}{l}\text { Unilumi } \\
\text { Oy }\end{array}$ & Private company & $\begin{array}{l}\text { Matti Morottaja, } \\
\text { Aune Kuuva }\end{array}$ & 2001 & $20 \mathrm{~min}$ & Short \\
\hline Uuh! & FI & $\begin{array}{l}\text { Tuupa } \\
\text { Records }\end{array}$ & Music label & Mika Luovi & 2007 & $3 \min$ & Short \\
\hline $\begin{array}{l}\text { Vanishing } \\
\text { Frontier }\end{array}$ & $\begin{array}{l}\text { RU/N } \\
\mathrm{O}\end{array}$ & $\begin{array}{l}\text { Govat } \\
\text { AS }\end{array}$ & Private company & $\begin{array}{l}\text { Nils Gaup, Magne } \\
\text { Ove Varsi }\end{array}$ & 1993 & $\begin{array}{l}22 \mathrm{~min} \\
43 \mathrm{sec}\end{array}$ & $\begin{array}{l}\text { Docume } \\
\text { ntary }\end{array}$ \\
\hline Vehicles & FI & & Unknown & $\begin{array}{l}\text { Ima Aikio- } \\
\text { Airianack }\end{array}$ & 2013 & $3 \min$ & Short \\
\hline Vermin & SE & $\begin{array}{l}\text { Bauta } \\
\text { Film AB, } \\
\text { Lampray }\end{array}$ & $\begin{array}{l}\text { Private } \\
\text { companies }\end{array}$ & $\begin{array}{l}\text { Jonas Ernhill, } \\
\text { Tage Hervén }\end{array}$ & 2013 & $7 \mathrm{~min}$ & $\begin{array}{l}\text { Docume } \\
\text { ntary }\end{array}$ \\
\hline $\begin{array}{l}\text { Voices of } \\
\text { Sápmi }\end{array}$ & FI/UK & & Unknown & Alistair Fowler & 2013 & $58 \mathrm{~min}$ & $\begin{array}{l}\text { Docume } \\
\text { ntary }\end{array}$ \\
\hline $\begin{array}{l}\text { Vuomasel } \\
\text { kä Hill }\end{array}$ & FI & Sogsakk & Educational & Pälvi Magga & 2009 & $\begin{array}{l}19 \mathrm{~min} \\
20 \mathrm{sec}\end{array}$ & Short \\
\hline
\end{tabular}




\begin{tabular}{|c|c|c|c|c|c|c|c|}
\hline $\begin{array}{l}\text { Wanna b } \\
\text { cool }\end{array}$ & NO & ISF AS & $\begin{array}{l}\text { Mixed (non- } \\
\text { government, } \\
\text { multiple funding } \\
\text { sources) }\end{array}$ & $\begin{array}{l}\text { Save Marie } \\
\text { Kvitfjell } \\
\text { Tonje Halonen } \\
\text { Maren Benedicte } \\
\text { Storslett } \\
\text { Anne Merete A. } \\
\text { Gaup } \\
\text { Karianne Aho } \\
\text { Silja Katrine } \\
\text { Hansen }\end{array}$ & 2009 & $6 \mathrm{~min}$ & Short \\
\hline $\begin{array}{l}\text { Warm } \\
\text { Shoes }\end{array}$ & FI & $\begin{array}{l}\text { Inari } \\
\text { Sámi } \\
\text { Languag } \\
\text { e Society }\end{array}$ & Non-profit & $\begin{array}{l}\text { Anneli } \\
\text { Lappalainen }\end{array}$ & 2010 & $15 \mathrm{~min}$ & Short \\
\hline $\begin{array}{l}\text { Wearing } \\
\text { the Sámi } \\
\text { Dress }\end{array}$ & FI & $\begin{array}{l}\text { Sámi } \\
\text { Siida Ry }\end{array}$ & Non-profit & $\begin{array}{l}\text { Ima Aikio } \\
\text { Arianaick }\end{array}$ & 2005 & $\begin{array}{l}3 \min 10 \\
\sec \end{array}$ & Short \\
\hline $\begin{array}{l}\text { What Are } \\
\text { Today's } \\
\text { Young } \\
\text { Like in the } \\
\text { Future? }\end{array}$ & $\mathrm{NO}$ & $\begin{array}{l}\text { Nuoraj } \\
\text { TV }\end{array}$ & Unknown & $\begin{array}{l}\text { Simon Piera } \\
\text { Paulsen }\end{array}$ & 2010 & $2 \min$ & Short \\
\hline What & RU & Sámi & Mixed (private & Sergei Gavrilov & 2013 & $29 \mathrm{~min}$ & Short \\
\hline
\end{tabular}




\begin{tabular}{|c|c|c|c|c|c|c|c|}
\hline Now? & & $\begin{array}{l}\text { Allaskuv } \\
\text { la, } \\
\text { Gavrilov } \\
\text { Media }\end{array}$ & $\begin{array}{l}\text { company, } \\
\text { educational) }\end{array}$ & & & & \\
\hline $\begin{array}{l}\text { When } \\
\text { We're } \\
\text { Born We } \\
\text { Don't } \\
\text { Know } \\
\text { Which } \\
\text { Way } \\
\text { We're } \\
\text { Heading }\end{array}$ & FI & Sogsakk & Educational & Kauko Mustonen & 2007 & $5 \mathrm{~min}$ & Short \\
\hline $\begin{array}{l}\text { Where } \\
\text { Are You, } \\
\text { Grandpa? }\end{array}$ & $\begin{array}{l}\text { NO } \\
\text { (Sápm } \\
\text { i) }\end{array}$ & $\begin{array}{l}\text { NRK } \\
\text { Faktor }\end{array}$ & State media & Suvi West & 2007 & $29 \mathrm{~min}$ & $\begin{array}{l}\text { Docume } \\
\text { ntary }\end{array}$ \\
\hline $\begin{array}{l}\text { Where Do } \\
\text { You Come } \\
\text { From? }\end{array}$ & FI & $\begin{array}{l}\text { Suomen } \\
\text { Televisio }\end{array}$ & Unknown & Kirsti Lindberg & 1963 & $4 \min$ & Short \\
\hline $\begin{array}{l}\text { Wide As } \\
\text { Oceans }\end{array}$ & SE & $\begin{array}{l}\text { Bautafil } \\
\mathrm{m} \mathrm{AB}\end{array}$ & Private company & Oskar Östergren & 2013 & $4 \min$ & $\begin{array}{l}\text { Docume } \\
\text { ntary }\end{array}$ \\
\hline $\begin{array}{l}\text { Wind in } \\
\text { my Heart }\end{array}$ & $\begin{array}{l}\mathrm{NO} / \mathrm{S} \\
\mathrm{E}\end{array}$ & $\begin{array}{l}\text { NRK/Sá } \\
\text { mi }\end{array}$ & State media & $\begin{array}{l}\text { Johs. Kalvemo, } \\
\text { John Erling Utsi }\end{array}$ & 2002 & $30 \mathrm{~min}$ & $\begin{array}{l}\text { Docume } \\
\text { ntary }\end{array}$ \\
\hline
\end{tabular}




\begin{tabular}{|c|c|c|c|c|c|c|c|}
\hline & & $\begin{array}{l}\text { Radio, } \\
\text { SVT } \\
\text { Sápmi }\end{array}$ & & & & & \\
\hline $\begin{array}{l}\text { Wind } \\
\text { Power }\end{array}$ & $\mathrm{NO}$ & $\begin{array}{l}\text { Pomor } \\
\text { Film }\end{array}$ & Private company & Stein Bjorn & 2012 & $52 \mathrm{~min}$ & Feature \\
\hline Winged & FI & $\begin{array}{l}\text { Ima } \\
\text { Filbma- } \\
\text { ja } \\
\text { sátneduo } \\
\text { dji } \\
\text { Inarin } \\
\text { Elokuvat } \\
\text { eollisuus }\end{array}$ & $\begin{array}{l}\text { Mixed (private } \\
\text { company, non- } \\
\text { profit) }\end{array}$ & $\begin{array}{l}\text { Ima Aikio } \\
\text { Arianaick }\end{array}$ & 2012 & $28 \mathrm{~min}$ & $\begin{array}{l}\text { Docume } \\
\text { ntary }\end{array}$ \\
\hline $\begin{array}{l}\text { Woman is } \\
\text { My God }\end{array}$ & $\mathrm{NO}$ & $\begin{array}{l}\text { NRK/Sá } \\
\text { mi Radio }\end{array}$ & State media & Johs. Kalvemo & 2011 & $30 \mathrm{~min}$ & $\begin{array}{l}\text { Docume } \\
\text { ntary }\end{array}$ \\
\hline Yoik & $\mathrm{SE}$ & & Unknown & Maj-Lis Skaltje & 2013 & $84 \mathrm{~min}$ & Feature \\
\hline $\begin{array}{l}\text { Yoik } \\
\text { Fever }\end{array}$ & $\mathrm{NO}$ & & Unknown & $\begin{array}{l}\text { Ellen-Astri } \\
\text { Lundby }\end{array}$ & 2013 & $55 \mathrm{~min}$ & Feature \\
\hline
\end{tabular}


Films Outside of the Sámi Film Database ${ }^{157}$

\begin{tabular}{|c|c|c|c|c|c|c|c|}
\hline Film & Country & Producer & $\begin{array}{l}\text { Type of } \\
\text { Producer }\end{array}$ & Filmmaker & Year & $\begin{array}{l}\text { Runti } \\
\text { me }\end{array}$ & Genre \\
\hline $\begin{array}{l}8 \\
\text { Arstider } \\
158\end{array}$ & $\mathrm{SE}$ & & Unknown & Oskar Östergren & 2014 & $\begin{array}{l}38 \\
\min \end{array}$ & Short \\
\hline $\begin{array}{l}\text { A Bloody } \\
\text { Joik }^{159}\end{array}$ & $\mathrm{SE}$ & & Unknown & Jorun Collin & 2006 & $\begin{array}{l}28 \\
\text { Min }\end{array}$ & \\
\hline $\begin{array}{l}\text { A Soul of } \\
\text { a City }^{160}\end{array}$ & $\mathrm{SE}$ & & Unknown & Liselotte Wajstedt & 2012 & $\begin{array}{l}5 \mathrm{~min} \\
50\end{array}$ & Short \\
\hline $\begin{array}{l}\text { Aftenland } \\
\text { et }^{161}\end{array}$ & $\mathrm{NO}$ & NRK & State media & Erik Poppe & 1994 & $\begin{array}{l}14 \\
\min \end{array}$ & Short \\
\hline $\mathrm{Ahkku}^{162}$ & FI & $\begin{array}{l}\text { Sami } \\
\text { education } \\
\text { institute }\end{array}$ & Educational & Xia Torikka & 2016 & $\begin{array}{l}27 \\
\min \end{array}$ & $\begin{array}{l}\text { Docum } \\
\text { entary }\end{array}$ \\
\hline Ahpi ${ }^{163}$ & $\mathrm{SE}$ & & Unknown & Oskar Östergren & 2013 & $4 \min$ & $\begin{array}{l}\text { Music } \\
\text { Video }\end{array}$ \\
\hline
\end{tabular}

${ }^{157}$ In some cases, I was unsure about the origins of specific films, and whether they could be considered examples of Sámi filmmaking. Any films that appeared ambiguous have been omitted from this section. In addition, some works shown in mixed media exhibitions were simply listed by title, without clarification as to which medium the artist used; in the absence of additional information, these, too, have been omitted.

158 "Dellie Maa Program", Dellie Maa Festival, 2014, digital PDF file courtesy of Oskar Östergren.

159 "Indigenous Peoples' Film Festival, Siida, Inari Jan 24th-28th, 2008", Skábmagovat Film Festival, accessed May 9 2017, http://www.Skábmagovat.fi/Skábma_vanhat/Skábma_2008/ohjelma_en/ohjelma_perjantai.htm.

160 "CV", Liselotte Wajstedt, accessed May 9 2017, http://www.liselottewajstedt.com/bio.

161 "Skábmagovat 2004-05", Skábmagovat Film Festival, accessed May 9 2017, http://www.Skábmagovat.fi/Skábma_vanhat/Skábma_2005/800en.html.

162 "Áhkku", Skábmagovat Film Festival, accessed June 22 2017, http://skabmagovat.fi/skabmagovat_2014/?page_id=7992.

163 "Skábmagovat 2014", Skábmagovat Film Festival, Microsoft Word document courtesy of Kaija Anttonen. 


\begin{tabular}{|c|c|c|c|c|c|c|c|}
\hline $\operatorname{Aigin}^{164}$ & & $\begin{array}{l}\text { Jukka } \\
\text { Vidgren, } \\
\text { Mutant } \\
\text { Koala } \\
\text { Pictures }\end{array}$ & $\begin{array}{l}\text { Mixed } \\
\text { (individual, } \\
\text { private } \\
\text { company) }\end{array}$ & Jouni West & 2014 & $\begin{array}{l}10 \\
\min \end{array}$ & Short \\
\hline $\begin{array}{l}\text { Aile Ja } \\
\text { Ahkku }^{165}\end{array}$ & $\mathrm{NO}$ & Julev Film & $\begin{array}{l}\text { Private } \\
\text { company }\end{array}$ & Silja Somby & 2015 & $\begin{array}{l}12 \\
\min \end{array}$ & Short \\
\hline $\begin{array}{l}\text { Amoc - } \\
\text { Vuosmus } \\
166\end{array}$ & FI & & Unknown & Ima Aikio & 2015 & $\begin{array}{l}30 \\
\min \end{array}$ & $\begin{array}{l}\text { Docum } \\
\text { entary }\end{array}$ \\
\hline $\mathrm{Ara}^{167}$ & $\mathrm{NZ}$ & & Unknown & $\begin{array}{l}\text { Kath Akuhata- } \\
\text { Brown, Sara } \\
\text { Margrethe Oskal, } \\
\text { Echota Kilsnight }\end{array}$ & 2016 & $8 \mathrm{~min}$ & \\
\hline $\begin{array}{l}\text { Assimilat } \\
\text { ion } \\
\text { Blues }^{168}\end{array}$ & FI & $\begin{array}{l}\text { Tuupa } \\
\text { Records }\end{array}$ & Music Label & Eetu Nieminen & 2014 & $3 \mathrm{~min}$ & $\begin{array}{l}\text { Music } \\
\text { Video }\end{array}$ \\
\hline $\begin{array}{l}\text { Att dela } \\
\text { allt }{ }^{169}\end{array}$ & SE & Hannasofia & $\begin{array}{l}\text { Private } \\
\text { company }\end{array}$ & Amanda Kernell & 2009 & & Short \\
\hline
\end{tabular}

164 "Short Films Funded by the International Sámi Film Centre", International Sámi Film Institute, accessed May 9 2017, http://www.isfi.no/eng/films/short.

165 "7 Sámi Films", International Sámi Film Institute, accessed May 9 2017, http://www.isfi.no/eng/films/7Sámistories.

166"Skábmagovat 2016: Kaamoksen Kuvia/Reflections of the Endless Night", Inari: Skábmagovat Film Festival, 2016.

167 "Ara", Skábmagovat Film Festival, accessed June 22 2017, http://skabmagovat.fi/skabmagovat_2014/?page_id=8647

168 "Skábmagovat 2015", Skábmagovat Film Festival, Microsoft Word document courtesy of Kaija Anttonen. 


\begin{tabular}{|c|c|c|c|c|c|c|c|}
\hline $\begin{array}{l}\text { Atte } \\
\text { munnje } \\
\text { sáni } \\
\text { saji }^{170}\end{array}$ & $\mathrm{NO}$ & & Unknown & $\begin{array}{l}\text { Sara Margrethe } \\
\text { Oskal }\end{array}$ & 2016 & & $\begin{array}{l}\text { Music } \\
\text { Video }\end{array}$ \\
\hline $\begin{array}{l}\text { Aurora } \\
\text { Borealis } \\
171\end{array}$ & & & Unknown & Martti Rikonen & & & \\
\hline $\begin{array}{l}\text { Aurora } \\
\text { Mirror }^{172}\end{array}$ & FI & $\begin{array}{l}\text { Sami } \\
\text { education } \\
\text { institute }\end{array}$ & Educational & Tomi Rantonen & 2016 & $3 \mathrm{~min}$ & Short \\
\hline $\begin{array}{l}\text { Axis } 49 \\
\text { Coryphee } \\
173\end{array}$ & SE & & Unknown & Ann Holmgren & 2016 & $6 \mathrm{~min}$ & Short \\
\hline $\mathrm{BBB}^{174}$ & FI & & Unknown & $\begin{array}{l}\text { Ima Aikio- } \\
\text { Arianaick }\end{array}$ & 2013 & $3 \mathrm{~min}$ & Short \\
\hline Bihttos 175 & & Violator & Private & Elle-Maija & 2014 & 14 & Docum \\
\hline
\end{tabular}

169 "Amanda Kernell", Swedish Film Institute Database, accessed May 9 2017, http://www.sfi.se/en-GB/Swedishfilm-

database/Item/?type=PERSON\&itemid=325817\&iv=MOVIE\&ref=\%2ftemplates $\% 2 \mathrm{fS}$ wedishFilmSearchResult.asp $\mathrm{x} \% 3$ fid $\% 3 \mathrm{~d} 1225 \% 26$ epslanguage $\% 3 \mathrm{den}-$

GB $\% 26$ searchword\%3damanda+kernell\%26type $\% 3 \mathrm{dPerson} \% 26 \mathrm{match} \% 3 \mathrm{dBegin} \% 26 \mathrm{page} \% 3 \mathrm{~d} 1 \% 26 \mathrm{prom} \% 3 \mathrm{dFalse}$.

170 "Atte munje sáni saji", Skábmagovat Film Festival, accessed June 22 2017, http://skabmagovat.fi/skabmagovat_2014/?page_id=10351.

171 "Skábmagovat 2003", Skábmagovat Film Festival, accessed June 202017 , http://www.skabmagovat.fi/skabma_vanhat/skabma_2003/800en.html.

172 "Aurora Mirror", Skábmagovat Film Festival, accessed June 22 2017, http://skabmagovat.fi/skabmagovat_2014/?page_id=8344.

173 "Axis 49 Coryphee", Skábmagovat Film Festival, accessed June 22 2017, http://skabmagovat.fi/skabmagovat_2014/?page_id=8530.

174 "Skábmagovat 2014", Skábmagovat Film Festival, Microsoft Word document courtesy of Kaija Anttonen.

175 "Documentary Films Funded by the International Sámi Film Centre", International Sámi Film Institute, accessed May 9 2017, http://www.isfi.no/eng/films/documentary. 


\begin{tabular}{|c|c|c|c|c|c|c|c|}
\hline & & Films & company & Tailfeathers & & $\min$ & entary \\
\hline $\begin{array}{l}\text { Bivdoagi } \\
176\end{array}$ & $\mathrm{FI} / \mathrm{NO}$ & & Unknown & Suohpanterror & 2015 & $7 \min$ & $\begin{array}{l}\text { Music } \\
\text { Video }\end{array}$ \\
\hline $\begin{array}{l}\text { Bloodlan } \\
\mathrm{d}^{177}\end{array}$ & $\mathrm{CA}$ & $\begin{array}{l}\text { Elle-Maija } \\
\text { Tailfeathers }\end{array}$ & Individual & $\begin{array}{l}\text { Elle-Maija } \\
\text { Tailfeathers }\end{array}$ & 2011 & $4 \min$ & $\begin{array}{l}\text { Experi } \\
\text { mental }\end{array}$ \\
\hline $\begin{array}{l}\text { Boaimma } \\
\text { sbardni }^{178}\end{array}$ & & $\begin{array}{l}\text { ISFI, Sámi } \\
\text { University } \\
\text { College }\end{array}$ & $\begin{array}{l}\text { Mixed (non- } \\
\text { govermnent, } \\
\text { educational) }\end{array}$ & & 2013 & $\begin{array}{l}13 \\
\min \end{array}$ & $\begin{array}{l}\mathrm{Tv} \\
\text { product } \\
\text { ion }\end{array}$ \\
\hline $\begin{array}{l}\text { Boazodoa } \\
\text { lli } \\
\text { Calmmiin } \\
\text { guin }^{179}\end{array}$ & FI & & Unknown & Aslak Paltto & 2015 & $\begin{array}{l}20 \\
\min \end{array}$ & $\begin{array}{l}\text { Docum } \\
\text { entary }\end{array}$ \\
\hline $\begin{array}{l}\text { Boazolih } \\
\mathrm{kku}^{180}\end{array}$ & $\mathrm{NO}$ & NRK & State media & Gunnar Hammer & 2008 & $\begin{array}{l}40 \\
\min \end{array}$ & \\
\hline $\begin{array}{l}\text { Boazzova } \\
\text { zzit }^{181}\end{array}$ & SE & $\begin{array}{l}\text { SVT/Per- } \\
\text { Josef } \\
\text { Idivuoma }\end{array}$ & Unknown & August Sandström & 2016 & $\begin{array}{l}30 \\
\min \end{array}$ & $\begin{array}{l}\text { Docum } \\
\text { entary }\end{array}$ \\
\hline
\end{tabular}

176 "Skábmagovat 2016: Kaamoksen Kuvia/Reflections of the Endless Night", Inari: Skábmagovat Film Festival, 2016.

177 "Film and Video Works", Elle-Maija Tailfeathers.com, accessed May 9 2017, http://elle-maijatailfeathers.com/?page_id=311.

178 "Short Films Funded by the International Sámi Film Centre", International Sámi Film Institute, accessed May 9 2017, http://www.isfi.no/eng/films/short.

179 "Skábmagovat 2016: Kaamoksen Kuvia/Reflections of the Endless Night", Inari: Skábmagovat Film Festival, 2016.

180 "Skábmagovat 2008", Skábmagovat Film Festival, accessed May 9 2017,

http://www.Skábmagovat.fi/Skábma_vanhat/Skábma_2008/ohjelma_en/ohjelmainfo_su.htm\#.

181 "Boazzovazzit", Skábmagovat Film Festival, accessed June 22 2017,

http://skabmagovat.fi/skabmagovat_2014/?page_id=8530. 


\begin{tabular}{|c|c|c|c|c|c|c|c|}
\hline Bonki $^{182}$ & & $\begin{array}{l}\text { Odd Levi } \\
\text { Paulsen, } \\
\text { Julev Film }\end{array}$ & Unknown & Silja Somby & 2014 & $\begin{array}{l}19 \\
\min \end{array}$ & Short \\
\hline $\begin{array}{l}\text { Burn the } \\
\text { Drums }{ }^{183}\end{array}$ & SE & & Unknown & Liselotte Wajstedt & 2016 & $\begin{array}{l}2 \min \\
4 \\
\text { secon } \\
\text { ds }\end{array}$ & Short \\
\hline $\begin{array}{l}\text { Calbmera } \\
\text { vkaleame } \\
\mathrm{s}^{184}\end{array}$ & $\mathrm{NO}$ & & Unknown & Jan Helmer Olson & 2016 & $6 \mathrm{~min}$ & Short \\
\hline $\begin{array}{l}\text { Ceahppi } \\
185\end{array}$ & FI & $\begin{array}{l}\text { Sámi } \\
\text { educational } \\
\text { centre }\end{array}$ & Educational & & & & $\begin{array}{l}\text { Docum } \\
\text { entary }\end{array}$ \\
\hline $\begin{array}{l}\text { Checkma } \\
\mathrm{te}^{186}\end{array}$ & FI & $\begin{array}{l}\text { Sami } \\
\text { education } \\
\text { institute }\end{array}$ & Educational & Lada Suomenrinne & 2016 & $4 \min$ & Short \\
\hline $\begin{array}{l}\text { Christma } \\
\text { s } \\
\text { Magic }^{187}\end{array}$ & $\mathrm{NO}$ & $\begin{array}{l}\text { Medieprodu } \\
\text { ksjon } \\
\text { Høgskolen i }\end{array}$ & Educational & Inge Renate Buljo & 2013 & $6 \mathrm{~min}$ & Short \\
\hline
\end{tabular}

182 "Short Films Funded by the International Sámi Film Centre", International Sámi Film Institute, accessed May 9 2017, http://www.isfi.no/eng/films/short.

183 "CV", Liselotte Wajstedt, accessed May 9 2017, http://www.liselottewajstedt.com/bio/

184 "Calbmeravkaleams", Skábmagovat Film Festival, accessed June 22 2017, http://skabmagovat.fi/skabmagovat_2014/?page_id=8528.

185 "Skábmagovat 2002", Skábmagovat Film Festival, accessed May 9 2017, http://www.Skábmagovat.fi/Skábma_vanhat/Skábma_2002/800en.html.

186 "Checkmate", Skábmagovat Film Festival, accessed June 22 2017, http://skabmagovat.fi/skabmagovat_2014/?page_id=8147. 


\begin{tabular}{|c|c|c|c|c|c|c|c|}
\hline & & Finnmark & & & & & \\
\hline $\begin{array}{l}\text { Colors of } \\
\text { Life }^{188}\end{array}$ & $\mathrm{NO}$ & & Unknown & Harry Johansen & 2014 & $\begin{array}{l}40 \\
\min \end{array}$ & Short \\
\hline Davas $^{189}$ & SE & & Unknown & Akkil & 2016 & $3 \min$ & Short \\
\hline $\begin{array}{l}\text { Deadline } \\
\text { Torp }^{190}\end{array}$ & $\mathrm{NO}$ & $\begin{array}{l}\text { NRK } \\
\text { Drama }\end{array}$ & State media & Nils Gaup & 2005 & $\begin{array}{l}1 \mathrm{~h} 56 \\
\min \end{array}$ & Feature \\
\hline $\begin{array}{l}\text { Det } \\
\text { kommer } \\
\text { aldrig att } \\
\text { gå over }{ }^{191}\end{array}$ & SE & $\begin{array}{l}\text { Direktörn } \\
\text { och } \\
\text { Fabrikörn } \\
\text { Handelsbola } \\
\text { g }\end{array}$ & $\begin{array}{l}\text { Private } \\
\text { company }\end{array}$ & Amanda Kernell & 2011 & $\begin{array}{l}18 \\
\min \end{array}$ & Short \\
\hline $\begin{array}{l}\text { Detention } \\
192\end{array}$ & $\mathrm{CA}$ & NFB & State media & $\begin{array}{l}\text { Elle-Maija } \\
\text { Tailfeathers }\end{array}$ & 2017 & $39 \mathrm{sec}$ & Short \\
\hline $\begin{array}{l}\text { Dolastall } \\
\text { at }^{193}\end{array}$ & FI & & Unknown & Marja Helander & 2016 & $6 \mathrm{~min}$ & $\begin{array}{l}\text { Experi } \\
\text { mental }\end{array}$ \\
\hline $\begin{array}{l}\text { Du } \\
\text { lahka }\end{array}$ & & & Unknown & $\begin{array}{l}\text { Ann Mari } \\
\text { Andersen }\end{array}$ & & & \\
\hline
\end{tabular}

187 "Skábmagovat 2014", Skábmagovat Film Festival, Microsoft Word document courtesy of Kaija Anttonen.

188 "Dellie Maa Program", Dellie Maa Festival, 2014, digital PDF file courtesy of Oskar Östergren.

189 "Davas", Skábmagovat Film Festival, accessed June 22 2017, http://skabmagovat.fi/skabmagovat_2014/?page_id=8534.

190 "Deadline Torp", Salomonsson Agency, accessed June 22 2017, http://www.salomonssonagency.se/scripts/deadline-torp.

191 "Amanda Kernell", Swedish Film Institute Database, accessed May 9 2017, http://www.sfi.se/en-GB/Swedishfilm-

database/Item/?type=PERSON\&itemid=325817\&iv=MOVIE\&ref=\%2ftemplates\%2fSwedishFilmSearchResult.asp $\mathrm{x} \% 3 \mathrm{fid} \% 3 \mathrm{~d} 1225 \% 26$ epslanguage $\% 3 \mathrm{den}-$

GB\%26searchword\%3damanda+kernell\%26type \%3dPerson\%26match\%3dBegin\%26page\%3d1\%26prom\%3dFalse.

192 "Film and Video Works", Elle-Maija Tailfeathers.com, accessed May 9 2017, http://elle-maijatailfeathers.com/?page_id=311.

193 "Dolastallat", ImagineNative, accessed May 9 2017, http://www.imaginenative.org/dollastallat. 


\begin{tabular}{|c|c|c|c|c|c|c|c|}
\hline $\begin{array}{l}\text { Eahparas } \\
195\end{array}$ & NO & & Unknown & $\begin{array}{l}\text { Anne Merete } \\
\text { Gaup }\end{array}$ & 2011 & $7 \mathrm{~min}$ & Short \\
\hline $\begin{array}{l}\text { Edith and } \\
\text { Aljosja }^{196}\end{array}$ & $\mathrm{SE}$ & Julev Film & $\begin{array}{l}\text { Private } \\
\text { company }\end{array}$ & Ann Holmgren & 2015 & $8 \min$ & Short \\
\hline $\begin{array}{l}\text { Ellos } \\
\text { Sápmi }{ }^{197}\end{array}$ & SE & Julev Film & $\begin{array}{l}\text { Private } \\
\text { company }\end{array}$ & $\begin{array}{l}\text { Per-Josef } \\
\text { Idivuoma }\end{array}$ & 2015 & $\begin{array}{l}12 \\
\min \end{array}$ & Short \\
\hline $\begin{array}{l}\text { Elsa } \\
\text { Laula } \\
\text { Renberg } \\
198\end{array}$ & NO & NRK Sapmi & State media & $\begin{array}{l}\text { Per Christian } \\
\text { Olsen }\end{array}$ & 2017 & $\begin{array}{l}28 \\
\min \end{array}$ & $\begin{array}{l}\text { Docum } \\
\text { entary }\end{array}$ \\
\hline $\begin{array}{l}\text { Fievrrut } \\
199\end{array}$ & FI & & Unknown & Inger-Mari Aikio & 2015 & $3 \min$ & $\begin{array}{l}\text { Music } \\
\text { Video }\end{array}$ \\
\hline $\begin{array}{l}\text { Firekeepe } \\
\mathrm{rs}^{200}\end{array}$ & $\mathrm{NO}$ & & Unknown & Rosella Ragazzi & 2007 & $\begin{array}{l}57 \\
\min \end{array}$ & $\begin{array}{l}\text { Docum } \\
\text { entary }\end{array}$ \\
\hline $\begin{array}{l}\text { From } \\
\text { Kalahari }\end{array}$ & NO & & Unknown & & 2004 & $\begin{array}{l}28 \\
\min \end{array}$ & $\begin{array}{l}\text { Docum } \\
\text { entary }\end{array}$ \\
\hline
\end{tabular}

194 "Skábmagovat 2008 - Programme", Skábmagovat.fi, accessed June 202017, http://www.skabmagovat.fi/skabma_vanhat/skabma_2008/ohjelma_en/ohjelmainfo_su.htm\#21.

195 "Skábmagovat 2016: Kaamoksen Kuvia/Reflections of the Endless Night", Inari: Skábmagovat Film Festival, 2016.

196 "7 Sámi Films", International Sámi Film Institute, accessed May 9 2017, http://www.isfi.no/eng/films/7Sámistories.

197 "7 Sámi Films", International Sámi Film Institute, accessed May 9 2017, http://www.isfi.no/eng/films/7Sámistories.

198 "Elsa Laula Renberg", Skábmagovat Film Festival, accessed June 22 2017, http://skabmagovat.fi/skabmagovat_2014/?page_id=8440.

199 "Skábmagovat 2016: Kaamoksen Kuvia/Reflections of the Endless Night", Inari: Skábmagovat Film Festival, 2016.

200 "Skábmagovat 2008", Skábmagovat Film Festival, accessed May 9 2017, http://www.Skábmagovat.fi/Skábma_vanhat/Skábma_2008/ohjelma_en/ohjelmainfo_su.htm\#. 


\begin{tabular}{|c|c|c|c|c|c|c|c|}
\hline $\begin{array}{l}\text { to } \\
\text { Sápmi }{ }^{201}\end{array}$ & & & & & & & \\
\hline $\begin{array}{l}\text { From the } \\
\text { Fell to } \\
\text { the Ice } \\
\text { Globe } \\
\text { Theatre } \\
202\end{array}$ & $\mathrm{NO}$ & NRK & State media & Johs. Kalvemo & 2003 & $\begin{array}{l}30 \\
\min \end{array}$ & $\begin{array}{l}\text { Docum } \\
\text { entary }\end{array}$ \\
\hline $\begin{array}{l}\text { Gavavuo } \\
\text { hta }^{203}\end{array}$ & $\mathrm{RU}$ & & Unknown & Valentina Sovkina & 2016 & $\begin{array}{l}30 \\
\min \end{array}$ & Short \\
\hline $\begin{array}{l}\text { Gichikiiw } \\
\text { enging }^{204}\end{array}$ & CA & $\begin{array}{l}\text { Elle-Maija } \\
\text { Tailfeathers }\end{array}$ & Individual & $\begin{array}{l}\text { Elle-Maija } \\
\text { Tailfeathers }\end{array}$ & 2016 & $53 \mathrm{sec}$ & $\begin{array}{l}\text { Docum } \\
\text { entary }\end{array}$ \\
\hline $\begin{array}{l}\text { Giksasuv } \\
\text { van } \\
\text { Ealli } \\
\text { 205 }\end{array}$ & $\mathrm{NO}$ & Rein Film & $\begin{array}{l}\text { Private } \\
\text { company }\end{array}$ & Egil Pedersen & 2015 & $\begin{array}{l}15 \\
\min \end{array}$ & Short \\
\hline Giron $^{206}$ & SE & & Unknown & Liselotte Wajstedt & 2011 & $\begin{array}{l}3 \mathrm{~min} \\
56 \mathrm{sec}\end{array}$ & Short \\
\hline Glassduk & $\mathrm{NO}$ & Eira Film & Private & Nils Gaup & 2014 & 92 & Feature \\
\hline
\end{tabular}

201 "Skábmagovat 2006", Skábmagovat Film Festival, accessed May 9 2017, http://www.Skábmagovat.fi/Skábma_vanhat/Skábma_2006/800en.html.

202 "Skábmagovat 2004", Skábmagovat Film Festival, accessed June 202017, http://www.skabmagovat.fi/skabma_vanhat/skabma_2004/800en.html.

203 "Gavavuohta", Skábmagovat Film Festival, accessed June 222017, http://skabmagovat.fi/skabmagovat_2014/?page_id=10435.

204 "Film and Video Works", Elle-Maija Tailfeathers.com, accessed May 9 2017, http://elle-maijatailfeathers.com/?page_id=311.

205 "7 Sámi Films", International Sámi Film Institute, accessed May 92017, http://www.isfi.no/eng/films/7Sámistories/.

206 "CV", Liselotte Wajstedt, accessed May 9 2017, http://www.liselottewajstedt.com/bio. 


\begin{tabular}{|c|c|c|c|c|c|c|c|}
\hline kene $^{207}$ & & AS & company & & & $\min$ & \\
\hline Goahti $^{208}$ & $\mathrm{SE}$ & & Unknown & Liselotte Wajstedt & 2016 & $\begin{array}{l}10 \\
\min \\
26 \\
\text { secon } \\
\text { ds }\end{array}$ & $\begin{array}{l}\text { Docum } \\
\text { entary }\end{array}$ \\
\hline $\begin{array}{l}\text { Great } \\
\text { Grandmo } \\
\text { thers }^{209}\end{array}$ & $\mathrm{SE}$ & & Unknown & John Erling Utsi & & $\begin{array}{l}58 \\
\min \end{array}$ & $\begin{array}{l}\text { Docum } \\
\text { entary }\end{array}$ \\
\hline $\begin{array}{l}\text { Greater } \\
\text { than } \\
\text { ourselves } \\
210\end{array}$ & $\mathrm{NO}$ & & Unknown & Anita Lervoll & 2003 & $\begin{array}{l}35 \\
\min \end{array}$ & $\begin{array}{l}\text { Docum } \\
\text { entary }\end{array}$ \\
\hline Guolli $^{211}$ & FI & & Unknown & Jouni West & 2016 & $4 \min$ & Short \\
\hline $\begin{array}{l}\text { Guovssah } \\
\text { as oaidna } \\
\mathrm{du}^{212}\end{array}$ & & $\begin{array}{l}\text { Freedom } \\
\text { From Fear }\end{array}$ & $\begin{array}{l}\text { Private } \\
\text { company }\end{array}$ & $\begin{array}{l}\text { Sara Margrethe } \\
\text { Oskal }\end{array}$ & 2015 & $\begin{array}{l}12 \\
\min \end{array}$ & Short \\
\hline Guovza & FI & & Unknown & Paul-Anders & 1994 & & \\
\hline
\end{tabular}

207 "Glassdukkene", Norwegian Film Institute, accessed June 22 2017, http://www.nfi.no/english/123037/glassdukkene.

208 "CV", Liselotte Wajstedt, accessed May 9 2017, http://www.liselottewajstedt.com/bio.

209 "Dellie Maa Program", Dellie Maa Festival, 2013, digital PDF file courtest of Oskar Östergren.

210 "Skábmagovat 2004-05", Skábmagovat Fim Festival, accessed May 92017, http://www.Skábmagovat.fi/Skábma_vanhat/Skábma_2005/800en.html.

211 "Guolli", Skábmagovat Film Festival, accessed June 22 2017, http://skabmagovat.fi/skabmagovat_2014/?page_id=8438.

212 "7 Sámi Films", International Sámi Film Institute, accessed May 92017, http://www.isfi.no/eng/films/7Sámistories. 


\begin{tabular}{|c|c|c|c|c|c|c|c|}
\hline 213 & & & & Simma & & & \\
\hline $\begin{array}{l}\text { Hate } \\
\text { Slash } \\
\text { Love }^{214}\end{array}$ & FI & & Unknown & Elmeri Harkonen & 2015 & $\begin{array}{l}15 \\
\min \end{array}$ & \\
\hline $\begin{array}{l}\text { Hear the } \\
\text { Spirits } \\
\mathrm{Cry}^{215}\end{array}$ & $\mathrm{NO}$ & $\begin{array}{l}\text { Govas Film } \\
\& \\
\text { Mediaprodu } \\
\text { ksjon AS }\end{array}$ & $\begin{array}{l}\text { Private } \\
\text { company }\end{array}$ & Harry Johansen & 2014 & $4 \mathrm{~min}$ & $\begin{array}{l}\text { Music } \\
\text { Video }\end{array}$ \\
\hline $\begin{array}{l}\text { Heijasta } \\
\text { mattomin } \\
\text { Askelin } \\
216\end{array}$ & FI & & Unknown & Jonne Jarvinen & 2015 & $7 \mathrm{~min}$ & \\
\hline $\begin{array}{l}\text { Hej Da } \\
\text { Halleluja } \\
\mathrm{h}^{217}\end{array}$ & & & Unknown & Ann Holmgren & 2015 & $\begin{array}{l}72 \\
\min \end{array}$ & \\
\hline $\begin{array}{l}\text { Hilbes } \\
\text { Biiga }^{218}\end{array}$ & $\mathrm{NO}$ & $\begin{array}{l}\text { Marja Bal } \\
\text { Nango ja } \\
\text { Marie }\end{array}$ & Individuals & Marja Bål Nango & 2015 & $\begin{array}{l}18 \\
\min \end{array}$ & Short \\
\hline
\end{tabular}

213 "Guovza", Internet Movie Database, accessed May 92017, http://www.imdb.com/title/tt0373879/?ref_=fn_al_tt_1.

214 "Skábmagovat 2016: Kaamoksen Kuvia/Reflections of the Endless Night", Inari: Skábmagovat Film Festival, 2016.

215 "Skábmagovat 2015", Skábmagovat Film Festival, Microsoft Word document courtesy of Kaija Anttonen.

216 "Skábmagovat 2016: Kaamoksen Kuvia/Reflections of the Endless Night", Inari: Skábmagovat Film Festival, 2016.

217 "Dellie Maa Program", Dellie Maa Festival, 2016, digital PDF file courtesy of Oskar Östergren.

218 "7 Sámi Films", International Sámi Film Institute, accessed May 92017, http://www.isfi.no/eng/films/7Sámistories. 


\begin{tabular}{|c|c|c|c|c|c|c|c|}
\hline & & Lagerkvist & & & & & \\
\hline Hilda $^{219}$ & FI & $\begin{array}{l}\text { Sami } \\
\text { education } \\
\text { institute }\end{array}$ & Educational & Niki Rasmus & 2016 & $7 \min$ & Short \\
\hline $\begin{array}{l}\text { Hjerterått } \\
220\end{array}$ & $\mathrm{NO}$ & NRK & State media & Nils Gaup & 2013 & $\begin{array}{l}8 \times 25 \\
\min \end{array}$ & $\begin{array}{l}\text { TV } \\
\text { series }\end{array}$ \\
\hline $\begin{array}{l}\text { How Far } \\
\text { Will You } \\
\text { Go to } \\
\text { Vote? }\end{array}$ & $\mathrm{CA}$ & $\begin{array}{l}\text { Elle-Maija } \\
\text { Tailfeathers }\end{array}$ & Individual & $\begin{array}{l}\text { Elle-Maija } \\
\text { Tailfeathers }\end{array}$ & 2015 & $1 \mathrm{~min}$ & Short \\
\hline $\begin{array}{l}\text { Hur gor } \\
\text { man for } \\
\text { att rada } \\
\text { ett folk }\end{array}$ & SE & $\begin{array}{l}\text { Världsålder } \\
\mathrm{AB} \text { in } \\
\text { cooperation } \\
\text { with } \\
\text { Sveriges } \\
\text { Television } \\
\text { and } \\
\text { Filmpool } \\
\text { Nord }\end{array}$ & $\begin{array}{l}\text { Mixed } \\
\text { (private } \\
\text { company, } \\
\text { state media) }\end{array}$ & Maja Hagerman & 2014 & $\begin{array}{l}58 \\
\min \end{array}$ & $\begin{array}{l}\text { Docum } \\
\text { entary }\end{array}$ \\
\hline Hurry Up & CA & Elle-Maija & Individual & Elle-Maija & 2015 & 10 & Docum \\
\hline
\end{tabular}

219 "Hilda", Skábmagovat Film Festival, accessed June 22 2017, http://skabmagovat.fi/skabmagovat_2014/?page_id=8322.

220 "Hjerterått", NRK, accessed June 22 2017, http://tv.nrksuper.no/serie/hjerteraatt.

221 "Film and Video Works", Elle-Maija Tailfeathers.com, accessed May 9 2017, http://elle-maijatailfeathers.com/?page_id=311.

222 "Dellie Maa Program", Dellie Maa Festival, 2014, digital PDF file courtesy of Oskar Östergren. 


\begin{tabular}{|c|c|c|c|c|c|c|c|}
\hline $\begin{array}{l}\text { You } \\
\text { Stupid } \\
\text { Cripple }^{223}\end{array}$ & & Tailfeathers & & $\begin{array}{l}\text { Tailfeathers, } \\
\text { Terreane Derrick }\end{array}$ & & $\min$ & entary \\
\hline $\begin{array}{l}\text { I am in } \\
\text { Lavvu }^{224}\end{array}$ & SE & & Unknown & Liselotte Wajstedt & 2011 & $\begin{array}{l}4 \mathrm{~min} \\
56 \mathrm{sec}\end{array}$ & Short \\
\hline $\begin{array}{l}\text { I Have a } \\
\text { Friend }^{225}\end{array}$ & FI & $\begin{array}{l}\text { Sámi } \\
\text { educational } \\
\text { centre }\end{array}$ & Educational & $\begin{array}{l}\text { Ritva Torika- } \\
\text { Gelencser }\end{array}$ & 2004 & $6 \mathrm{~min}$ & $\begin{array}{l}\text { Docum } \\
\text { entary }\end{array}$ \\
\hline $\begin{array}{l}\text { I Will } \\
\text { Always } \\
\text { Love You } \\
\text { Kingen } 226\end{array}$ & $\mathrm{SE}$ & $\begin{array}{l}\text { Bob Film } \\
\text { Sweden AB }\end{array}$ & $\begin{array}{l}\text { Private } \\
\text { company }\end{array}$ & Amanda Kernell & 2017 & & Short \\
\hline $\begin{array}{l}\text { Icebreake } \\
\text { r }\end{array}$ & NO & & Unknown & Elle Marja Eira & 2016 & $3 \mathrm{~min}$ & $\begin{array}{l}\text { Music } \\
\text { Video }\end{array}$ \\
\hline $\begin{array}{l}\text { Iditsilba } \\
227\end{array}$ & & Davas Film & $\begin{array}{l}\text { Private } \\
\text { company }\end{array}$ & Elle Marja Eira & 2015 & $\begin{array}{l}12 \\
\min \end{array}$ & Short \\
\hline Ima & FI & & Unknown & Ima Aikio & 2015 & & Music \\
\hline
\end{tabular}

223 "Film and Video Works", Elle-Maija Tailfeathers.com, accessed May 9 2017, http://elle-maijatailfeathers.com/?page_id=311.

224 "CV", Liselotte Wajstedt, accessed May 9 2017, http://www.liselottewajstedt.com/bio.

225 "7 Sámi Films", International Sámi Film Institute, accessed May 92017, http://www.isfi.no/eng/films/7Sámistories.

226 "Amanda Kernell", Swedish Film Institute Database, accessed May 9 2017, http://www.sfi.se/en-GB/Swedishfilm-

database $/$ Item/?type=PERSON\&itemid=325817\&iv=MOVIE\&ref=\%2ftemplates $\% 2 \mathrm{fS}$ wedishFilmSearchResult.asp $\mathrm{x} \% 3 \mathrm{fid} \% 3 \mathrm{~d} 1225 \% 26$ epslanguage $\% 3 \mathrm{den}-$

GB\%26searchword\%3damanda+kernell\%26type\%3dPerson\%26match\%3dBegin\%26page\%3d1\%26prom\%3dFalse.

227 "Short Films Funded by the International Sámi Film Centre", International Sámi Film Institute, accessed May 9 2017, http://www.isfi.no/eng/films/short. 


\begin{tabular}{|c|c|c|c|c|c|c|c|}
\hline $\begin{array}{l}\text { Hutkosat } \\
-12 \\
\text { Musihkka } \\
\text { video }^{228}\end{array}$ & & & & & & & Video \\
\hline $\begin{array}{l}\text { In the } \\
\text { Stream of } \\
\text { Life }^{229}\end{array}$ & NO & & Unknown & Anstein Mikkelsen & 2003 & $\begin{array}{l}50 \\
\min \end{array}$ & Short \\
\hline $\begin{array}{l}\text { Ivnnit ja } \\
\text { eallin }{ }^{230}\end{array}$ & & $\begin{array}{l}\text { Harry } \\
\text { Johansen, } \\
\text { Govas }\end{array}$ & $\begin{array}{l}\text { Mixed } \\
\text { (individual, } \\
\text { private } \\
\text { company) }\end{array}$ & Harry Johansen & & $\begin{array}{l}40 \\
\min \end{array}$ & $\begin{array}{l}\text { Docum } \\
\text { entary }\end{array}$ \\
\hline $\begin{array}{l}\text { Jahki il } \\
\text { leat jagi } \\
\text { viellja }^{231}\end{array}$ & $\mathrm{NO}$ & & Unknown & Mai-Lis Eira & 2015 & $\begin{array}{l}12 \\
\min \end{array}$ & Short \\
\hline $\begin{array}{l}\text { Job } \\
\text { Interview } \\
232\end{array}$ & FI & & Unknown & & 2006 & $5 \mathrm{~min}$ & \\
\hline Joik $^{233}$ & SE & & Unknown & Maj-Lis Skaltje & 2014 & 85 & Docum \\
\hline
\end{tabular}

228 "Skábmagovat 2016: Kaamoksen Kuvia/Reflections of the Endless Night", Inari: Skábmagovat Film Festival, 2016.

229 "Skábmagovat 2004", Skábmagovat Film Festival, accessed May 9 2017, http://www.Skábmagovat.fi/Skábma_vanhat/Skábma_2004/800en.html.

230 "Documentary Films Funded by the International Sámi Film Centre", International Sámi Film Institute, accessed May 9 2017, http://www.isfi.no/eng/films/documentary.

231 "Skábmagovat 2016: Kaamoksen Kuvia/Reflections of the Endless Night", Inari: Skábmagovat Film Festival, 2016.

232 "Skábmagovat 2006", Skábmagovat Film Festival, accessed May 9 2017, http://www.Skábmagovat.fi/Skábma_vanhat/Skábma_2006/800en.html.

233 "Dellie Maa Program", Dellie Maa Festival, 2015, digital PDF file courtesy of Oskar Östergren. 


\begin{tabular}{|c|c|c|c|c|c|c|c|}
\hline & & & & & & $\min$ & entary \\
\hline $\begin{array}{l}\text { Jorinda/C } \\
\text { herry on } \\
\text { Top }^{234}\end{array}$ & $\mathrm{SE}$ & & Unknown & Liselotte Wajstedt & 2014 & $9 \min$ & Short \\
\hline $\begin{array}{l}\text { Jorindas } \\
\text { Resa }^{235}\end{array}$ & SE & & Unknown & Liselotte Wajstedt & 2014 & $\begin{array}{l}16 \\
\min \end{array}$ & Short \\
\hline $\begin{array}{l}\text { Juoigangi } \\
\text { ehta }^{236}\end{array}$ & & $\begin{array}{l}\text { Kautokeino } \\
\text { Film }\end{array}$ & $\begin{array}{l}\text { Private } \\
\text { company }\end{array}$ & $\begin{array}{l}\text { Elle Sofe } \\
\text { Henriksen }\end{array}$ & 2011 & $5 \mathrm{~min}$ & Short \\
\hline $\begin{array}{l}\text { Just Do } \\
\mathrm{It}^{237}\end{array}$ & $\mathrm{NO}$ & $\begin{array}{l}\text { Norsk } \\
\text { Filminstitut } \\
\text { et, Eva } \\
\text { Faerevagg }\end{array}$ & $\begin{array}{l}\text { Mixed } \\
\text { (individual, } \\
\text { government } \\
\text { organization) }\end{array}$ & Nils Gaup & 1994 & & Short \\
\hline $\begin{array}{l}\text { Juuret } \\
\text { on }^{238}\end{array}$ & FI & & $\begin{array}{l}\text { Mixed } \\
\text { (individual, } \\
\text { private } \\
\text { company) }\end{array}$ & $\begin{array}{l}\text { Suvi West, Anssi } \\
\text { Kömi }\end{array}$ & 2016 & $\begin{array}{l}13 \\
\min \end{array}$ & Short \\
\hline $\begin{array}{l}\text { Kaisa's } \\
\text { Enchante }\end{array}$ & & $\begin{array}{l}\text { Satu } \\
\text { Maijava, }\end{array}$ & & Katja Gauriloff & & & $\begin{array}{l}\text { Docum } \\
\text { entary }\end{array}$ \\
\hline
\end{tabular}

234 "CV", Liselotte Wajstedt, accessed May 9 2017, http://www.liselottewajstedt.com/bio.

235 "CV", Liselotte Wajstedt, accessed May 9 2017, http://www.liselottewajstedt.com/bio.

236 "Short Films Funded by the International Sámi Film Centre", International Sámi Film Institute, accessed May 9 2017, http://www.isfi.no/eng/films/short.

237 "Just Do It", filmrommet.no, accessed June 22 2017, https://www.filmrommet.no/film/details.aspx?filmid=44880.

238 "Juuret On", Skábmagovat Film Festival, accessed June 22 2017, http://skabmagovat.fi/skabmagovat_2014/?page_id=8430. 


\begin{tabular}{|c|c|c|c|c|c|c|c|}
\hline $\begin{array}{l}\mathrm{d} \\
\text { Forest }^{239}\end{array}$ & & $\begin{array}{l}\text { Oktober } \\
\text { Film }\end{array}$ & & & & & \\
\hline $\begin{array}{l}\text { Kaissi- } \\
\text { U'lljan- } \\
\text { Mä’rjj- } \\
\text { U'lljan - } \\
\text { Heidi } \\
\text { Gauriloff } \\
240\end{array}$ & FI & $\begin{array}{l}\text { Sami } \\
\text { education } \\
\text { institute }\end{array}$ & Educational & Aleksi Ahlakorpi & 2016 & $\begin{array}{l}10 \\
\min \end{array}$ & $\begin{array}{l}\text { Docum } \\
\text { entary }\end{array}$ \\
\hline $\begin{array}{l}\text { Kielen } \\
\text { Päällä }\end{array}$ & FI & $\begin{array}{l}\text { Sami } \\
\text { education } \\
\text { institute }\end{array}$ & Educational & $\begin{array}{l}\text { Piia Kangas, Lada } \\
\text { Suomenrinne }\end{array}$ & 2016 & $5 \min$ & Short \\
\hline $\begin{array}{l}\text { Kiruna } \\
\text { Rymdväg } \\
\text { en }^{241}\end{array}$ & SE & $\begin{array}{l}\text { Filmpool } \\
\text { Nord, Sirel } \\
\text { Peensaar } \\
\text { och } \\
\text { Sveriges } \\
\text { Telivision } \\
\text { AB, Eva } \\
\text { Sandelin, }\end{array}$ & $\begin{array}{l}\text { Mixed } \\
\text { (private } \\
\text { company, } \\
\text { government } \\
\text { organization, } \\
\text { individual) }\end{array}$ & Liselotte Wajstedt & 2013 & $\begin{array}{l}52 \\
\min \end{array}$ & $\begin{array}{l}\text { Docum } \\
\text { entary }\end{array}$ \\
\hline
\end{tabular}

239 "Documentary Films Funded by the International Sámi Film Centre", International Sámi Film Institute, accessed May 9 2017, http://www.isfi.no/eng/films/documentary.

240 "Kaissi-U'lljan-Mä’rjj-U'lljan - Heidi Gauriloff", Skábmagovat Film Festival, accessed June 22 2017, http://skabmagovat.fi/skabmagovat_2014/?page_id=8335.

241 "CV", Liselotte Wajstedt, accessed May 9 2017, http://www.liselottewajstedt.com/bio. 


\begin{tabular}{|c|c|c|c|c|c|c|c|}
\hline & & $\begin{array}{l}\text { LittleBig } \\
\text { Productions } \\
\text {, SFI }\end{array}$ & & & & & \\
\hline $\begin{array}{l}\text { Kiruna } \\
\text { the } \\
\text { Driftbloc } \\
\mathrm{k}^{242}\end{array}$ & SE & & Unknown & Liselotte Wajstedt & $\begin{array}{l}2016 \\
\text { (ong } \\
\text { oing }\end{array}$ & & $\begin{array}{l}\text { Experi } \\
\text { mental }\end{array}$ \\
\hline $\begin{array}{l}\text { Leaving } \\
\text { the } \\
\text { Herd }^{243}\end{array}$ & $\mathrm{NO}$ & $\begin{array}{l}\text { Mathis } \\
\text { Ståle } \\
\text { Mathisen, } \\
\text { Rein Film }\end{array}$ & $\begin{array}{l}\text { Mixed } \\
\text { (individual, } \\
\text { private } \\
\text { company) }\end{array}$ & Egil Pedersen & 2013 & $5 \min$ & Short \\
\hline $\begin{array}{l}\text { Leivänm } \\
\text { uruseni }^{244}\end{array}$ & FI & & Unknown & Oskari Sipola & 2011 & $3 \mathrm{~min}$ & Short \\
\hline $\operatorname{Lies}^{245}$ & FI & & Unknown & $\begin{array}{l}\text { Ville-Riiko } \\
\text { Fofonoff }\end{array}$ & 2016 & $5 \mathrm{~min}$ & $\begin{array}{l}\text { Music } \\
\text { Video }\end{array}$ \\
\hline $\begin{array}{l}\text { Ludiin } \\
\text { Nahkkui } \\
246\end{array}$ & NO & & Unknown & Johan Sara Jr. & 2014 & $3 \min$ & Short \\
\hline Luhtat's & FI & Sámi & Educational & Ritva Torika- & 2004 & 10 & Short \\
\hline
\end{tabular}

242 "CV", Liselotte Wajstedt, accessed May 9 2017, http://www.liselottewajstedt.com/bio.

243 "Skábmagovat 2015", Skábmagovat Film Festival, Microsoft Word document courtesy of Kaija Anttonen.

244 "Skábmagovat 2012", Skábmagovat Film Festival, accessed May 9 2017, http://www.Skábmagovat.fi/Skábma_vanhat/Skábma_2012/800en.html.

245 "Lies", Skábmagovat Film Festival, accessed June 222017,

http://skabmagovat.fi/skabmagovat_2014/?page_id=8644.

246 "Ludiin Nahkkui", Skábmagovat Film Festival, accessed June 22 2017,

http://skabmagovat.fi/skabmagovat_2014/?page_id=8057. 


\begin{tabular}{|c|c|c|c|c|c|c|c|}
\hline Lunch $^{247}$ & & $\begin{array}{l}\text { educational } \\
\text { centre }\end{array}$ & & Gelencser & & $\min$ & \\
\hline $\begin{array}{l}\text { Maan } \\
\text { muisti }{ }^{248}\end{array}$ & FI & $\begin{array}{l}\text { Giron Filmi } \\
\text { Oy }\end{array}$ & $\begin{array}{l}\text { Private } \\
\text { company }\end{array}$ & $\begin{array}{l}\text { Markku } \\
\text { Lehmuskallio, } \\
\text { Anastasia Lapsui }\end{array}$ & 2009 & & $\begin{array}{l}\text { Docum } \\
\text { entary }\end{array}$ \\
\hline $\begin{array}{l}\text { Maccâm } \\
\text { madduid } \\
- \\
\text { tánssájeij } \\
\text { ee } \\
\text { maainâs }\end{array}$ & FI & $\begin{array}{l}\text { Sami } \\
\text { education } \\
\text { institute }\end{array}$ & Educational & $\begin{array}{l}\text { Maria-Riitta } \\
\text { Mällinen }\end{array}$ & 2016 & $\begin{array}{l}10 \\
\min \end{array}$ & $\begin{array}{l}\text { Docum } \\
\text { entary }\end{array}$ \\
\hline Maison $^{249}$ & SE & & Unknown & Ann Holmgren & 2005 & $7 \mathrm{~min}$ & \\
\hline $\begin{array}{l}\text { Majja ja } \\
\text { Sujujujja } \\
250\end{array}$ & $\mathrm{NO}$ & NRK & State media & Sverre Porsanger & 2004 & $\begin{array}{l}90 \\
\min \end{array}$ & Feature \\
\hline $\begin{array}{l}\text { Märät } \\
\text { säppikkää } \\
\mathrm{t}^{251}\end{array}$ & FI & $\begin{array}{l}\text { Yleisradio } \\
\text { TV } 2\end{array}$ & State media & $\begin{array}{l}\text { Anne Kirste } \\
\text { Aikio/Suvi West }\end{array}$ & 2009 & & $\begin{array}{l}\mathrm{TV} \\
\text { product } \\
\text { ion }\end{array}$ \\
\hline
\end{tabular}

247 "Skábmagovat 2004-05", Skábmagovat Film Festival, accessed May 9 2017, http://www.Skábmagovat.fi/Skábma_vanhat/Skábma_2005/800en.html.

${ }^{248}$ Skábmagovat 2010, Skábmagovat Film Festival, accessed May 92017 , http://www.Skábmagovat.fi/Skábma_vanhat/Skábma_2010/800en.html.

249 "Skábmagovat 2006", Skábmagovat Film Festival, accessed May 9 2017, http://www.Skábmagovat.fi/Skábma_vanhat/Skábma_2006/800en.html.

${ }^{250}$ Skábmagovat 2004-05", Skábmagovat Film Festival, accessed May 9 2017, http://www.Skábmagovat.fi/Skábma_vanhat/Skábma_2005/800en.html.

251 "Skábmagovat 2010", Skábmagovat Film Festival, accessed May 9 2017, http://www.Skábmagovat.fi/Skábma_vanhat/Skábma_2010/800en.html. 


\begin{tabular}{|l|l|l|l|l|l|l|l|}
\hline Maverick & CA & & Unknown & Elle-Maija & 2015 & 11 & Docum \\
ssther & & & Tailfeathers & & min \\
Tailfeath \\
ers, \\
Blood \\
Reserve \\
252
\end{tabular}

252 "Film and Video Works", Elle-Maija Tailfeathers.com, accessed May 9 2017, http://elle-maijatailfeathers.com/?page_id=311.

253 "Skábmagovat 2016: Kaamoksen Kuvia/Reflections of the Endless Night", Inari: Skábmagovat Film Festival, 2016.

254 "Skábmagovat 2015", Skábmagovat Film Festival, Microsoft Word document courtesy of Kaija Anttonen.

255 "Skábmagovat 2016: Kaamoksen Kuvia/Reflections of the Endless Night", Inari: Skábmagovat Film Festival, 2016.

256 "Skábmagovat 2016: Kaamoksen Kuvia/Reflections of the Endless Night", Inari: Skábmagovat Film Festival, 2016. 


\begin{tabular}{|l|l|l|l|l|l|l|l|}
\hline $\begin{array}{l}\text { Min } \\
\text { Mormor } \\
\text { och jag }^{257}\end{array}$ & SE & & Unknown & Ann-Christine & 2006 & 13 & Docum \\
\hline Mino $^{258}$ & NO & & Haupt & & & \\
entary
\end{tabular}

257" Skábmagovat 2008", Skábmagovat Film Festival, accessed May 17 2017,

http://www.Skábmagovat.fi/Skábma_vanhat/Skábma_2008/ohjelma_en/ohjelmainfo_su.htm\#.

258 "Mino", Skábmagovat Film Festival, accessed May 22 2017,

http://skabmagovat.fi/skabmagovat_2014/?page_id=8672.

259 "Skábmagovat 2015", Skábmagovat Film Festival, Microsoft Word document courtesy of Kaija Anttonen.

260 "My Girlfriend's Grandma", Skábmagovat Film Festival, accessed June 22 2017,

http://skabmagovat.fi/skabmagovat_2014/?page_id=8326.

261 "Skábmagovat 2015", Skábmagovat Film Festival, Microsoft Word document courtesy of Kaija Anttonen.

262 "Skábmagovat 2014", Skábmagovat Film Festival, Microsoft Word document courtesy of Kaija Anttonen. 


\begin{tabular}{|c|c|c|c|c|c|c|c|}
\hline $\mathrm{Nini}^{263}$ & NO & NRK & State media & Nils Gaup & 1998 & $\begin{array}{l}5 \times 55 \\
\min \end{array}$ & $\begin{array}{l}\mathrm{TV} \\
\text { series }\end{array}$ \\
\hline $\begin{array}{l}\text { North } \\
\text { Star }^{264}\end{array}$ & $\begin{array}{l}\text { NO/FR/ } \\
\text { UK/IT }\end{array}$ & $\begin{array}{l}\text { Warner } \\
\text { Bros., } \\
\text { AFCL } \\
\text { Prods., M6 } \\
\text { and Federal } \\
\text { Films co- } \\
\text { production }\end{array}$ & $\begin{array}{l}\text { Private } \\
\text { company }\end{array}$ & Nils Gaup & 1996 & $\begin{array}{l}1 \mathrm{~h} 30 \\
\min \end{array}$ & Feature \\
\hline $\begin{array}{l}\text { Oktavuoh } \\
\text { ta }^{265}\end{array}$ & FI & $\begin{array}{l}\text { Sami } \\
\text { education } \\
\text { institute }\end{array}$ & Educational & $\begin{array}{l}\text { Aleksi Ahlakorpi, } \\
\text { Tomi Rantanen }\end{array}$ & 2016 & $4 \min$ & Short \\
\hline $\operatorname{Olga}^{266}$ & $\begin{array}{l}\text { SE/NO/ } \\
\mathrm{FI} / \mathrm{RU}\end{array}$ & Safi Oy & $\begin{array}{l}\text { Private } \\
\text { company }\end{array}$ & $\begin{array}{l}\text { Paul-Anders } \\
\text { Simma }\end{array}$ & 2013 & $\begin{array}{l}67 \\
\min \end{array}$ & $\begin{array}{l}\text { Docum } \\
\text { entary }\end{array}$ \\
\hline $\begin{array}{l}\text { Our } \\
\text { Songs }{ }^{267}\end{array}$ & NO & $\begin{array}{l}\text { NRK Sápmi } \\
\text { / World } \\
\text { Indigenous } \\
\text { Television }\end{array}$ & $\begin{array}{l}\text { Mixed (state } \\
\text { media, non- } \\
\text { profit) }\end{array}$ & $\begin{array}{l}\text { Ole Mahtte H. } \\
\text { Gaup }\end{array}$ & 2014 & $\begin{array}{l}3 \times 29 \\
\min \end{array}$ & $\begin{array}{l}\text { TV } \\
\text { Product } \\
\text { ion }\end{array}$ \\
\hline
\end{tabular}

${ }^{263}$ Skábmagovat 2014", Skábmagovat Film Festival, Microsoft Word document courtesy of Kaija Anttonen.

${ }^{264}$ Joe Leydon, "Review - North Star French-British-Norwegian Italian", Variety, November 4 1996, http://variety.com/1996/film/reviews/north-star-french-british-norwegian-italian-1200447837.

265 "Oktavuohta", Skábmagovat Film Festival,

${ }^{266}$ Olga, Internet Movie Database, accessed May 9 2017, http://www.imdb.com/title/tt2646256/?ref_=nm_flmg_dr_1.

267 "Skábmagovat 2015", Skábmagovat Film Festival, Microsoft Word document courtesy of Kaija Anttonen. 


\begin{tabular}{|c|c|c|c|c|c|c|c|}
\hline & & $\begin{array}{l}\text { Broadcaster } \\
\text { s Network } \\
\text { (WITBN) }\end{array}$ & & & & & \\
\hline $\begin{array}{l}\text { Paradiset } \\
268\end{array}$ & SE & $\begin{array}{l}\text { Salmonfox } \\
\text { AB }\end{array}$ & $\begin{array}{l}\text { Private } \\
\text { Company }\end{array}$ & Amanda Kernell & 2014 & $\begin{array}{l}20 \\
\min \end{array}$ & Short \\
\hline $\begin{array}{l}\text { Porot } \\
\text { Kuuluvat } \\
\text { Tuulelle } \\
269\end{array}$ & FI & & Unknown & $\begin{array}{l}\text { Paivi Kapiainen- } \\
\text { Heiskanen }\end{array}$ & 2015 & $\begin{array}{l}39 \\
\min \end{array}$ & $\begin{array}{l}\text { Docum } \\
\text { entary }\end{array}$ \\
\hline $\begin{array}{l}\text { Portraits } \\
\text { of } \\
\text { Varanger } \\
270\end{array}$ & $\mathrm{NO}$ & & Unknown & Egil Pedersen & 2013 & $\begin{array}{l}13 \\
\min \end{array}$ & $\begin{array}{l}\text { Docum } \\
\text { entary }\end{array}$ \\
\hline $\begin{array}{l}\text { Reindeer } \\
\text { Dreaming } \\
271\end{array}$ & & & Unknown & Aanti Haase & 2001 & $\begin{array}{l}27 \\
\min \end{array}$ & \\
\hline $\begin{array}{l}\text { Reindeer } \\
\text { Herding }\end{array}$ & $\mathrm{NO}$ & Siivet & $\begin{array}{l}\text { Private } \\
\text { Company }\end{array}$ & $\begin{array}{l}\text { Anstein } \\
\text { Mikkelsen, Alf }\end{array}$ & 2004 & $\begin{array}{l}25 \\
\min \end{array}$ & Short \\
\hline
\end{tabular}

268 "Amanda Kernell", Swedish Film Institute Database, accessed May 9 2017, http://www.sfi.se/en-GB/Swedishfilm-

database/Item/?type=PERSON\&itemid=325817\&iv=MOVIE\&ref=\%2ftemplates $\% 2 \mathrm{fS}$ wedishFilmSearchResult.asp $\mathrm{x} \% 3$ fid $\% 3 \mathrm{~d} 1225 \% 26$ epslanguage $\% 3 \mathrm{den}-$

GB $\% 26$ searchword\%3damanda+kernell\%26type $\% 3 \mathrm{dPerson} \% 26$ match $\% 3 \mathrm{dBegin} \% 26 \mathrm{page} \% 3 \mathrm{~d} 1 \% 26 \mathrm{prom} \% 3 \mathrm{dFalse}$. 269 "Skábmagovat 2016: Kaamoksen Kuvia/Reflections of the Endless Night", Inari: Skábmagovat Film Festival, 2016.

270 "Dellie Maa Program", Dellie Maa Festival, 2014, digital PDF file courtesy of Oskar Östergren.

271 "Skábmagovat 2002", Skábmagovat Film Festival, accessed May 9 2017, http://www.Skábmagovat.fi/Skábma_vanhat/Skábma_2002/800en.html. 


\begin{tabular}{|c|c|c|c|c|c|c|c|}
\hline $\begin{array}{l}\text { or Into } \\
\text { the } \\
\text { World }^{272}\end{array}$ & & & & Johansen & & & \\
\hline $\begin{array}{l}\text { Reindeers } \\
273\end{array}$ & SE & & Unknown & Liselotte Wajstedt & 2008 & & \\
\hline $\begin{array}{l}\text { Reisen } \\
\text { till } \\
\text { julestjern } \\
\text { en }^{274}\end{array}$ & $\mathrm{NO}$ & $\begin{array}{l}\text { Moskus } \\
\text { Film }\end{array}$ & $\begin{array}{l}\text { Private } \\
\text { Company }\end{array}$ & Nils Gaup & 2012 & $\begin{array}{l}1 \mathrm{~h} 20 \\
\min \end{array}$ & Feature \\
\hline $\begin{array}{l}\text { Sää'nte'm } \\
\text { es }^{275}\end{array}$ & FI & & Unknown & $\begin{array}{l}\text { Working group of } \\
\text { Media Programme } \\
\text { students/Erkki } \\
\text { Feodoroff }\end{array}$ & 2016 & $7 \mathrm{~min}$ & $\begin{array}{l}\text { Music } \\
\text { Video }\end{array}$ \\
\hline $\begin{array}{l}\text { Sahtan ja } \\
\text { Mahtan } \\
276\end{array}$ & FI & & Unknown & $\begin{array}{l}\text { Tarmo Lehtosalo, } \\
\text { Ailu Valle, Raquel } \\
\text { Raqn }\end{array}$ & 2012 & $4 \mathrm{~min}$ & $\begin{array}{l}\text { Music } \\
\text { Video }\end{array}$ \\
\hline $\begin{array}{l}\text { Sameblod } \\
277\end{array}$ & SE & $\begin{array}{l}\text { Nordisk } \\
\text { Filmprodukt }\end{array}$ & $\begin{array}{l}\text { Private } \\
\text { Company }\end{array}$ & Amanda Kernell & 2017 & $\begin{array}{l}\text { 1h } 50 \\
\min \end{array}$ & Feature \\
\hline
\end{tabular}

272 "Skábmagovat 2005", Skábmagovat Film Festival, accessed June 22 2017, http://www.skabmagovat.fi/skabma_vanhat/skabma_2005/800en.html.

${ }^{273}$ CV", Liselotte Wajstedt, accessed May 9 2017, http://www.liselottewajstedt.com/bio.

274 "The Journey to the Christmas Star", Norwegian Film Institute, accessed June 222017 , http://www.nfi.no/english/search/film?key=98221.

275 "Sää'nte'mes", Skábmagovat Film Festival, accessed June 222017, http://skabmagovat.fi/skabmagovat_2014/?page_id=8119.

276"Skábmagovat 2016: Kaamoksen Kuvia/Reflections of the Endless Night", Inari: Skábmagovat Film Festival, 2016.

277 "Amanda Kernell", Swedish Film Institute Database, accessed May 9 2017, http://www.sfi.se/en-GB/Swedishfilm- 


\begin{tabular}{|c|c|c|c|c|c|c|c|}
\hline & & $\begin{array}{l}\text { ion Sverige } \\
\mathrm{AB}\end{array}$ & & & & & \\
\hline $\begin{array}{l}\text { Sámi } \\
\text { Boja }^{278}\end{array}$ & $\mathrm{NO}$ & $\begin{array}{l}\text { Kautokeino } \\
\text { Film/EYEF } \\
\text { EED DOCS }\end{array}$ & $\begin{array}{l}\text { Private } \\
\text { Company }\end{array}$ & $\begin{array}{l}\text { Elle Sofe } \\
\text { Henriksen }\end{array}$ & 2015 & $9 \min$ & Short \\
\hline $\begin{array}{l}\text { Sammakk } \\
\text { o, joka oli } \\
\text { aika } \\
\text { ruma }^{279}\end{array}$ & FI & & Unknown & $\begin{array}{l}\text { Jouni West, Pia } \\
\text { Mikkila }\end{array}$ & 2010 & $4 \min$ & Short \\
\hline $\begin{array}{l}\text { Sapmi } \\
\text { Love }^{280}\end{array}$ & SE & & Unknown & $\begin{array}{l}\text { Lisa Marie } \\
\text { Kristensen }\end{array}$ & 2016 & $\begin{array}{l}28 \\
\min \end{array}$ & Short \\
\hline $\begin{array}{l}\text { Sápmi } \\
\text { Sessions } \\
281\end{array}$ & SE & SVT & State media & & 2014 & $\begin{array}{l}30 \\
\min \end{array}$ & $\begin{array}{l}\text { TV } \\
\text { Product } \\
\text { ion }\end{array}$ \\
\hline $\begin{array}{l}\text { Semester } \\
\text { systern }^{282}\end{array}$ & SE & $\begin{array}{l}\text { Direktörn } \\
\text { och }\end{array}$ & $\begin{array}{l}\text { Private } \\
\text { company }\end{array}$ & Amanda Kernell & 2008 & $\begin{array}{l}13 \\
\min \end{array}$ & Short \\
\hline
\end{tabular}

database/Item/?type=PERSON\&itemid=325817\&iv=MOVIE\&ref=\%2ftemplates\%2fSwedishFilmSearchResult.asp $\mathrm{x} \% 3$ fid $\% 3 \mathrm{~d} 1225 \% 26$ epslanguage $\% 3 \mathrm{den}-$

GB\%26searchword\%3damanda+kernell\%26type \%3dPerson\%26match\%3dBegin\%26page\%3d1\%26prom\%3dFalse. 278 "7 Sámi Films", International Sámi Film Institute, accessed May 92017, http://www.isfi.no/eng/films/7Sámistories/.

279 "Skábmagovat 2016: Kaamoksen Kuvia/Reflections of the Endless Night", Inari: Skábmagovat Film Festival, 2016.

280 "Sápmi Love", Skábmagovat Film Festival, accessed June 22 2017, http://skabmagovat.fi/skabmagovat_2014/?page_id=8681.

281 "Skábmagovat 2014", Skábmagovat Film Festival, Microsoft Word document courtesy of Kaija Anttonen.

282 "Amanda Kernell", Swedish Film Institute Database, accessed May 9 2017, http://www.sfi.se/en-GB/Swedishfilm-

database $/$ Item/?type=PERSON\&itemid=325817\&iv=MOVIE\&ref=\%2ftemplates\%2fSwedishFilmSearchResult.asp $\mathrm{x} \% 3$ fid $\% 3 \mathrm{~d} 1225 \% 26$ epslanguage $\% 3 \mathrm{den}-$

GB\%26searchword\%3damanda+kernell\%26type \%3dPerson\%26match\%3dBegin\%26page\%3d1\%26prom\%3dFalse. 


\begin{tabular}{|c|c|c|c|c|c|c|c|}
\hline & & $\begin{array}{l}\text { Fabrikörn } \\
\text { Handelsbola } \\
\text { g, } \\
\text { Dreamfield } \\
\text { Group }\end{array}$ & & & & & \\
\hline $\begin{array}{l}\text { Shipwrec } \\
\operatorname{ked}^{283}\end{array}$ & $\begin{array}{l}\mathrm{NO} / \mathrm{SE} / \\
\mathrm{USA}\end{array}$ & $\begin{array}{l}\text { Walt } \\
\text { Disney } \\
\text { Pictures, } \\
\text { Filmkamera } \\
\text { tene AS }\end{array}$ & $\begin{array}{l}\text { Private } \\
\text { company }\end{array}$ & Nils Gaup & 1990 & $\begin{array}{l}1 \mathrm{~h} 32 \\
\min \end{array}$ & Feature \\
\hline $\begin{array}{l}\text { Shitty } \\
\text { Shitty } \\
\text { Bang } \\
\text { Bang }^{284}\end{array}$ & NO & $\begin{array}{l}\text { Cecilia } \\
\text { Sundkvist }\end{array}$ & Individual & Kjartan Nilsen & 2014 & $4 \min$ & Short \\
\hline $\begin{array}{l}\text { Small } \\
\text { Fish } \\
\text { Crying }^{285}\end{array}$ & FI & $\begin{array}{l}\text { Ima filbma- } \\
\text { ja } \\
\text { sátneduodji } \\
\text { \& Inarin } \\
\text { elokuvateoll } \\
\text { isuus }\end{array}$ & $\begin{array}{l}\text { Mixed } \\
\text { (private } \\
\text { company, } \\
\text { non-profit) }\end{array}$ & $\begin{array}{l}\text { Ima Aikio- } \\
\text { Arianaick }\end{array}$ & 2014 & $3 \min$ & $\begin{array}{l}\text { Music } \\
\text { Video }\end{array}$ \\
\hline
\end{tabular}

${ }^{283}$ Mark Caro, "Shipwrecked Springs Leaks But Doesn't Sink", Chicago Tribune, March 3 1991, accessed June 22 2017, http://articles.chicagotribune.com/1991-03-03/features/9101200116_1_haakon-haakonsen-director-nils-gauphakon.

284 "Skábmagovat 2015", Skábmagovat Film Festival, Microsoft Word document courtesy of Kaija Anttonen.

285 "Skábmagovat 2015", Skábmagovat Film Festival, Microsoft Word document courtesy of Kaija Anttonen. 


\begin{tabular}{|c|c|c|c|c|c|c|c|}
\hline $\begin{array}{l}\text { Snolejoni } \\
\mathrm{nna}^{286}\end{array}$ & FI & & Unknown & Sofia Jannok & 2015 & $4 \min$ & $\begin{array}{l}\text { Music } \\
\text { Video }\end{array}$ \\
\hline $\begin{array}{l}\text { Soajjalac } \\
\text { cat }^{287}\end{array}$ & FI & & Unknown & $\begin{array}{l}\text { Ima Aikio- } \\
\text { Arianaick }\end{array}$ & 2013 & $\begin{array}{l}28 \\
\min \end{array}$ & $\begin{array}{l}\text { Docum } \\
\text { entary }\end{array}$ \\
\hline $\begin{array}{l}\text { Social } \\
\text { Animals } \\
288\end{array}$ & RU & & Unknown & Ilya Zheltyakov & 2013 & $\begin{array}{l}16 \\
\min \end{array}$ & $\begin{array}{l}\text { Docum } \\
\text { entary }\end{array}$ \\
\hline $\begin{array}{l}\text { Son of } \\
\text { the } \operatorname{Sun}^{289}\end{array}$ & $\mathrm{SE}$ & & Unknown & Gunilla Bresky & 2017 & $\begin{array}{l}58 \\
\min \end{array}$ & $\begin{array}{l}\text { Docum } \\
\text { entary }\end{array}$ \\
\hline $\begin{array}{l}\text { Sound of } \\
\text { the Sami } \\
\text { Plains }{ }^{290}\end{array}$ & $\mathrm{NO}$ & & Unknown & $\begin{array}{l}\text { Britt Jorunn } \\
\text { Hundsnes }\end{array}$ & 2015 & $\begin{array}{l}58 \\
\min \end{array}$ & $\begin{array}{l}\text { Docum } \\
\text { entary }\end{array}$ \\
\hline $\begin{array}{l}\text { Sparruoa } \\
\text { bban }^{291}\end{array}$ & FI & & Unknown & Suvi West & 2016 & $\begin{array}{l}67 \\
\min \end{array}$ & $\begin{array}{l}\text { Docum } \\
\text { entary }\end{array}$ \\
\hline Spel $^{292}$ & $\mathrm{SE}$ & $\begin{array}{l}\text { Direktörn } \\
\text { och }\end{array}$ & $\begin{array}{l}\text { Private } \\
\text { company }\end{array}$ & Amanda Kernell & 2008 & $\begin{array}{l}13 \\
\min \end{array}$ & Short \\
\hline
\end{tabular}

286 "Skábmagovat 2016: Kaamoksen Kuvia/Reflections of the Endless Night", Inari: Skábmagovat Film Festival, 2016

287 "Dellie Maa Program", Dellie Maa Film Festival, 2014, digital PDF file courtesy of Oskar Östergren.

288 "Skábmagovat 2014", Skábmagovat Film Festival, Microsoft Word document courtesy of Kaija Anttonen.

289 "Son of the Sun", Skábmagovat Film Festival, accessed June 222017, http://skabmagovat.fi/skabmagovat_2014/?page_id=10523.

290 "Sound of the Sámi Plains", Skábmagovat Film Festival, accessed June 222017, http://skabmagovat.fi/skabmagovat_2014/?page_id=8403.

291 "Skábmagovat 2016: Kaamoksen Kuvia/Reflections of the Endless Night", Inari: Skábmagovat Film Festival, 2016.

292 "Amanda Kernell", Swedish Film Institute Database, accessed May 9 2017, http://www.sfi.se/en-GB/Swedishfilm-

database/Item/?type=PERSON\&itemid=325817\&iv=MOVIE\&ref=\%2ftemplates $\% 2 \mathrm{fSwedishFilmSearchResult.asp}$ $\mathrm{x} \% 3$ fid $\% 3 \mathrm{~d} 1225 \% 26$ epslanguage $\% 3 \mathrm{den}-$

GB $\% 26$ searchword\%3damanda+kernell\%26type $\% 3 \mathrm{dPerson} \% 26 \mathrm{match} \% 3 \mathrm{dBegin} \% 26 \mathrm{page} \% 3 \mathrm{~d} 1 \% 26 \mathrm{prom} \% 3 \mathrm{dFalse}$. 


\begin{tabular}{|c|c|c|c|c|c|c|c|}
\hline & & $\begin{array}{l}\text { Fabrikörn } \\
\text { Handelsbola } \\
\text { g }\end{array}$ & & & & & \\
\hline $\begin{array}{l}\text { Spin and } \\
\text { Feast, } \\
\text { Ferret }\end{array}$ & FI & $\begin{array}{l}\text { Ima filbma- } \\
\text { ja } \\
\text { sátneduodji } \\
\text { \& Inarin } \\
\text { elokuvateoll } \\
\text { isuus }\end{array}$ & $\begin{array}{l}\text { Mixed } \\
\text { (private } \\
\text { company, } \\
\text { non-profit) }\end{array}$ & $\begin{array}{l}\text { Ima Aikio- } \\
\text { Arianaick }\end{array}$ & 2014 & $3 \mathrm{~min}$ & Short \\
\hline $\begin{array}{l}\text { State of } \\
\text { the } \\
\text { Nations } \\
(250 \\
\text { Years } \\
\text { Honour } \\
\text { Your } \\
\text { Words })^{294}\end{array}$ & $\mathrm{CA}$ & $\begin{array}{l}\text { Elle-Maija } \\
\text { Tailfeathers }\end{array}$ & Individual & $\begin{array}{l}\text { Elle-Maija } \\
\text { Tailfeathers }\end{array}$ & 2013 & $6 \mathrm{~min}$ & $\begin{array}{l}\text { Docum } \\
\text { entary }\end{array}$ \\
\hline $\begin{array}{l}\text { Stoerre } \\
\text { Vaerie }^{295}\end{array}$ & & $\begin{array}{l}\text { Oskar } \\
\text { Östergren, } \\
\text { Bautafilm }\end{array}$ & $\begin{array}{l}\text { Mixed } \\
\text { (individual, } \\
\text { private }\end{array}$ & Amanda Kernell & 2015 & $\begin{array}{l}15 \\
\min \end{array}$ & Short \\
\hline
\end{tabular}

293 "Skábmagovat 2015", Skábmagovat Film Festival, Microsoft Word document courtesy of Kaija Anttonen.

294 "Film and Video Works", Elle-Maija Tailfeathers.com, accessed May 9 2017, http://elle-maijatailfeathers.com/?page_id=311

295 "7 Sámi Films", International Sámi Film Institute, accessed May 92017, http://www.isfi.no/eng/films/7Sámistories. 


\begin{tabular}{|c|c|c|c|c|c|c|c|}
\hline & & & company) & & & & \\
\hline $\begin{array}{l}\text { Tawhaow } \\
\text { haoo }^{296}\end{array}$ & $\mathrm{NZ}$ & & Unknown & $\begin{array}{l}\text { Himiona Grace, } \\
\text { Blackhorse Lowe, } \\
\text { Sunna } \\
\text { Nousuniemi }\end{array}$ & 2016 & $6 \min$ & Short \\
\hline $\begin{array}{l}\text { The } \\
\text { Borderlin } \\
\text { es of } \\
\text { Memory } \\
297\end{array}$ & FI & & Unknown & Tiina Meling & 2013 & $\begin{array}{l}20 \\
\min \end{array}$ & $\begin{array}{l}\text { Docum } \\
\text { entary }\end{array}$ \\
\hline $\begin{array}{l}\text { The } \\
\text { Fire }^{298}\end{array}$ & SE & & Unknown & Liselotte Wajstedt & 2017 & $\begin{array}{l}9 \mathrm{~min} \\
50 \mathrm{sec}\end{array}$ & Short \\
\hline $\begin{array}{l}\text { The } \\
\text { Kautokei } \\
\text { no } \\
\text { Confront } \\
\text { ation I } \\
\text { and } \mathrm{II}^{299}\end{array}$ & $\mathrm{NO}$ & NRK & State Media & Arne O. Holm & 2006 & $\begin{array}{l}2 \times 29 \\
\min \end{array}$ & $\begin{array}{l}\text { Docum } \\
\text { entary }\end{array}$ \\
\hline $\begin{array}{l}\text { The King } \\
\text { Crab - }\end{array}$ & FI & $\begin{array}{l}\text { Unilumi } \\
\text { Oy/Uhak }\end{array}$ & $\begin{array}{l}\text { Private } \\
\text { Company, }\end{array}$ & Helena Sahavirta & 2003 & $\begin{array}{l}30 \\
\min \end{array}$ & $\begin{array}{l}\text { Docum } \\
\text { entary }\end{array}$ \\
\hline
\end{tabular}

296 "Tawhaowao", Skábmagovat Film Festival, accessed June 22 2017, http://skabmagovat.fi/skabmagovat_2014/?page_id=8649.

297 "Skábmagovat 2014", Skábmagovat Film Festival, Microsoft Word document courtesy of Kaija Anttonen.

298 "CV", Liselotte Wajstedt, accessed May 9 2017, http://www.liselottewajstedt.com/bio.

299 "Skábmagovat 2007", Skábmagovat Film Festival, accessed May 9 2017, http://www.Skábmagovat.fi/Skábma_vanhat/Skábma_2007/800en.html. 


\begin{tabular}{|l|l|l|l|l|l|l|l|}
\hline $\begin{array}{l}\text { Threat or } \\
\text { Treasure } \\
\text { of the }\end{array}$ & & iisi Koivisto & unknown & & & & \\
Barents \\
Sea
\end{tabular}

300 "Skábmagovat 2004", Skábmagovat Film Festival, accessed May 9 2017, http://www.Skábmagovat.fi/Skábma_vanhat/Skábma_2004/800en.html.

301 "Skábmagovat 2002", Skábmagovat Film Festival, accessed June 22 2017, http://www.skabmagovat.fi/skabma_vanhat/skabma_2002/800en.html.

302 "The Last King", Norwegian Film Institute, accessed June 22 2017, http://www.nfi.no/english/119346/birkebeinerne.

303"The Light", Skabmagovat Film Festival, accessed June 22 2017, http://skabmagovat.fi/skabmagovat_2014/?page_id=8399 .

304 "CV", Liselotte Wajstedt, accessed May 9 2017, http://www.liselottewajstedt.com/bio. 


\begin{tabular}{|c|c|c|c|c|c|c|c|}
\hline $\begin{array}{l}\text { Thing to } \\
\text { Do }^{305}\end{array}$ & & Tailfeathers & & Tailfeathers & & $16 \mathrm{sec}$ & \\
\hline $\begin{array}{l}\text { The Sámi } \\
\text { and Her } \\
\text { Body }^{306}\end{array}$ & SE & & Unknown & Liselotte Wajstedt & 2007 & $\begin{array}{l}4 \mathrm{~min} \\
52 \mathrm{sec}\end{array}$ & Short \\
\hline $\begin{array}{l}\text { The } \\
\text { Sun } 307\end{array}$ & FI & Stadia & Unknown & Sampo Karvo & 2002 & $\begin{array}{l}10 \\
\min \end{array}$ & Short \\
\hline $\begin{array}{l}\text { This is } \\
\text { My } \\
\text { Land }^{308}\end{array}$ & SE & & Unknown & Sofia Jannok & 2016 & $6 \min$ & $\begin{array}{l}\text { Music } \\
\text { Video }\end{array}$ \\
\hline $\begin{array}{l}\text { To Kill a } \\
\text { Reindeer } \\
309\end{array}$ & FI & Safi Oy & $\begin{array}{l}\text { Private } \\
\text { Company }\end{array}$ & $\begin{array}{l}\text { Paul-Anders } \\
\text { Simma }\end{array}$ & 2012 & $\begin{array}{l}59 \\
\min \end{array}$ & $\begin{array}{l}\text { Docum } \\
\text { entary }\end{array}$ \\
\hline $\begin{array}{l}\text { Trambo } \\
310\end{array}$ & FI & & Unknown & Marja Helander & 2013 & $4 \mathrm{~min}$ & Short \\
\hline $\begin{array}{l}\text { Triffon } \\
\text { from }\end{array}$ & & $\begin{array}{l}\text { Yleisradio } \\
\text { TV 2, SVT }\end{array}$ & State Media & $\begin{array}{l}\text { Elli Rantala, Sulo } \\
\text { Aikio }\end{array}$ & 1983 & $\begin{array}{l}20 \\
\min \end{array}$ & $\begin{array}{l}\text { Docum } \\
\text { entary }\end{array}$ \\
\hline
\end{tabular}

305 "Film and Video Works", Elle-Maija Tailfeathers.com, accessed May 9 2017, http://elle-maijatailfeathers.com/?page_id=311.

306 "CV", Liselotte Wajstedt, accessed May 9 2017, http://www.liselottewajstedt.com/bio.

307 "Skábmagovat 2004", Skábmagovat Film Festival, accessed May 92017, http://www.Skábmagovat.fi/Skábma_vanhat/Skábma_2004/800en.html.

308 "This is My Land", Skábmagovat Film Festival, accessed June 22 2017, http://skabmagovat.fi/skabmagovat_2014/?page_id=8362.

309 "To Kill a Reindeer", DokWeb, accessed May 9 2017, http://archive.dokweb.net/en/documentary-network/easteuropean-docs/-to-kill-a-reindeer-6549.

310 "Skábmagovat 2014", Skábmagovat Film Festival, Microsoft Word document courtesy of Kaija Anttonen. 


\begin{tabular}{|c|c|c|c|c|c|c|c|}
\hline $\begin{array}{l}\text { Petcheng } \\
\mathrm{a}^{311}\end{array}$ & & & & & & & \\
\hline $\begin{array}{l}\text { Troll } \\
\text { Hideawa } \\
\mathrm{y}^{312}\end{array}$ & FI & $\begin{array}{l}\text { Ima filbma- } \\
\text { ja } \\
\text { sátneduodji } \\
\text { \& Inarin } \\
\text { elokuvateoll } \\
\text { isuus }\end{array}$ & $\begin{array}{l}\text { Mixed } \\
\text { (private } \\
\text { company, } \\
\text { non-profit) }\end{array}$ & $\begin{array}{l}\text { Ima Aikio- } \\
\text { Arianaick }\end{array}$ & 2014 & $3 \mathrm{~min}$ & Short \\
\hline $\begin{array}{l}\text { Turistern } \\
\mathrm{e}^{313}\end{array}$ & $\mathrm{NO}$ & & Unknown & Mai-Lis Eira & 2014 & $9 \min$ & $\begin{array}{l}\text { Docum } \\
\text { entary }\end{array}$ \\
\hline $\begin{array}{l}\text { Våra } \\
\text { Discon } \\
314\end{array}$ & SE & $\begin{array}{l}\text { Direktörn } \\
\text { och } \\
\text { fabrikörn } \\
\text { handelsbola } \\
\text { g }\end{array}$ & $\begin{array}{l}\text { Private } \\
\text { Company }\end{array}$ & Amanda Kernell & 2007 & $8 \mathrm{~min}$ & Short \\
\hline Våren 315 & $\mathrm{SE}$ & & Unknown & Patrick Muskos & 2003 & $3 \mathrm{~min}$ & Short \\
\hline $\begin{array}{l}\text { Vidas } \\
\text { Voktere }\end{array}$ & $\mathrm{NO}$ & NRK Sapmi & State Media & Roger Mandal & 2014 & $\begin{array}{l}30 \\
\min \end{array}$ & $\begin{array}{l}\text { Docum } \\
\text { entary }\end{array}$ \\
\hline
\end{tabular}

311 "Skábmagovat 2003," Skábmagovat Film Festival, accessed May 9 2017, http://www.Skábmagovat.fi/Skábma_vanhat/Skábma_2003/800en.html.

312 "Skábmagovat 2015", Skábmagovat Film Festival, Microsoft Word document courtesy of Kaija Anttonen.

313 "Skábmagovat 2016: Kaamoksen Kuvia/Reflections of the Endless Night", Inari: Skábmagovat Film Festival, 2016.

314 "Amanda Kernell", Swedish Film Institute Database, accessed May 9 2017, http://www.sfi.se/en-GB/Swedishfilm-

database/Item/?type=PERSON\&itemid=325817\&iv=MOVIE\&ref=\%2ftemplates $\% 2 \mathrm{fS}$ wedishFilmSearchResult.asp $\mathrm{x} \% 3$ fid $\% 3 \mathrm{~d} 1225 \% 26$ epslanguage $\% 3 \mathrm{den}-$

GB\%26searchword\%3damanda+kernell\%26type \%3dPerson \%26match\%3dBegin\%26page\%3d1\%26prom\%3dFalse.

315 "Skábmagovat 2014", Skábmagovat Film Festival, Microsoft Word document courtesy of Kaija Anttonen. 


\begin{tabular}{|c|c|c|c|c|c|c|c|}
\hline 316 & & & & & & & \\
\hline $\begin{array}{l}\text { Vihtta } \\
\text { Vahkku } \\
\text { Vieljain } \\
317\end{array}$ & $\mathrm{NO}$ & & Unknown & $\begin{array}{l}\text { Bieret Ravdna } \\
\text { Rognerud Eira }\end{array}$ & 2016 & $\begin{array}{l}17 \\
\min \end{array}$ & $\begin{array}{l}\text { Docum } \\
\text { entary }\end{array}$ \\
\hline Viikate $^{318}$ & FI & Sogsakk & Educational & Jonne Jarvinen & 2014 & $\begin{array}{l}12 \\
\min \end{array}$ & Short \\
\hline $\begin{array}{l}\text { Vilda } \\
\text { Duottar } \\
319\end{array}$ & $\mathrm{NO}$ & ISFI & Non-profit & $\begin{array}{l}\text { Johan Ante Utsi, } \\
\text { Inga Marie Risvik, } \\
\text { Elle Marja P. Eira, } \\
\text { Yvonne } \\
\text { Thommassen, } \\
\text { Anne Merete A. } \\
\text { Gaup, Hakon Isak } \\
\text { Vars }\end{array}$ & 2009 & $6 \mathrm{~min}$ & Short \\
\hline $\begin{array}{l}\text { Virtauksi } \\
\mathrm{a}^{320}\end{array}$ & FI & Sogsakk & Educational & Tapio Seppala & 2014 & $\begin{array}{l}13 \\
\min \end{array}$ & Short \\
\hline $\begin{array}{l}\text { Vuoinnal } \\
\text { as }\end{array}$ & $\mathrm{NO}$ & & Unknown & Gjert Rognli & 2009 & $5 \mathrm{~min}$ & Short \\
\hline
\end{tabular}

316 "Viddas Voktere", Skábmagovat Film Festival, accessed June 222017, http://skabmagovat.fi/skabmagovat_2014/?page_id=8679.

317 "Vihtta Vahhku Vieljain", Skábmagovat Film Festival, accessed June 22 2017, http://skabmagovat.fi/skabmagovat_2014/?page_id=8434.

318 "Skábmagovat 2015", Skábmagovat Film Festival, Microsoft Word document courtesy of Kaija Anttonen.

319 "Short Films Funded by the International Sámi Film Centre", International Sámi Film Institute, accessed May 9 2017, http://www.isfi.no/eng/films/short.

320 "Skábmagovat 2015", Skábmagovat Film Festival, Microsoft Word document courtesy of Kaija Anttonen. 


\begin{tabular}{|c|c|c|c|c|c|c|c|}
\hline cumma $^{321}$ & & & & & & & \\
\hline $\begin{array}{l}\text { Vuosttas } \\
\text { lavkkit }^{322}\end{array}$ & $\mathrm{NO}$ & NRK Sápmi & State Media & $\begin{array}{l}\text { Ole Mahtte H. } \\
\text { Gaup }\end{array}$ & 2014 & $\begin{array}{l}39 \\
\min \end{array}$ & $\begin{array}{l}\text { Docum } \\
\text { entary }\end{array}$ \\
\hline $\begin{array}{l}\text { We are } \\
\text { Still } \\
\text { Here }^{323}\end{array}$ & SE & & Unknown & $\begin{array}{l}\text { Sofia Jannok, } \\
\text { Matti Aikio }\end{array}$ & 2016 & $5 \min$ & $\begin{array}{l}\text { Music } \\
\text { Video }\end{array}$ \\
\hline $\begin{array}{l}\text { Where } \\
\text { the } \mathrm{F}^{*} \mathrm{ck} \\
\text { is } \\
\text { Vuotso? } \\
324\end{array}$ & FI & $\begin{array}{l}\text { Tuupa } \\
\text { Records }\end{array}$ & Music Label & Jussi Isokoski & 2014 & $3 \min$ & $\begin{array}{l}\text { Music } \\
\text { Video }\end{array}$ \\
\hline $\begin{array}{l}\text { Who is } \\
\text { Left on } \\
\text { the } \\
\text { Shore? }\end{array}$ & $\mathrm{NO}$ & Hommit AS & $\begin{array}{l}\text { Private } \\
\text { Company }\end{array}$ & Niillas A. Somby & 2003 & $\begin{array}{l}38 \\
\min \end{array}$ & $\begin{array}{l}\text { Docum } \\
\text { entary }\end{array}$ \\
\hline $\begin{array}{l}\text { Wimme } \\
326\end{array}$ & FI & Unilumi & $\begin{array}{l}\text { Private } \\
\text { Company }\end{array}$ & $\begin{array}{l}\text { Juha-lisakki } \\
\text { Koivisto }\end{array}$ & 2008 & $\begin{array}{l}30 \\
\min \end{array}$ & $\begin{array}{l}\text { Docum } \\
\text { entary }\end{array}$ \\
\hline $\begin{array}{l}\text { Ylva: } \\
\text { Lipn }^{327}\end{array}$ & Sápmi & & Unknown & Suohpanterror & 2016 & $7 \mathrm{~min}$ & $\begin{array}{l}\text { Music } \\
\text { Video }\end{array}$ \\
\hline
\end{tabular}

321 "Skábmagovat 2010", Skábmagovat Film Festvial, accessed May 9 2017, http://www.Skábmagovat.fi/Skábma_vanhat/Skábma_2010/800en.html.

${ }^{322}$ Skábmagovat 2015", Skábmagovat Film Festival, Microsoft Word document courtesy of Kaija Anttonen.

323 "We are Still Here", ImagineNative, accessed May 9 2017, http://www.imaginenative.org/we-are-still-here.

324 "Skábmagovat 2015", Skábmagovat Film Festival, Microsoft Word document courtesy of Kaija Anttonen.

325 "Skábmagobat 2004", Skábmagovat Film Festival, accessed May 9 2017, http://www.Skábmagovat.fi/Skábma_vanhat/Skábma_2004/800en.html.

326 "Skábmagovat 2009", Skábmagovat Film Festival, accessed May 9 2017, http://www.Skábmagovat.fi/Skábma_vanhat/Skábma_2009/800en.html. 


\begin{tabular}{|l|l|l|l|l|l|l|l|}
\hline Yoik & SE & & Unknown & Liselotte Wajstedt & & 3 min & $\begin{array}{l}\text { Docum } \\
\text { entary }\end{array}$ \\
\hline $\begin{array}{l}\text { Yoiking } \\
\text { Away }^{329}\end{array}$ & FI & & Unknown & Julani Valasvaara & 1971 & 30 & Docum \\
\hline
\end{tabular}

327 "Ylva: Lipn", Skábmagovat Film Festival, accessed June 22 2017, http://skabmagovat.fi/skabmagovat_2014/?page_id=8373.

328"Skábmagovat 2008", Skábmagovat Film Festival, accessed May 92017 , http://www.Skábmagovat.fi/Skábma_vanhat/Skábma_2008/ohjelma_en/ohjelmainfo_su.htm.

329 "Skábmagovat 2013", Skábmagobat Film Festival, accessed May 92017 , http://www.Skábmagovat.fi/Skábma_vanhat/Skábma_2013/800en.html. 


\section{Appendix D: Sámi Films by the Numbers}

Number of Films by Type of Producer in Each Country (Database)

\begin{tabular}{|c|c|c|c|c|c|c|c|c|c|}
\hline & $\begin{array}{l}\text { Education } \\
\text { al }\end{array}$ & $\begin{array}{l}\text { Individu } \\
\text { al }\end{array}$ & $\begin{array}{l}\text { State } \\
\text { Med } \\
\text { ia }\end{array}$ & $\begin{array}{l}\text { Compa } \\
\text { ny }\end{array}$ & $\begin{array}{l}\text { Non-Profit } \\
\text { or } \\
\text { Collective }\end{array}$ & $\begin{array}{l}\text { Mixe } \\
\text { d }\end{array}$ & $\begin{array}{l}\text { Musi } \\
\text { c } \\
\text { Labe } \\
1\end{array}$ & $\begin{array}{l}\text { Unknow } \\
\text { n or not } \\
\text { specifie } \\
\text { d }\end{array}$ & $\begin{array}{l}\text { Tota } \\
1\end{array}$ \\
\hline Sweden & 3 & 9 & 5 & 14 & 1 & 5 & 0 & 2 & 39 \\
\hline Norway & 13 & 11 & 26 & 27 & 3 & 17 & 1 & 15 & 113 \\
\hline Finland & 96 & 3 & 7 & 14 & 13 & 4 & 2 & 5 & 144 \\
\hline Russia & 0 & 0 & 0 & 0 & 0 & 1 & 0 & 0 & 1 \\
\hline $\begin{array}{l}\text { Sápmi } \\
\text { Joint } \\
\text { incl. } \\
\text { Sweden }\end{array}$ & 0 & 0 & 2 & 3 & 0 & 3 & 0 & 0 & 8 \\
\hline $\begin{array}{l}\text { Sápmi } \\
\text { joint not } \\
\text { incl. } \\
\text { Sweden }\end{array}$ & 0 & 0 & 2 & 2 & 0 & 0 & 0 & 0 & 4 \\
\hline $\begin{array}{l}\text { Sápmi/N } \\
\text { on- } \\
\text { Sápmi }\end{array}$ & 1 & 0 & 0 & 2 & 0 & 0 & 0 & 1 & 4 \\
\hline
\end{tabular}




\begin{tabular}{|c|c|c|c|c|c|c|c|c|c|}
\hline $\begin{array}{l}\text { joint } \\
\text { inclu. } \\
\text { Sweden }\end{array}$ & & & & & & & & & \\
\hline $\begin{array}{l}\text { Sápmi/N } \\
\text { on- } \\
\text { Sápmi } \\
\text { joint not } \\
\text { incl. } \\
\text { Sweden }\end{array}$ & 1 & 0 & 0 & 1 & 0 & 0 & 0 & 1 & 3 \\
\hline $\begin{array}{l}\text { Non- } \\
\text { Sápmi }\end{array}$ & 0 & 1 & 0 & 0 & 1 & 0 & 0 & 0 & 2 \\
\hline $\begin{array}{l}\text { Country } \\
\text { not } \\
\text { specified }\end{array}$ & 1 & 0 & 2 & 1 & 0 & 0 & 0 & 1 & 5 \\
\hline Total & 115 & 24 & 44 & 64 & 18 & 30 & 3 & 25 & 323 \\
\hline
\end{tabular}


Number of Films by Type of Producer in Each Country (Outside Database)

\begin{tabular}{|l|l|l|l|l|l|l|l|l|l|}
\hline & Education & Individu & State & Compan & Non- & Musi & Mixe & Unknow & Tota \\
& al & Medi & y & Profit or & c & d & n & 1 \\
Collecti & Labe & & & \\
\hline Sweden & 0 & 0 & 1 & 10 & 0 & 0 & 3 & 27 & 41 \\
\hline Norway & 2 & 2 & 13 & 10 & 1 & 0 & 2 & 19 & 49 \\
\hline Finland & 15 & 0 & 1 & 7 & 0 & 2 & 0 & 28 & 53 \\
\hline Russia & 0 & 0 & 0 & 0 & 0 & 0 & 0 & 2 & 2 \\
\hline Sápmi & 0 & 0 & 0 & 0 & 0 & 0 & 0 & 1 & 1 \\
(otherwise \\
unspecifie \\
d)
\end{tabular}




\begin{tabular}{|c|c|c|c|c|c|c|c|c|c|}
\hline $\begin{array}{l}\text { joint inclu. } \\
\text { Sweden }\end{array}$ & & & & & & & & & \\
\hline $\begin{array}{l}\text { Sápmi/No } \\
\text { n-Sápmi } \\
\text { joint not } \\
\text { incl. } \\
\text { Sweden }\end{array}$ & 0 & 0 & 0 & 1 & 0 & 0 & 0 & 0 & 1 \\
\hline $\begin{array}{l}\text { Non- } \\
\text { Sápmi }\end{array}$ & 0 & 6 & 1 & 0 & 0 & 0 & 0 & 3 & 10 \\
\hline $\begin{array}{l}\text { Country } \\
\text { Not } \\
\text { Specified }\end{array}$ & 0 & 0 & 1 & 4 & 0 & 0 & 6 & 4 & 15 \\
\hline Total & 17 & 8 & 17 & 34 & 1 & 2 & 11 & 85 & 175 \\
\hline
\end{tabular}

Sámi Film Database by Country

\begin{tabular}{|l|l|l|}
\hline Country & Number & SE included? (Joint) \\
\hline Norway & 113 & N/A \\
\hline Sweden & 38 & N/A \\
\hline Finland & 144 & N/A \\
\hline Russia & 1 & N/A \\
\hline Joint (Sápmi only) & 13 & 9 \\
\hline
\end{tabular}




\begin{tabular}{|l|l|l|}
\hline Joint (Sápmi/non-Sápmi) & 6 & 3 \\
\hline Exclusively non-Sápmi & 2 & N/A \\
\hline Country not specified & 6 & N/A \\
\hline
\end{tabular}

Sámi Film Database by Genre

\begin{tabular}{|l|l|}
\hline Genre $^{330}$ & Number \\
\hline Children's Film & 7 \\
\hline Documentary & 100 \\
\hline Feature & 12 \\
\hline Short & 194 \\
\hline TV Production & 9 \\
\hline
\end{tabular}

Films Outside the Database by Country

\begin{tabular}{|l|l|l|}
\hline Country & Number & SE Included? \\
\hline Sweden & 41 & N/A \\
\hline Norway & 49 & N/A \\
\hline Finland & 53 & N/A \\
\hline Russia & 2 & N/A \\
\hline $\begin{array}{l}\text { Sápmi (not } \\
\text { otherwise }\end{array}$ & 1 & N/A \\
\hline
\end{tabular}

${ }^{330}$ Taken from the descriptions in the Sámi Film Database - see Appendix C. 


\begin{tabular}{|l|l|l|}
\hline specified) & & \\
\hline Other & 10 & N/A \\
\hline Joint (Sápmi) & 2 & 1 \\
\hline $\begin{array}{l}\text { Joint } \\
\text { (Sápmi/Non- } \\
\text { Sápmi) }\end{array}$ & 2 & 1 \\
\hline $\begin{array}{l}\text { Country not } \\
\text { specified }\end{array}$ & 15 & N/A \\
\hline
\end{tabular}

Films Outside the Database by Genre

\begin{tabular}{|l|l|}
\hline Genre $^{331}$ & Number \\
\hline Documentary & 50 \\
\hline Feature & 8 \\
\hline Remix & 1 \\
\hline Experimental & 3 \\
\hline Short & 71 \\
\hline TV Production & 7 \\
\hline Music Video & 20 \\
\hline Genre not specified & 15 \\
\hline
\end{tabular}

${ }^{331}$ Taken from the descriptions of the Skábmagovat programme (or other film festivals), the filmmakers' own writing, or other film databases, depending on the source. 


\section{Bibliography}

Sami Films and their Content

Christensen, Cato. "Reclaiming the Past: On the History-Making Significance of the Sámi Film The Kautokeino Rebellion." Acta Borealia: A Nordic Journal of Circumpolar Societies 29, no 1 (2012): 56-76.

Downing, John and Charles Husband. Representing 'Race': Racisms, Ethnicities and Media. London: Sage Publications Ltd., 2005.

Iversen, Gunnar. "Learning from Genre: Genre Cycles in Modern Norwegian Cinema". Transnational Cinema in a Global North: Nordic Cinema in Transition, ed. Andrew K. Nestingten and Trevor Glen Elkington. Detroit: Wayne State University Press, 2005. 261 278.

Lehtola, Veli-Pekka. The Sámi People: Traditions in Transitions. Fairbanks: University of Alaska Press, 2005.

Pearson, Wendy Gay. "Once Upon a Time in a Land Far, Far Away". Reverse Shots: Indigenous Film and Media in an International Context. Ed. Wendy Gay Pearson and Susan Gabe. Waterloo: Wilfrid Laurier University Press, 2015. 147

Pietikainen, Sari. "'To Breathe Two Airs': Empowering Indigenous Sámi Media." in Global indigenous media: Cultures, Poetics, and Politics, ed. P Wilson \& M Stewart. Durham: Duke University Press, 2008. 197-213.

Ramnarine, Tina K. "Sonic Images of the Sacred In Sámi Cinema." Interventions 15, no. 2 (June 2013): 239-254. 
Skardhamar, Anne Yr. "Changes in Film Representations of Sámi Culture and Identity." Nordlitarbeidtstidsskrift i literatur 12, no. 2 (2008): 293-304.

"Sámi Film Database". Skábmagovat.fi. Accessed April 3, 2016. http://www.Skábmagovat.fi/Sámifilm/index.php?lang=en

\section{Sami Political Status and History}

Alia, Valerie. The New Media Nation: Indigenous Peoples and Global Communication. New York: Berghahn Books, 2010.

Allard, Christina and Susann Funderud Skogvang. Indigenous Rights in Scandinavia: Autonomous Sámi Law. New York: Routledge, 2016.

Anaya, James. "The Situation of the Sámi People in the Sápmi Region of Norway, Sweden and Finland." James Anaya: Former United Nations Special Rapporteur On the Rights of Indigenous Peoples. Last Modified June 6, 2011. http://unsr.jamesanaya.org/country reports/the-situation-of-the-Sámi-people-in-the-Sápmi-region-of-norway-sweden-and finland-2011

Markelin, Lia and Charles Husband. "Contemporary Dynamics of Sámi Media in the Nordic States." Media International Australia: Incorporating Culture and Policy 149, Indigenous Media Practice (2013): 70-81.

Markelin, Lia and Charles Husband. "The Sámi Media, State Broadcasting and Transnational Indigeneity." In Transnational Lives and the Media: Re-Imagining Diaspora, ed. Olga G. 
Bailey, Myria Georgiou and Ramaswami Harindranath. London: Palgrave McMillan, 2007. 115-132.

Josefson, Eva. "The Sámi and the National Parliaments: Channels for Political Influence."United Nations Development Programme: New York, 2010. 7-10

Koivurova, Timo. "The Draft for a Nordic Saami Convention". European Yearbook of Minority Rights. Issue 6 (2008): 103. http://www.arcticcentre.org/loader.aspx?id=04e81223028e4315a669-cb8830b22d6b

"Discrimination of the Sámi - the Rights of the Sámi from a Discrimination Perspective". Ombudsmannen mot etnisk diskriminering [Ombudsman against ethnic discrimination]. Stockholm: Lenanders Grafiska AB, 2008

Reed-Bowers, India. "Preparatory Report from the Sámi Parliament in Sweden/Sámediggi/Sámedigge/Saemiedigkie/Sametinget for the United Nations Special Rapporteur on the Rights of Indigenous Peoples, Ms. Victoria Tauli-Corpuz, prior to her 2015 August visit to Sápmi and Sweden" Samediggi, August 2015.

Tauli-Corpuz, Victoria. "Report on the Human Rights Situation of the Sámi People in the Sápmi Region of Norway, Sweden and Finland". United Nations General Assembly Human Rights CouncilThirty- Third Session, Agenda Item 3, 9 August 2016, http://unsr.vtaulicorpuz.org/site/index.php/documents/country-reports/155-report-Sápmi 2016

Vladimirova, Vladislava. "Contested Political Representation of the Sámi in the Kola Peninsula, Northwest Russia." Re: mindings. Co-Constituting Indigenous/Academic/Artistic 
Knowledges, ed. Johan Gärdebro, May-Britt Öhman, Hiroshi Maruyama. Stockholm: Vulkan, 2014. 215-229. 